Portland State University

PDXScholar

$1-1-2011$

\title{
Exploring Traffic Safety and Urban Form in Portland, Oregon
}

Kristie Werner Gladhill

Portland State University

Follow this and additional works at: https://pdxscholar.library.pdx.edu/open_access_etds Let us know how access to this document benefits you.

Recommended Citation

Gladhill, Kristie Werner, "Exploring Traffic Safety and Urban Form in Portland, Oregon" (2011).

Dissertations and Theses. Paper 86.

https://doi.org/10.15760/etd.86

This Thesis is brought to you for free and open access. It has been accepted for inclusion in Dissertations and Theses by an authorized administrator of PDXScholar. Please contact us if we can make this document more accessible: pdxscholar@pdx.edu. 
Exploring Traffic Safety and Urban Form

in Portland, Oregon

by

Kristie Werner Gladhill

A thesis submitted in partial fulfillment of the requirements for the degree of

Master of Science

in

Civil and Environmental Engineering

Thesis Committee:

Christopher M. Monsere, Chair

Kelly Clifton

James Strathman

Portland State University

(C) 2011 


\section{ABSTRACT}

Street layout and design, once established, are then not easily changed. Urban form affects community development, livability, sustainability, and traffic safety. There has been an assumed relationship between urban form and traffic safety that favors designs with less through streets to improve safety. An empirical study to test this assumed relationship was carried out for crash data for Portland, Oregon.

This thesis presents an empirical methodology for analyzing the relationship between urban form and traffic safety utilizing a uniform grid for the spatial unit. Crashes in the Portland, Oregon city limits from 2005-2007 were analyzed and modeled using negative binomial regression to study the effect of urban form and street layout through factors on exposure, connectivity, transit accessibility, demographic factors, and origins and destinations. These relationships were modeled separately by mode: vehicle crashes, pedestrian and bicycle crashes. Models were also developed separately by crash type and by crash injury severity.

The models found that urban form factors of street connectivity and intersection density were not significant at 95\% confidence for vehicle and pedestrian crash rates, nor for different crash severity levels, indicating that high connectivity grid street layout may have comparable safety to loops and 
lollipops, in contrast to results in earlier studies. Elasticity for all models was dominated by VMT increases. Business density, population and transit stops were also significant factors in many models, underlining the importance not only of street layout design, but also planning to direct development to influence where businesses, employment, and housing will grow and handle traffic volumes safely.

Page ii 


\section{DEDICATION}

I thank my parents for valuing education and instilling that drive in me as well, along with perseverance to carry things through. Most of all, though, I thank my husband Richard Gladhill for his un-flagging support and love in this and so many other aspects of our family's life together.

Page iii 


\section{ACKNOWLEDGEMENTS}

I would like to thank my advisor Dr. Chris Monsere and committee members Dr. Kelly Clifton and Dr. James Strathman for their support, guidance, and direction throughout this project. I would also like to thank fellow graduate student Nicole Wheeler for her incredibly generous help with ArcGIS, editing, and reviewing. Thanks also go to Nathan McNeil for sharing geo-coded business data in the Portland area, and April Cutter for help with ArcGIS data.

I could not have done this without the support and help of so many in the Portland State University Department of Civil Engineering, the ITS lab, and OTREC. Having resources for study, ideas, friendships, and work to support me through this time have been a tremendous basis to work from. 
Table of Contents

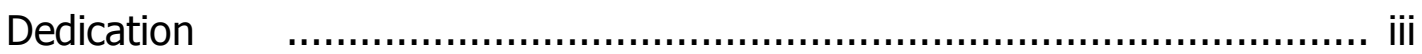

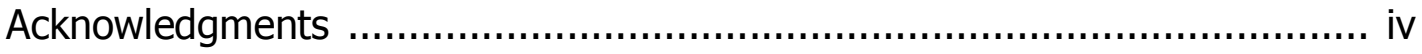

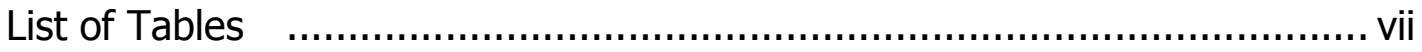

List of Figures $\quad$........................................................................ viii

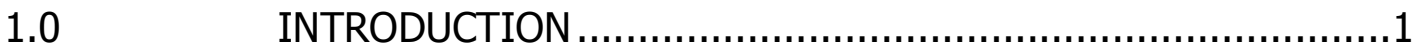

1.1 Problem Statement .............................................................

1.2 Research Objectives and Scope ..............................................

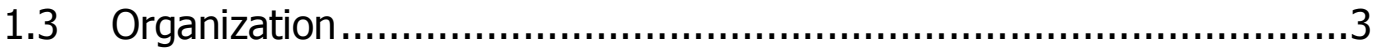

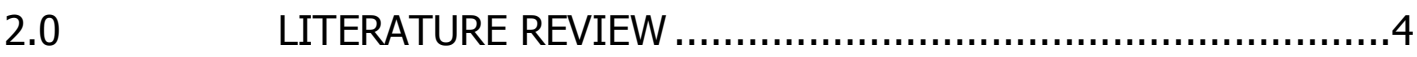

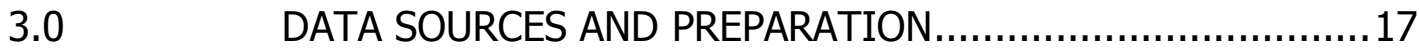

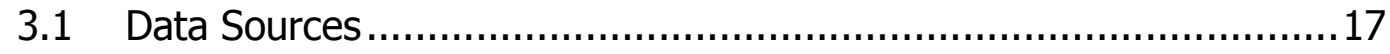

3.1.1 Crash Data ................................................................

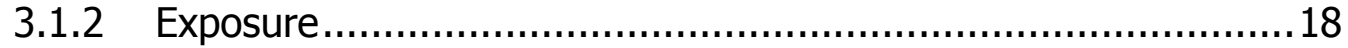

3.1.3 Connectivity ............................................................ 19

3.1.4 Transit Accessibility ..................................................... 19

3.1.5 Demographics, Origins, and Destinations ............................. 20

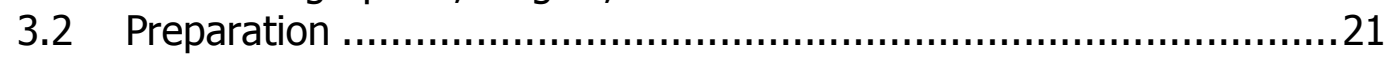

3.2.1 Definition of Spatial Analysis Unit.....................................21

3.2.2 Data Aggregation ............................................................22

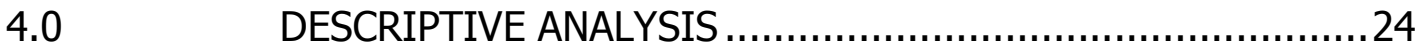

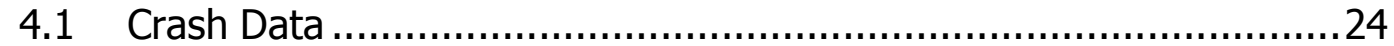

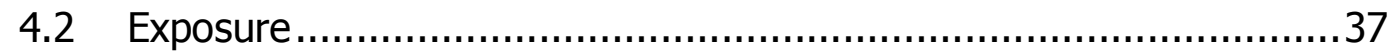

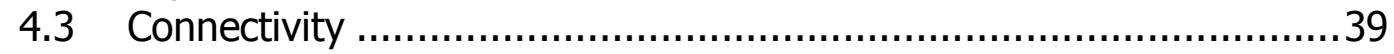

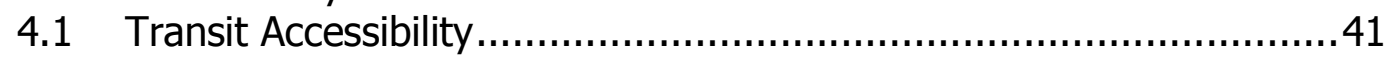

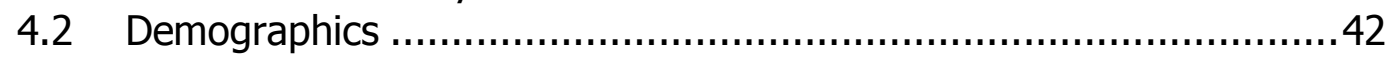

4.3 Origins and Destinations ...................................................... 44

M.0 MODEL DEVELOPMENT AND RESULTS ..........................46

5.1 Development .................................................................. 46

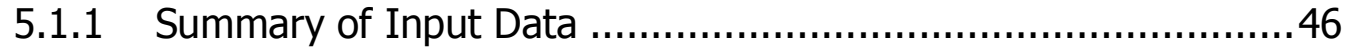

Page $v$ 
5.1.2 Selection of Independent Variables ......................................46

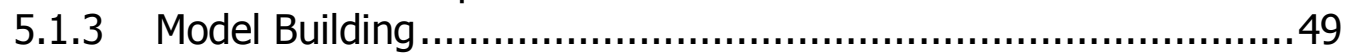

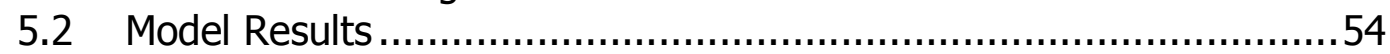

5.2.1 Estimated Coefficients........................................................ 55

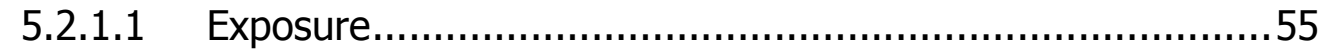

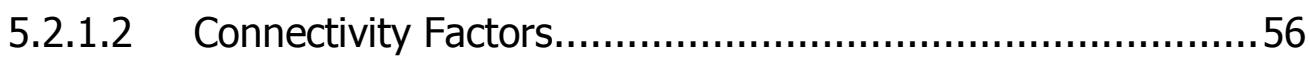

5.2.1.3 Transit accessibility, Demographics, and Origin Destination.57

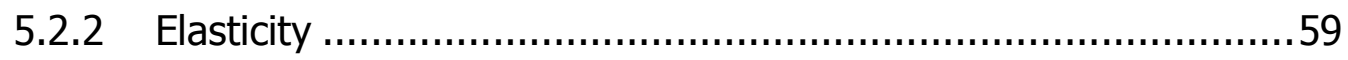

5.2.2.1 By Vehicle, Pedestrian, and Bicycle Models .........................59

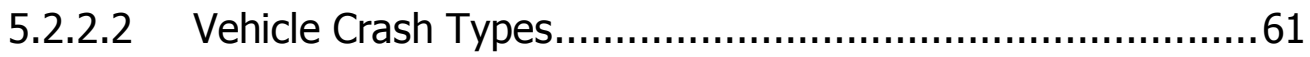

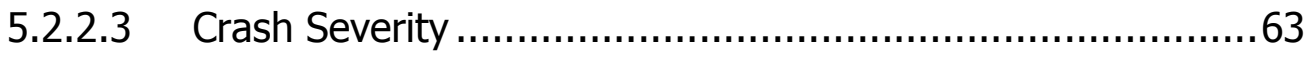

5.2.2.4 Elasticity by Factors ..................................................... 65

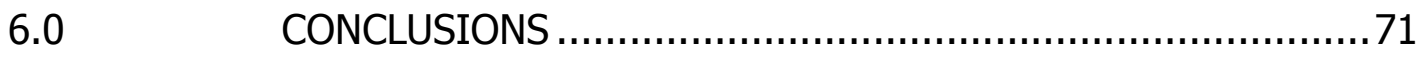

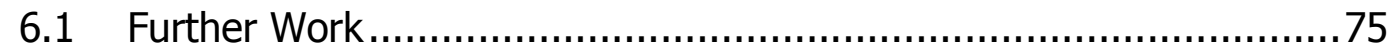

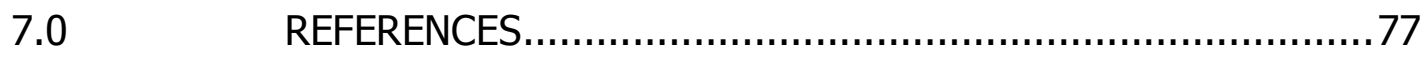

APPENDIX A: R code for the CURE plots ............................................... 79

APPENDIX B: Join point data to spatial area in ArcGIS 9.3.1 .......................86

APPENDIX C: Join Line Data to spatial area in ArcGIS 9.3.1 ......................90

APPENDIX D: Apportion polygon data to a different polygon spatial area

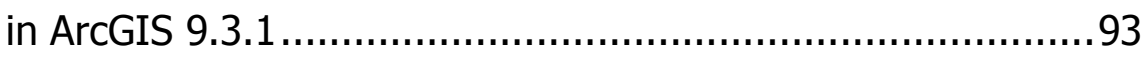

APPENDIX E: Pearson's Correlation on Factors - Figure E-1 Correlation for Exposure Factors ........................................................... 101 


\section{LIST OF TABLES}

Table 1 Summary of Studies Reviewed..................................................14

Table 2 Data aggregated into Uniform Grid Cells for Study ....................23

Table 32005 - 2007 Portland Non-freeway Crashes in Spatial Grid Cells...28

Table $4 \quad 2005$ - 2007 Pedestrian Crashes ...................................................31

Table $5 \quad 2005$ - 2007 Bicycle Crashes ..................................................32

Table 6 Modeling Input Data Summary .............................................48

Table 7 Negative Binomial Modeling Goodness of Fit..............................5 54

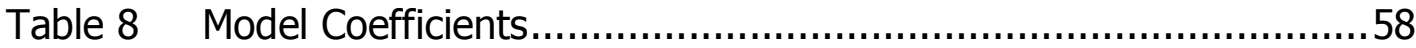




\section{LIST OF FIGURES}

Figure $1 \quad$ Loops and Lollipops ...................................................... 12

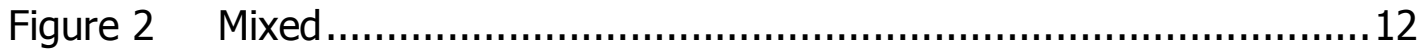

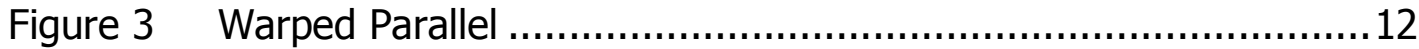

Figure 4 Grid .......................................................................... 12

Figure 5 Spatial Data Grid within the Portland City Limits ......................21

Figure 6 Total Non-freeway Crashes Portland $2005-2007$..................... 25

Figure 7 Histogram of 2006-2007 Crashes in Portland City Limits.............. 25

Figure $8 \quad 2005-2007$ Total Crashes by Crash Type..............................27

Figure 9 Primary Crash Types ......................................................27

Figure 102005 - 2007 Vehicle Crashes by Crash Type, Spatial Maps .........29

Figure 112005 - 2007 Pedestrian Crash Spatial Map .................................33

Figure 122005 - 2007 Bicycle Crash Spatial Map...................................33

Figure 132005 - 2007 Vehicle Crashes .............................................. 34

Figure $14 \quad 2005$ - 2007 Vehicle Crashes, PDO Severity................................ 35

Figure $15 \quad 2005$ - 2007 Vehicle Crashes, FI Severity ..................................35

Figure $16 \quad 2005$ - 2007 Vehicle Crashes, Fatal ........................................36

Figure 172005 - 2007 Vehicle Crashes, Fatal and Injury A Severity............36

Figure 182005 Average of Volume to Capacity Ratios within a Grid Cell.....38

Figure 192005 Average Free Speed .....................................................38

Figure 20 Street Network Value Assignments....................................40

Figure 21 Percentage of Intersections which are Four Leg Intersections .....40

Figure 22 Histogram of Street Network Values..................................... 41

Figure 232007 Transit Stops Spatial Map ...........................................42

Figure 242006 Population ................................................................43

Figure 252006 Dwelling Units Spatial Map ...........................................43

Figure 262005 Employment Spatial Map ...........................................44

Figure 272010 Business Spatial Map ........................................... 45

Figure 28 Cumulative Residual CURE 2 sigma Plots for Comparison.............52

Figure 29 Plots for Negative Binomial Regression Fit ...........................53

Figure 30 Elasticity for Vehicle, Pedestrian, and Bicycle Crashes.................60

Figure 31 Elasticity for Vehicle Crash Types .......................................62

Figure 32 Elasticity for Crash Severity .............................................64

Figure 33 Elasticity for Exposure Factors .......................................67

Figure 34 Elasticity for Street Network Factors..................................68

Figure 35 Elasticity for Connectivity Factors .......................................69

Figure 36 Elasticity for Transit Accessibility, Demographic,

Origin and Destination Factors 


\subsection{INTRODUCTION}

Streets have many functions, both for traffic movement, and as a framework for the neighborhood and local community (1). Street layout and design are typically defined in the planning and design phases for a particular land area. Once infrastructure is established it is then not easily changed. The design and layout of streets affect not only how the local neighborhood and community develop, but also traffic safety. Even though human factors dominate crash causality, it is important to understand secondary effects (2) and interactions with the built environment to aid planning and design of new development. It is important to study the actual safety relative to urban form to check if long held assumptions are valid, so that further development can appropriately consider safety in balance with connectivity and accessibility, along with street design factors such as width, traffic control, and presence of sidewalks. With this understanding, safety can be improved.

Recent studies in San Antonio, Texas (3) and Calgary, Alberta (4), (5) have started to include street layout in the crash analysis, and have shown lower crash rates within the limited through way neighborhoods. 


\subsection{Problem Statement}

Traffic safety has long been a concern in street layout and design. Designers such as Frederick Law Olmsted rejected the grid layout in the 19th century, and started recommending curvilinear streets as part of an idealized suburban lifestyle. Twentieth century designers recommended limited access with limited through way designs for residential areas to reduce through traffic, sacrificing interconnectivity for perceived safety. Although rural road safety benefits from wide lanes and good visibility; urban streets with these features may encourage higher speeds, and thus less safe conditions in an environment that mixes vehicular, pedestrian, and bicycle traffic.

This assumed that a relationship between urban form and traffic safety would favor designs with less through streets to improve safety. Although this premise has been adopted and even codified into national and local standards (1), few studies have looked empirically at differences in traffic safety for different street layout designs. If safe street layout and design features could be identified, designers would be able to recommend urban form(s) that would provide better traffic safety and build safety into the infrastructure, even as connectivity, mobility, and accessibility are also considered. Infrastructure designed for the best safety practices and connectivity for vehicle, pedestrian, 
and bicycle traffic would provide a long term framework for safe and well functioning transportation networks that build and sustain communities.

\subsection{Research Objectives and Scope}

The objective of this research is to empirically quantify the relationship between urban form (defined by exposure, connectivity, transit accessibility, demographics, and origin and destination measures) and traffic safety (defined by reported motor vehicle crashes). This study was undertaken to test whether grid layout, which provides high connectivity and alternate through routes, is any more or less safe than loops and lollipops. The study looked at reported crashes from 2005-2007 within the Portland, Oregon 2007 city limit boundary. A uniform grid was used for spatial analysis to include all crashes without needing to give special consideration to crashes on analysis zone boundaries.

\subsection{Organization}

In the following chapter, this thesis will explore prior work related to traffic safety and urban form in a literature review. Chapter 3 describes the data sources and methodology used for this study. Chapter 4 presents qualitative analysis. Chapter 5 covers quantitative analysis, model building using negative binomial regression models for crashes, model results, and elasticity. Conclusions and recommendations for further work are made in Chapter 6. 


\subsection{LITERATURE REVIEW}

Safety in design of urban streets has been an issue since Roman times (1). Modern studies have looked at not only the street design specifications for right of way and lane layout; but also how the form of the street layout may affect safety along with land use, demographic, and socioeconomic data. This review focuses on published literature that has dealt with empirically modeling urban form and safety.

Perhaps one of the first studies was conducted by Marks who studied five years of crash records on the Los Angeles County street system, encompassing 86 subdivision tracts over 4,320 acres (6). The study was limited to right angle crashes, which were nearly 84 percent of vehicular crashes within subdivisions, and were therefore felt to be representative of most crashes. Major streets bordering the subdivisions were not included in the study, nor was there any adjustment for traffic volume made in the analysis in this study.

Marks found that most crashes occurred at intersections, and some design features increased crash rates. Four leg intersections had much higher crash frequencies than three leg intersections, which have three conflict points compared to sixteen conflict points in a four legged intersection. Features such 
as jogs in alignment, skew, or "Y" shape at intersections were associated with increased crash frequencies. Obstructions to visibility such as bridges or railroad tracks also increased crash frequencies. Intersections spaced too closely were found to be a factor in crash frequencies. Limited access tracts had much lower crash rates than grid layout areas.

Based on these results, Marks recommended elements for safe design which included limited access design; avoidance of continuous through streets; collector streets exiting onto only one major street; preferred use of T-type intersections over four legged intersections; and avoiding multi-leg, Y, skewed, or jog intersections. With guidelines such as these, it was stated that subdividing could be done for safety.

Kim and Levine showed the value of using GIS data for crash analysis studying crashes on Oahu (7). In their analysis, they found that approximately 43 percent of crashes occurred at intersections. Using TIGER (Bureau of Census Topographically Integrated Geographic Encoding and Referencing) data for streets, crashes were assigned to the nearest intersection within 363 block groups for the entire island, including urban areas in Honolulu as well as rural and agricultural uses (8). Crashes were found to be concentrated in built-up, urban areas. Freeways themselves were relatively safe, but freeway ramps and crossroads were particularly dangerous. There was variation in the spatial 
pattern of crashes by time of day, day of week, and different vehicle types. Spatial GIS data for crash analysis was recommended as a tool that could be used to help develop meaningful community safety plans.

Land use activity, pedestrian friendliness, and infrastructure were found to be more effective at reducing road hazard than traffic controls or posted speed limitations in a study by Ossenbruggen et al (9). The study looked at 892 crashes from 87 sites on rural and suburban two lane undivided highways from 1993 to 1997 in Strafford County, New Hampshire. In addition to roadway measurements, qualitative data on land use activity, street life, and vehicle pedestrian interactions were taken at the crash sites. It was found that pedestrian friendly sites were associated with the least hazard, even with high traffic volume. Thus village sites, which were mixed-use areas with sidewalks, were less hazardous than residential or shopping areas without sidewalks. The infrastructure itself and multi-purpose activities seemed to be more effective at warning drivers of the need to proceed cautiously than sites which required traffic control devices to stop or interrupt traffic flow on the main road.

Hadayeghi studied crash frequency and severity in Toronto, Ontario to develop macro-level crash prediction models based on traffic demand, network, economic, and demographic variables (10). Major and minor roadway length in the analysis zone, intersection count, employment, and household population 
increases also increased crash rates. Crash rates decreased with higher posted speed and higher congestion levels. It was suggested that geometry of neighborhood design may also be important, though it had not been available for this study.

Ladron de Guevara (11) showed that planning-level models could be developed that would be useful in MPO (metropolitan planning organization) forecasting. Crashes from 1998 to 1999 in 859 TAZ (transportation analysis zones) in Tucson, AZ were analyzed using negative binomial regression for different levels of crash severity. Demographic and road characteristic variables were studied. Exposure to risk was felt to be better represented in a planning forecasting model by population rather than VMT (vehicle miles traveled) since population would have better future estimates, and was more likely to be available by TAZ than VMT in many jurisdictions. The fatal crash model found both population and population over 17 to be significant factors, along with intersection density. Injury crash parameters also included population density and intersection density, along with employment and miles of arterial and collector roadways.

Al-Masaeid and Suleiman pointed out that reducing the need for travel reduces exposure and traffic, and thus reduces crash risk. They looked at land use, population, VMT, and street network factors in crashes 2001-2002 in the 
Syrian capital of Damascus (12). The land-use factors included percent commercial frontage, green area, industrial, or public buildings. Grid networks had more intersections and higher crash rates than comparable limited access elements. A higher percentage of commercial frontage and public buildings correlated with higher crash rates, suggesting that moving commercial frontage away from major thoroughfares may reduce crash rates. There was multicollinearity found between some of the street network and urban planning variables.

Kim et al in 2006 studied crashes on Oahu further using negative binomial regression analysis to relate crash rates to land use, population and economic activity (13). A grid of uniform sized cells was set up for analysis, rather than TAZ or census block groups. This study showed that grid cell spatial units can be used to statistically model crash rates. Population had a positive relationship to crash frequency, but job count in a particular cell was an even stronger factor to explain crash rates.

Kim et al also looked at using accessibility measures and other demographic and land use attributes to predict crash rates (13). The accessibility measures included road length, bus stops, bus route length, intersections, and dead ends. Uniform 0.1 square mile grid cells were again used for the analysis, with each crash assigned to the nearest intersection. Negative 
binomial regression was not producing a model fit, so logistic regression was used.

Results showed statistical significance for crash rates and vulnerable populations (elderly and children), disability, and job count. None of the demographic factors were significant, but business and land-use factors such as high density residential and military land uses were statistically significant. Bus stops and bus route length correlated with increased pedestrian crashes. Population size was only associated with bicycle crashes. Multicollinear relationships were found with variables such as population size and vulnerable populations (i.e. elderly and children). The researchers concluded that crash predictions could be useful to identify locations needing safety improvement strategies which could be implemented through enforcement, engineering, and education.

Clifton et al studied pedestrian-vehicle crashes in Baltimore, MD to test the hypothesis that the built environment affects injury severity in such crashes (14). In this area, 25 percent of households did not have access to a vehicle though there were numerous public transit options available. This study used more than 4500 pedestrian-vehicle crashes from 2000-2004 that were geocoded to nearest intersection. Analysis (probit model) found that pedestrian connectivity and transit access were the only significant built environment 
variables related to safety. The better these were the less likely severe injuries were to occur. Areas with low connectivity and transit access seemed most in need of safety intervention, since injuries there were more severe. In vehicle pedestrian crashes, children were more likely to sustain injury; the elderly were more likely to be fatalities; and male pedestrians were more likely to sustain injury, especially if substance abuse was involved.

Dumbaugh and Rae questioned whether the design concepts which have become engrained into policies have been empirically tested (3). Using census block groups for San Antonio crashes 2004 to 2006, they analyzed 150,626 reported crashes along with street network and demographic variables. In a methodological assumption, they assigned crashes on peripheral streets to each adjacent spatial unit. This assumption results in "double counting" since a crash would be included in more than one spatial unit. They found that total crash frequency increased with VMT, young or older drivers, and rose fifteen percent with each additional arterial mile. Population density was associated with fewer crashes, attributed to less travel demand when located close to services in densely populated areas. Injury crashes also increased significantly with additional arterials and four leg intersections. Higher income was associated with decreased crash rates. Fatal crashes rose with road and street network elements that increased vehicle speed. 
In conclusion, Dumbaugh and Rae felt that the relationship between community design and traffic safety was important and complex, requiring community-level design solutions beyond simply preventing residential cut through traffic, but which would give attention to how different land uses and street network configurations influence speed and driver behavior. Traffic re-located away from residential areas reduced neighborhood traffic volume. However, arterials designed with wide lanes and long sight distances for higher speeds should have access management, limiting entry and exit points into the high speed traffic. Commercial zones with many entries and exits into the traffic stream should instead be located on lower speed thoroughfares.

Rifaat and Tay looked at injury crash rates (4) and crash severity modeling (5) for Calgary, Alberta. The street network was classified as being one of four patterns: loops and lollipops, mixed, warped parallel, or gridiron, (see Figure 1, Figure 2, Figure 3, and Figure 4). Other factors studied included roadway characteristics, demographics, land use, and socioeconomic factors. Crashes on boundary roadways were not considered due to boundary problems which would have further complicated the model, and since the peripheral traffic was considered to largely be non-local through traffic. Crashes were converted to EPDO (equivalent property damage only) crashes, and different models tried. Limited access patterns were associated with lower crash rates than the gridiron, 
with warped parallel consistently having the lowest crash rates with different models.

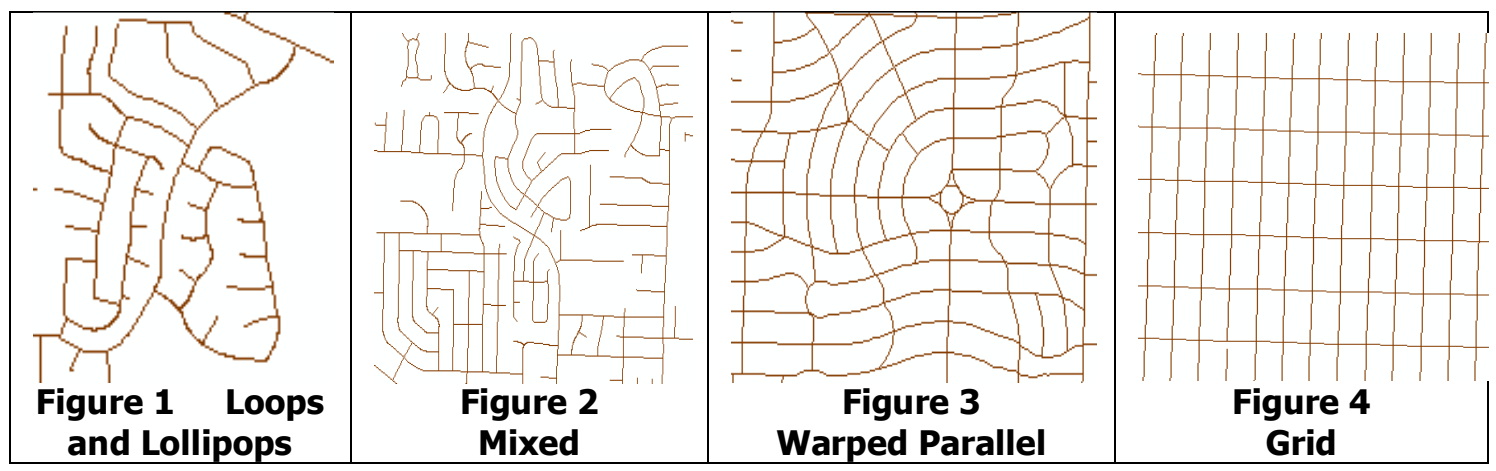

The papers reviewed are summarized in Table 1 . These have explored many urban form factors and their relationship to crashes in many different cities. Exposure, connectivity, accessibility, demographic factors, land use, and origin and destination factors were of interest. Multicollinearity amongst factors has led to difficulties with the modeling, and is thus an issue to be aware of in this type of study.

Choice of spatial unit has also created difficulties. TAZ and block groups were often used as spatial units. These spatial analysis units were not of equal size, requiring them to be normalized for comparison. TAZ and block groups are typically bounded by roadways, which makes treatment of crashes on the peripheral roadways an issue: one study counted them in each adjacent zone, effectively double counting those crashes; another study removed those crashes 
from the study. A consistent way to handle all crashes is needed. Kim utilized a uniform spatial grid and showed ArcGIS to be a useful crash analysis tool, but crash location was still typically tied to the nearest intersection which may skew analysis of urban form. It would be illuminating to study crashes at their specific geo-coded location, all included, all equally weighted.

This study will look at crash data for Portland, Oregon, considering traffic safety related to exposure, connectivity, transit accessibility, demographic, and origin and destination factors. It will utilize a uniform spatial grid as did Kim et al (13) for spatial unit, to allow inclusion of all crashes equally weighted without double counting. This should provide insight into overall local traffic safety, rather than only within developed neighborhoods. 
Table 1 Summary of Studies Reviewed

\begin{tabular}{|c|c|c|c|c|c|c|}
\hline $\begin{array}{l}\text { Resear- } \\
\text { chers }\end{array}$ & \begin{tabular}{|l|} 
Spatial \\
Unit \\
\end{tabular} & $\begin{array}{l}\text { Crash } \\
\text { data }\end{array}$ & $\begin{array}{l}\text { Other factors, } \\
\text { parameters }\end{array}$ & $\begin{array}{l}\text { Analysis } \\
\text { Method }\end{array}$ & Findings & Conclusions \\
\hline $\begin{array}{l}\text { Marks } \\
1957 \\
\text { LA County }\end{array}$ & $\begin{array}{l}86 \text { sub- } \\
\text { division } \\
\text { tracts }\end{array}$ & $\begin{array}{l}5 \text { years } \\
\text { crash data, } \\
\text { - only right } \\
\text { angle } \\
\text { crashes, } \\
\text { - major } \\
\text { bordering } \\
\text { streets not } \\
\text { included }\end{array}$ & $\begin{array}{l}\text { Did not control } \\
\text { for } \\
\text { traffic volume, } \\
\text { nor consider } \\
\text { land use } \\
\text { arrangements }\end{array}$ & $\begin{array}{l}\text { Compari- } \\
\text { son of } \\
\text { crash } \\
\text { rates }\end{array}$ & $\begin{array}{l}\text { Most crashes were at } \\
\text { intersections: } \\
4 \text { leg intersections higher } \\
\text { crash frequency than } \\
3 \text { leg intersections. } \\
\text { Irregularities increased crash } \\
\text { rates. }\end{array}$ & $\begin{array}{l}\text { Elements for safe design: } \\
\text { limited access design } \\
\text { avoid continuous through } \\
\text { streets } \\
\text { collectors exit onto only } \\
\text { one major street } \\
\text { prefer T over } 4 \text { leg } \\
\text { intersections } \\
\text { avoid irregularities }\end{array}$ \\
\hline $\begin{array}{l}\text { Kim, } \\
\text { Levine, Nitz } \\
1995 \\
\text { Oahu } \\
\end{array}$ & \begin{tabular}{|l|}
363 \\
census \\
block \\
groups \\
\end{tabular} & $\begin{array}{l}1990 \text { Oahu } \\
\text { crashes }\end{array}$ & & $\begin{array}{l}\text { Spatial } \\
\text { mapping }\end{array}$ & $\begin{array}{l}\text { Spatial pattern of crashes } \\
\text { varied by time of day, day of } \\
\text { week, vehicle type. }\end{array}$ & $\begin{array}{l}\text { Spatial mapping of crashes } \\
\text { could be useful for } \\
\text { community safety } \\
\text { improvement planning. }\end{array}$ \\
\hline $\begin{array}{l}\text { Kim and } \\
\text { Levine } \\
1996 \\
\text { Oahu }\end{array}$ & $\begin{array}{l}\text { Looked } \\
\text { at point, } \\
\text { segment, } \\
\text { and zonal } \\
\text { analysis }\end{array}$ & $\begin{array}{l}\text { Crash data } \\
\text { geocoded }\end{array}$ & & $\begin{array}{l}\text { Compared } \\
\text { actual to } \\
\text { predicted } \\
\text { crash rate, } \\
\text { spatially } \\
\text { mapped } \\
\end{array}$ & $\begin{array}{l}\text { 43\% crashes occurred at } \\
\text { intersections. }\end{array}$ & $\begin{array}{l}\text { GIS data useful in looking at } \\
\text { spatial relationships of crash } \\
\text { data }\end{array}$ \\
\hline $\begin{array}{l}\text { Ossen- } \\
\text { bruggen } \\
\text { Pendharkar } \\
\text { Ivan } \\
\quad 2001 \\
\quad \text { Stafford } \\
\text { Count, } \mathrm{NH} \\
\end{array}$ & $\begin{array}{l}87 \text { sites } \\
\text { on rural } \\
\text { and } \\
\text { suburban } \\
\text { two lane } \\
\text { undivided } \\
\text { highways } \\
\end{array}$ & $\begin{array}{l}1993-1997, \\
892 \\
\text { crashes }\end{array}$ & $\begin{array}{l}\text { Road } \\
\text { measurements } \\
\text { and land use, } \\
\text { driver behavior }\end{array}$ & $\begin{array}{l}\text { Ranked } \\
\text { site } \\
\text { hazard by } \\
\text { crash rate } \\
\text { Logistic } \\
\text { regression }\end{array}$ & $\begin{array}{l}\text { Village (mixed use) pedestrian } \\
\text { friendly sites less hazardous } \\
\text { than residential and shopping } \\
\text { sites without pedestrian } \\
\text { amenities such as sidewalks. }\end{array}$ & $\begin{array}{l}\text { Land use activity, pedestrian } \\
\text { friendliness, and } \\
\text { infrastructure more effective } \\
\text { at reducing road hazard than } \\
\text { traffic controls or posted } \\
\text { speed limitations. }\end{array}$ \\
\hline $\begin{array}{l}\text { Hadayeghi } \\
2003 \\
\text { Toronto, } \\
\text { Ontario, CA }\end{array}$ & $\begin{array}{l}463 \\
\text { traffic } \\
\text { zones }\end{array}$ & $\begin{array}{l}1996 \text { crash } \\
\text { data, } \\
\text { geocoded }\end{array}$ & $\begin{array}{l}\text { Socio- } \\
\text { economic, } \\
\text { demographic, } \\
\text { traffic demand, } \\
\text { network data }\end{array}$ & $\begin{array}{l}\text { Negative } \\
\text { binomial } \\
\text { regression }\end{array}$ & $\begin{array}{l}\text { Crash rate related to roadway } \\
\text { length, number of } \\
\text { intersections, employment, } \\
\text { household population, posted } \\
\text { speed limit, and higher } \\
\text { congestion levels. }\end{array}$ & $\begin{array}{l}\text { Predictive models developed } \\
\text { relating crash rate to various } \\
\text { parameters. } \\
\text { - Geometry of a } \\
\text { neighborhood design may } \\
\text { also be significant. }\end{array}$ \\
\hline
\end{tabular}


Table 1 Summary of Studies Reviewed, continued

\begin{tabular}{|c|c|c|c|c|c|c|}
\hline $\begin{array}{l}\begin{array}{l}\text { Resear- } \\
\text { chers }\end{array} \\
\end{array}$ & $\begin{array}{l}\text { Spatial } \\
\text { Unit } \\
\end{array}$ & Crash data & $\begin{array}{l}\text { Other factors, } \\
\text { parameters }\end{array}$ & $\begin{array}{l}\text { Analysis } \\
\text { Method }\end{array}$ & Findings & Conclusions \\
\hline $\begin{array}{l}\text { Ladron de } \\
\text { Guevara, } \\
\text { Washington } \\
\text { Oh } \\
\quad 2004 \\
\text { Tucson, AZ }\end{array}$ & 859 TAZ & $\begin{array}{l}1998-1999 \\
\text { crash } \\
\text { frequency } \\
\text { and severity }\end{array}$ & $\begin{array}{l}\text { Population for } \\
\text { exposure risk; } \\
\text { demographic, } \\
\text { including } \\
\text { schools, } \\
\text { job density; } \\
\text { bus stops; } \\
\text { bike routes, } \\
\text { road miles }\end{array}$ & $\begin{array}{l}\text { Negative } \\
\text { binomial } \\
\text { regression }\end{array}$ & $\begin{array}{l}\text { Fatal crash model found } \\
\text { population significant. } \\
\text { Injury crash significant } \\
\text { parameters: population } \\
\text { density, intersection density, } \\
\text { employment, miles arterials } \\
\text { and collector roadways. }\end{array}$ & $\begin{array}{l}\text { Planning level models can be } \\
\text { developed and useful for } \\
\text { MPO safety forecasting. } \\
\text { Population appears to better } \\
\text { represent exposure to risk } \\
\text { than VMT for this type } \\
\text { model. }\end{array}$ \\
\hline $\begin{array}{l}\text { Al-Masaeid } \\
\text { and } \\
\text { Suleiman } \\
\quad 2004 \\
\text { Damascus, } \\
\quad \text { Syria }\end{array}$ & $\begin{array}{l}14 \text { urban } \\
\text { zones }\end{array}$ & $2001-2002$ & $\begin{array}{l}\text { VMT, } \\
\text { population, land } \\
\text { use, street } \\
\text { network }\end{array}$ & $\begin{array}{l}\text { Multivariate } \\
\text { regression } \\
\text { analysis }\end{array}$ & $\begin{array}{l}\text { Grid networks have more } \\
\text { intersections and higher } \\
\text { crash rates than limited } \\
\text { access elements: urban } \\
\text { crashes are exponentially } \\
\text { proportional to intersection } \\
\text { density, total street length. }\end{array}$ & $\begin{array}{l}\text { Reducing the need for travel } \\
\text { reduces exposure and traffic, } \\
\text { thus reduces crash risk. } \\
\text { Commercial frontage away } \\
\text { from major thoroughfares } \\
\text { may reduce crashes. }\end{array}$ \\
\hline $\begin{array}{l}\text { Kim, } \\
\text { Brunner, } \\
\text { Yamashita } \\
\\
2006 \\
\text { Oahu } \\
\end{array}$ & $\begin{array}{l}\text { Uniform } \\
\text { grid }\end{array}$ & $\begin{array}{l}\text { Vehicle, } \\
\text { bike, and } \\
\text { pedestrian } \\
\text { crashes }\end{array}$ & $\begin{array}{l}\text { Land use, } \\
\text { employment, } \\
\text { economic, } \\
\text { population, } \\
\text { demographic }\end{array}$ & $\begin{array}{l}\text { Negative } \\
\text { binomial } \\
\text { regression }\end{array}$ & $\begin{array}{l}\text { Fatal and injury crash } \\
\text { parameters differed slightly, } \\
\text { related to population, age, } \\
\text { intersection density, } \\
\text { employment, and miles of } \\
\text { arterial and collector roads. }\end{array}$ & $\begin{array}{l}\text { Grid cell characteristics can } \\
\text { be used to statistically model } \\
\text { crash rates }\end{array}$ \\
\hline $\begin{array}{c}\text { Kim, Pant, } \\
\text { Yamashita } \\
\\
2010 \\
\text { Oahu }\end{array}$ & $\begin{array}{l}\text { Uniform } \\
\text { grid }\end{array}$ & $\begin{array}{l}2002-2004 \\
\text { crashes } \\
\text { assigned to } \\
\text { nearest } \\
\text { intersection, } \\
\text { freeway } \\
\text { crashes } \\
\text { excluded }\end{array}$ & $\begin{array}{l}\text { Demographic, } \\
\text { land use, } \\
\text { accessibility } \\
\text { measures }\end{array}$ & $\begin{array}{l}\text { Logistic } \\
\text { regression }\end{array}$ & $\begin{array}{l}\text { Statistical significance for } \\
\text { vulnerable populations, } \\
\text { disability, job count, land } \\
\text { use. Pedestrian crashes } \\
\text { increased with more bus } \\
\text { stops, bus route length. } \\
\text { Population only associated } \\
\text { with bicycle crashes. }\end{array}$ & $\begin{array}{l}\text { Crash predictions useful in } \\
\text { developing locations needing } \\
\text { safety improvement } \\
\text { strategies through } \\
\text { enforcement, engineering, or } \\
\text { education. } \\
\text { Multicollinear relations with } \\
\text { variables such as population } \\
\text { size, vulnerable population. }\end{array}$ \\
\hline
\end{tabular}


Table 1 Summary of Studies Reviewed, continued

\begin{tabular}{|c|c|c|c|c|c|c|}
\hline Researchers & $\begin{array}{l}\text { Spatial } \\
\text { Unit }\end{array}$ & Crash data & $\begin{array}{l}\text { Other factors, } \\
\text { parameters }\end{array}$ & $\begin{array}{l}\text { Analysis } \\
\text { Method }\end{array}$ & Findings & Conclusions \\
\hline $\begin{array}{l}\text { Clifton, } \\
\text { Burnier, and } \\
\text { Akar } \\
\quad 2009 \\
\text { Baltimore, MD }\end{array}$ & $\begin{array}{l}\text { disaggregat } \\
\text { e data, } 1 / 4 \\
\text { mile buffer } \\
\text { around } \\
\text { each crash } \\
\text { location }\end{array}$ & $\begin{array}{l}\text { Pedestrian } \\
\text { vehicle } \\
\text { crashes } \\
2000-2004 \\
\text { geocoded to } \\
\text { nearest } \\
\text { intersection }\end{array}$ & $\begin{array}{l}\text { Street network, } \\
\text { transit access, } \\
\text { land use, vehicle } \\
\text { type, weather, } \\
\text { road condition, } \\
\text { sex, population, } \\
\text { substance abuse }\end{array}$ & $\begin{array}{l}\text { Probit } \\
\text { model }\end{array}$ & $\begin{array}{l}\text { Transit access and pedestrian } \\
\text { connectivity were the only } \\
\text { built environment variables } \\
\text { significant in the analysis. } \\
\text { Used Herfindahl-Hirschmann } \\
\text { index measure of land use } \\
\text { mix. }\end{array}$ & $\begin{array}{l}\text { Areas with low } \\
\text { connectivity and transit } \\
\text { access need greater } \\
\text { safety interventions, } \\
\text { injuries there are more } \\
\text { severe. }\end{array}$ \\
\hline $\begin{array}{l}\text { Dumbaugh } \\
\text { and Rae } \\
\\
2009 \\
\text { San Antonio, } \\
\text { TX }\end{array}$ & $\begin{array}{l}747 \text { census } \\
\text { block } \\
\text { groups plus } \\
\text { buffer to } \\
\text { include } \\
\text { periphery } \\
\text { streets }\end{array}$ & $\begin{array}{l}2004-2006 \\
\text { crashes, } \\
\text { on and off } \\
\text { roadway; } \\
\text { peripheral } \\
\text { crashes } \\
\text { included and } \\
\text { possibly } \\
\text { double } \\
\text { counted }\end{array}$ & $\begin{array}{l}\text { Parcel-level land } \\
\text { use data, } \\
\text { demographic } \\
\text { data; roadway } \\
\text { network data } \\
\text { (street miles, } \\
\text { number of } 3,4 \\
\text { leg intersections) }\end{array}$ & $\begin{array}{l}\text { Negative } \\
\text { binomial } \\
\text { regression }\end{array}$ & $\begin{array}{l}\text { Traffic re-located away from } \\
\text { residential areas reduces } \\
\text { neighborhood traffic volume. } \\
\text { Arterials designed with wide } \\
\text { lanes and long sight distances } \\
\text { for higher speeds should have } \\
\text { limited access, with } \\
\text { commercial traffic on lower } \\
\text { speed thoroughfares. }\end{array}$ & $\begin{array}{l}\text { There is an important } \\
\text { relationship between } \\
\text { community design and } \\
\text { traffic safety. } \\
\text { Designing pedestrian } \\
\text { scale, livable streets } \\
\text { emphasizes access over } \\
\text { mobility, and has better } \\
\text { traffic safety. }\end{array}$ \\
\hline $\begin{array}{l}\text { Rifaat and Tay } \\
2009 \\
\text { Calgary, } \\
\text { Alberta } \\
\text { Canada }\end{array}$ & $\begin{array}{l}227 \\
\text { community } \\
\text { areas }\end{array}$ & $\begin{array}{l}2003-2005 \\
\text { two vehicle } \\
\text { crashes; } \\
\text { - no crashes } \\
\text { on boundary } \\
\text { roadways }\end{array}$ & $\begin{array}{l}4 \text { street patterns: } \\
\text { - gridiron, } \\
\text { - warped parallel, } \\
\text { - loop \& lollipop, } \\
\text { - mixed }\end{array}$ & $\begin{array}{l}\text { Logistic } \\
\text { regression }\end{array}$ & $\begin{array}{l}\text { Roadway and demographic } \\
\text { data provided control } \\
\text { relationships to crash rates. } \\
\text { - Crashes on boundary } \\
\text { roadways not considered due } \\
\text { to boundary problem }\end{array}$ & $\begin{array}{l}\text { Compared to gridiron, } \\
\text { loops and lollipops } \\
\text { design has decreased } \\
\text { crash injury risk. }\end{array}$ \\
\hline $\begin{array}{l}\text { Rifaat and Tay } \\
2010 \\
\text { Calgary, } \\
\text { Alberta } \\
\text { Canada }\end{array}$ & $\begin{array}{l}227 \\
\text { community } \\
\text { areas }\end{array}$ & $\begin{array}{l}2003-2005 \\
\text { two vehicle } \\
\text { crashes; } \\
\text { crashes on } \\
\text { boundary } \\
\text { roadways } \\
\text { not } \\
\text { considered }\end{array}$ & $\begin{array}{l}\text { *4 street patterns } \\
\text { - gridiron, } \\
\text { - warped parallel, } \\
\text { - loop \& lollipop, } \\
\text { - mixed } \\
\text { * Road condition, } \\
\text { demographic, } \\
\text { socioeconomic, } \\
\text { land use; }\end{array}$ & $\begin{array}{l}\text { Negative } \\
\text { binomial } \\
\text { regression }\end{array}$ & $\begin{array}{l}\text { Crash data converted into } \\
\text { EPDO (equivalent property } \\
\text { damage only) crashes. } \\
\text { * AADT estimated from ITE } \\
\text { trip generation models. } \\
\text { * Control factors affected } \\
\text { crash rates; socioeconomic } \\
\text { and demographic factors also } \\
\text { statistically significant }\end{array}$ & $\begin{array}{l}\text { Limited access street } \\
\text { patterns had lower } \\
\text { crash rates than } \\
\text { gridiron layouts. }\end{array}$ \\
\hline
\end{tabular}




\subsection{DATA SOURCES AND PREPARATION}

Prior work had shown that important data for studying the relationship between urban form and traffic safety were of course crash data, as well as choice of spatial unit, and how crashes along roadways at the periphery of spatial units were handled. Other important factors fell into categories of exposure (traffic volume), connectivity (street length, number and types of intersections), accessibility to transit, demographics (population), and origin and destination (employment and businesses). Data sources and preparation of these sources are discussed in the following sections.

\subsection{Data Sources}

\subsubsection{Crash Data}

This study looked at reported crashes from 2005-2007 within the Portland, Oregon city limit (as defined in 2007). Crash data were available from the City of Portland, geo-coded by crash location. Further crash details were found using OrTSDA, the Oregon Traffic Safety Data Archive, which is a mirror of the statewide Crash Data System (CDS) maintained by ODOT (Oregon Department of Transportation). Crash data from a three-year period were used in order to have a large data set while limiting the likelihood of structural changes over the study period. 
Crash reporting varies from state to state. Oregon is a self-reporting state for crashes, where the individual drivers are required to file an Oregon Traffic Accident and Insurance Report within 72 hours if they are involved in a crash that results in injury, death, more than $\$ 1,500$ damage to their vehicle, or more than $\$ 1,500$ damage and towing of another vehicle. While police officers do complete and file reports, many non-injury, property-damage-only (PDO) crash reports do not include a police officer's written report. These reporting disparities mean that many PDO crashes are not reported in Oregon.

Freeway crashes were eliminated from the data. A freeway is a very different type of transportation infrastructure, which would be expected to have very different effects on traffic safety than the local streets which were the focus of this study.

\subsubsection{Exposure}

Exposure data tend to be crucial in crash analysis: the more exposure to use of the transportation system, the greater the probability of crashes. Metro, the regional government in the Portland metropolitan area, provided ArcGIS layers from the regional travel demand model that included 2005 exposure data on volume to capacity ratios $(\mathrm{v} / \mathrm{c})$, peak hour volume, VMT (vehicle miles traveled), and average free speed. Although available for only some streets, these data were felt to give relative data for analysis and comparison. 


\subsubsection{Connectivity}

Connectivity data were a chance to represent the street network layout. The street network scale developed by Rifaat and Tay was applied to all 1284 spatial grid cells; some had too little street network to have one of the four network types assigned. Other data expected to shed light on connectivity included road network length for street or arterials, as well as counts of intersections and how many legs to the intersections,

Metro maintains a rich geo-spatial database, the Regional Land Use and Information System (RLIS). The RLIS 2009 dataset at PSU (Portland State University) was used for data on streets and roadway. Road network data included layers with lines showing the location of streets, minor arterials, major arterials, and freeways.

\subsubsection{Transit Accessibility}

Transit accessibility was expected to also inform this study. High transit usage could mean less vehicles on the road, and thus less exposure. Transit riders are often pedestrians either before or after their transit portion of a trip. Transit ridership data were obtained from 2007 TriMet data in the PORTAL database archive at PSU. Transit stops, routes, and schools were available in the RLIS 2009 dataset. 


\subsubsection{Demographics, Origins, and Destinations}

Population has been shown to be a strong factor in previous crash data studies, and is often used in transportation modeling and forecasting. Employment is a strong factor in trip generation, since most workers need to commute to the workplace. The number of businesses was of interest since businesses attract not only customers and employees to make trips, but the number of business establishments also affects the number of driveways along roadways which increases likelihood of conflict and potential for crashes.

The RLIS database included demographic data on population, housing units, and dwelling units. Metro shape files provided 2005 employment data (number of employees) by TAZ (transportation analysis zones), and modeled block size in raster layers.

Business data were obtained from www.ReferenceUSA.com for grocery, clothing, goods, services (beauty, laundry, mail, bank), fitness, entertainment, food, schools \& academies, religious institutions. The businesses were then geocoded by address so that the number of businesses could be counted 


\subsection{Preparation}

\subsubsection{Definition of Spatial Analysis Unit}

ArcGIS was used to illuminate and aggregate the data. A uniform spatial grid of $\sim 0.1$ square miles (1670 feet long on a side) was set over the Portland metropolitan area to be used as the spatial unit for analysis, each spatial unit inherently of the same area. This allowed all crashes to be included without double counting, since there would be little likelihood that spatial grid boundaries would fall on roadway locations, unlike the use of TAZ or census block group spatial units, which typically are bounded by roadways. The grid was limited to whole cells within the 2007 Portland City Limits for a total of 1284 cells.

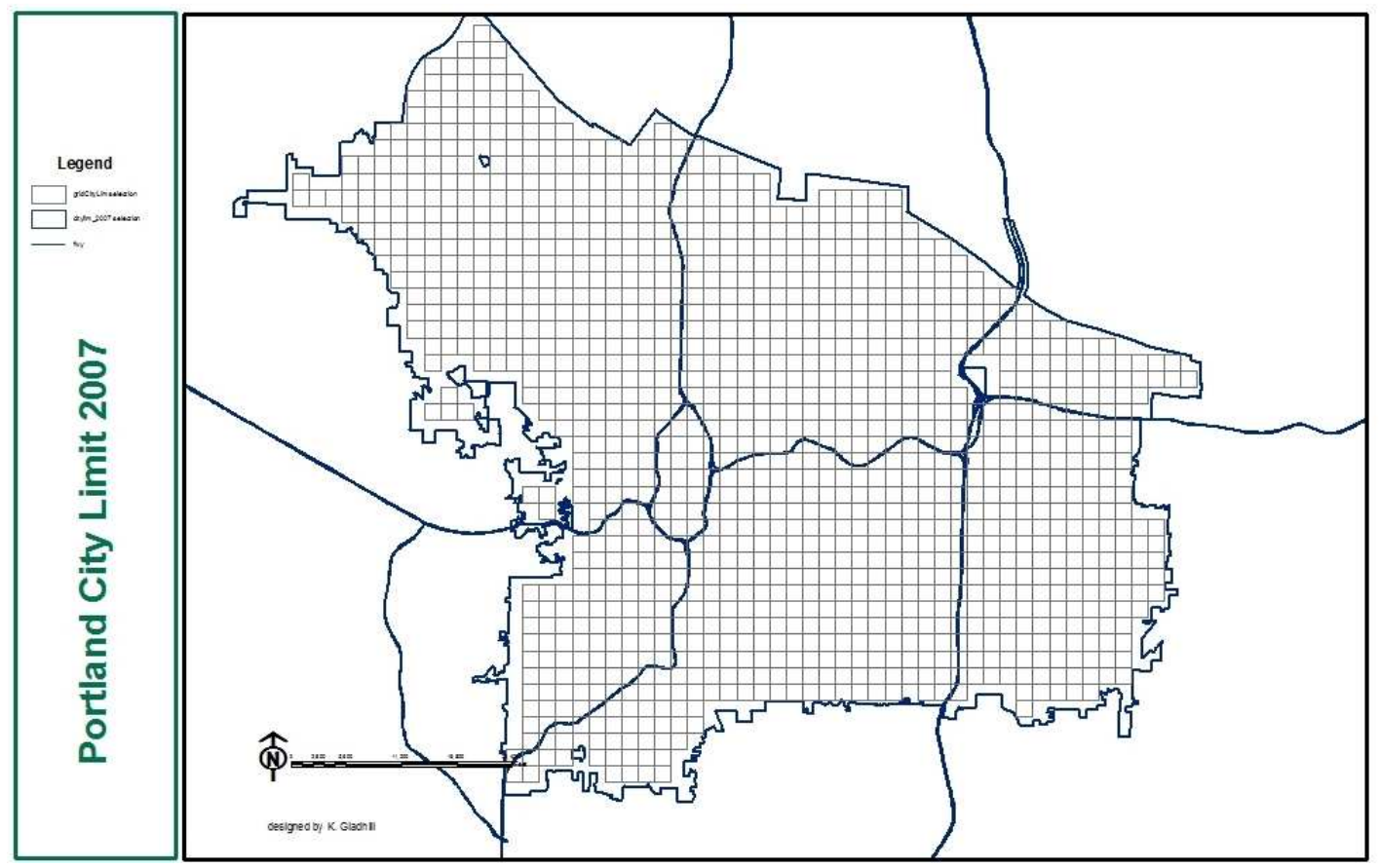

Figure 5 Spatial Data Grid within the Portland City Limits

Page 21 


\subsubsection{Data Aggregation}

Crash data were allocated into the spatial grid cells using ArcGIS. Exposure, connectivity, transit accessibility, demographic, and origin and destination data were also aggregated into the grid cells: the point data were summed by count, line data summed for total length, and polygon data summed proportional to the percentage of the polygon's area that was within a grid cell.

$$
\begin{aligned}
\text { Point data: } & \text { cell value }=\sum_{k=0}^{n} x \\
\text { Line data: } & \text { cell value }=\sum_{k=0}^{n}(\text { line length } \text { within } \text { grid }) \\
\text { Polygon data: } & \text { cell value }=\sum_{k=0}^{n} \quad \text { pctArea }
\end{aligned}
$$

where pctArea $=(\%$ polygon in grid cell $) *($ factor value for polygon $)$

As an example, a spatial grid cell containing $30 \%$ of the area of a TAZ spatial unit with a factor value of 120 would get $30 \%$ of the TAZ value for a factor, or 36. The proportion of that factor for all other TAZ units represented in the grid cell was similarly calculated, and the total summed to compute a value for that factor for that grid cell. Table 2 lists the data variables and how they were aggregated into the spatial grid cells.

Intersections were counted using ArcGIS to determine points where street lines intersected, and an algorithm then eliminated duplicates and tallied the number of intersection legs. 
Table 2 Data aggregated into Uniform Spatial grid Cells for Study

\begin{tabular}{|c|c|c|c|}
\hline DATA & \begin{tabular}{|l} 
Description \\
\end{tabular} & Aggregation & SOURCE \\
\hline Crash data & Portland, OR 2005-2007 & sum of point data & City of Portland, OrTSDA \\
\hline \multicolumn{4}{|c|}{ CONNECTIVITY } \\
\hline Street Network & $\begin{array}{l}4=\text { grid } \\
3=\text { warped parallel } \\
2=\text { mixed } \\
1=\text { loops and lollipops } \\
0=\text { could not determine }\end{array}$ & $\begin{array}{l}\text { values assigned to } \\
\text { each spatial grid cell }\end{array}$ & $\begin{array}{l}\text { evaluated by researcher } \\
\text { inspecting each spatial } \\
\text { grid cell }\end{array}$ \\
\hline Intersections & $\begin{array}{l}\text { Count of total } \\
\text { intersections }\end{array}$ & $\begin{array}{l}\text { intersections per } \\
\text { spatial grid cell }\end{array}$ & $\begin{array}{l}\text { RLIS 2009, } \\
\text { intersection analysis }\end{array}$ \\
\hline Streets & $\begin{array}{l}\text { Total street length, } \\
\text { arterial length, major } \\
\text { arterial length, freeway } \\
\text { length }\end{array}$ & $\begin{array}{l}\text { sum of street length } \\
\text { (line data) in spatial } \\
\text { grid cell }\end{array}$ & RLIS 2009 \\
\hline \multicolumn{4}{|c|}{ TRANSIT ACCESSIBILITY } \\
\hline Transit stops & Count of transit stops & sum of point data & RLIS 2009 \\
\hline Transit route & Total transit route length & $\begin{array}{l}\text { sum line data in } \\
\text { spatial grid cell }\end{array}$ & RLIS 2009 \\
\hline Ons and Offs & $\begin{array}{l}\text { Total transit count of } \\
\text { riders getting on and off }\end{array}$ & $\begin{array}{l}\text { sum of values in } \\
\text { spatial grid cell }\end{array}$ & PORTAL 2007 data \\
\hline \multicolumn{4}{|l|}{ EXPOSURE } \\
\hline $\mathrm{v} / \mathrm{c}$ & $\begin{array}{l}\text { Volume to capacity ratios } \\
\text { on some roadways }\end{array}$ & $\begin{array}{l}\text { average of values in } \\
\text { spatial grid cell }\end{array}$ & $\begin{array}{l}\text { Metro } 2005 \\
\text { Transportation Model }\end{array}$ \\
\hline VMT & Vehicle miles traveled & $\begin{array}{l}\text { sum of values in } \\
\text { spatial grid cell }\end{array}$ & $\begin{array}{l}\text { Metro } 2005 \\
\text { Transportation Model }\end{array}$ \\
\hline Avg free speed & 2006 data & $\begin{array}{l}\text { average of values in } \\
\text { spatial grid cell }\end{array}$ & $\begin{array}{l}\text { Metro } 2005 \\
\text { Transportation Model }\end{array}$ \\
\hline Schools & 2006 data & sum of point data & RLIS 2009 \\
\hline \multicolumn{4}{|c|}{ DEMOGRAPHICS, ORIGINS, and DESTINATIONS } \\
\hline Population & 2006 data & $\begin{array}{l}\text { apportioned ratio of } \\
\text { area in spatial grid } \\
\text { cell to factor value }\end{array}$ & RLIS 2009 \\
\hline Dwelling units & 2006 data & apportioned & RLIS 2009 \\
\hline Households & 2006 data & apportioned & RLIS 2009 \\
\hline Employment & 2006 data & apportioned & Metro \\
\hline Business & 2010 & sum of point data & ReferenceUSA.com \\
\hline Block Size & Raster file & $\begin{array}{l}\text { raster converted to } \\
\text { points, sum of } \\
\text { points in spatial grid } \\
\text { cell }\end{array}$ & Metro \\
\hline
\end{tabular}




\subsection{DESCRIPTIVE ANALYSIS}

This section will take an initial look at the data, with analysis of the crash data, and a qualitative look at exposure, connectivity, transit accessibility, demographic, and origin and destination data using chloropleths from ArcGIS.

\subsection{Crash Data}

The study dataset had a total of 21,492 non-freeway crashes within the city limits of Portland for the years 2005-2007. This number of crashes is considerably lower than more than 150,000 in the San Antonio study (3). This may be largely due to the underreporting issue with Oregon crash data. Looking at the crash data aggregated into the uniform spatial grid cells revealed that spatially crashes were concentrated in areas with heavy traffic: downtown, along high volume arterials, and adjacent to freeways (see Figure 6). Figure 7 shows a histogram of crash count per spatial grid cell for the study dataset; most cells had low crash counts. 


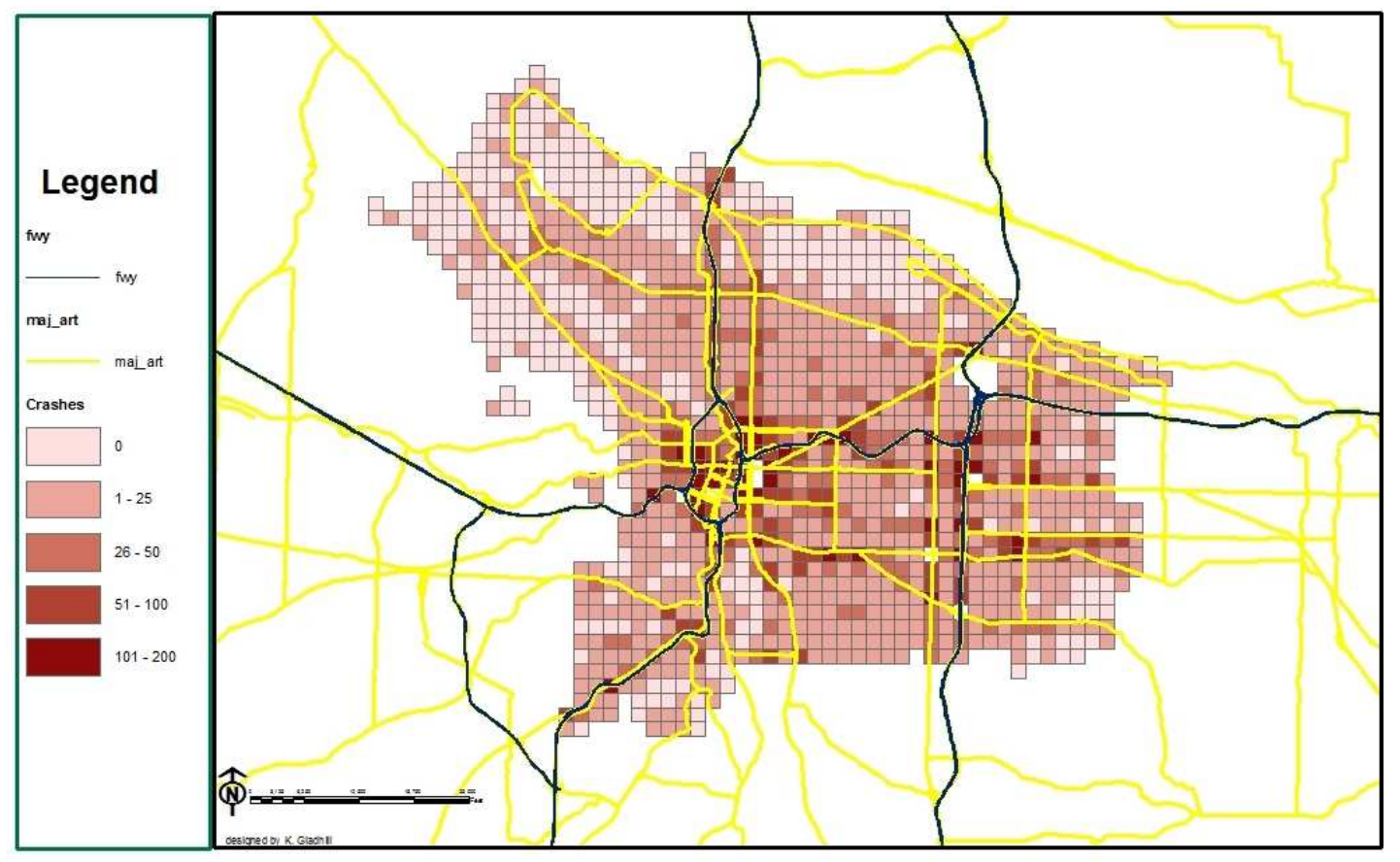

Figure 6 Total Non-freeway Crashes Portland 2005 - 2007

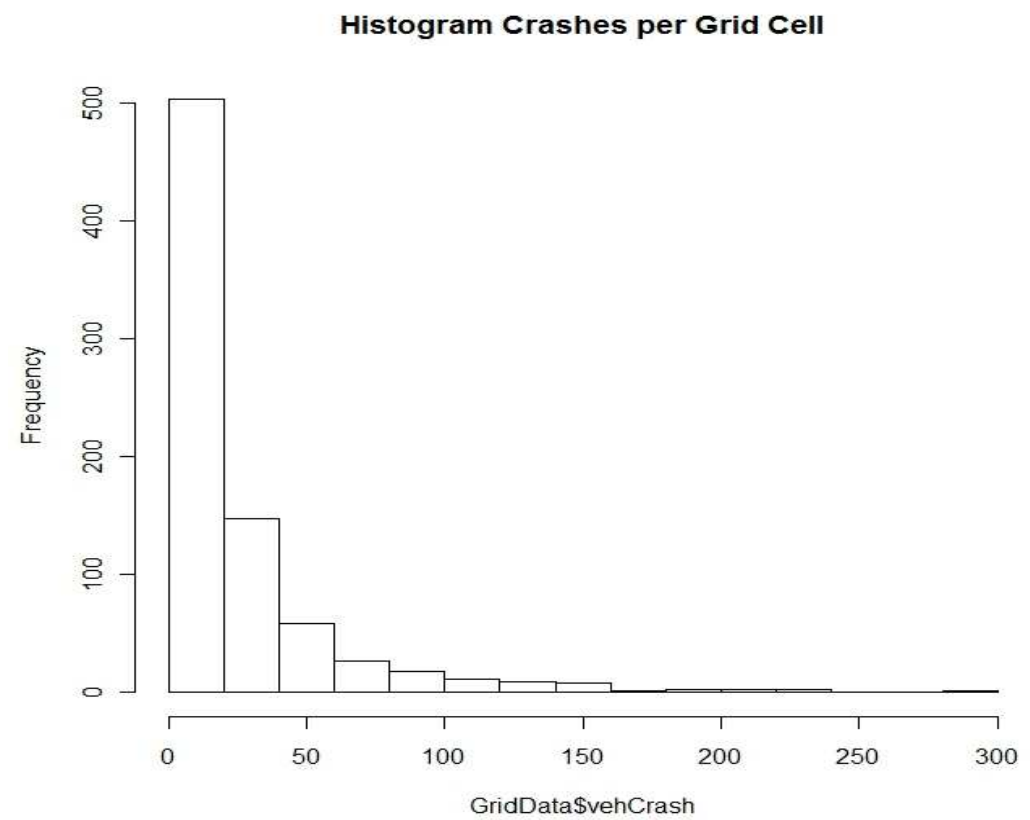

Figure 7 Histogram of 2006-2007 Crashes in Portland City Limits

Page 25 
Analysis of the crash data by crash types, see Figure 8, clearly showed that the four primary crash types were angle; stopped, where both vehicles were going the same direction; straight crashes where both vehicles were going the same direction; and turn crashes where one vehicle was turning in front of an oncoming vehicle going straight in the opposite direction.

Figure 9 illustrates each of these crash types. Angle crashes would have increased likelihood of occurrence the more cross streets to a roadway, causing a vehicle to cross in front of another vehicle, so would be expected to increase the more intersections. Stopped crashes, most likely rear ending, would be increased with traffic control bringing vehicles to a stop at intersections. Straight crashes would likely be caused by going too fast, thus overtaking another vehicle; or due to a sudden deceleration that the following vehicle did not respond to in time. Turn crashes would have increased likelihood with increased cross streets and driveways, where one vehicle would turn across an on-coming vehicle's path.

PDO (property damage only) crashes were most common (see Table 3) at 60.4 percent of the total crashes. Minor injury level C accounted for 21.6 percent of the total crashes, which was more than half of all injury crashes. Fixed object and pedestrian crash types accounted for 49 of the 82 fatalities. 


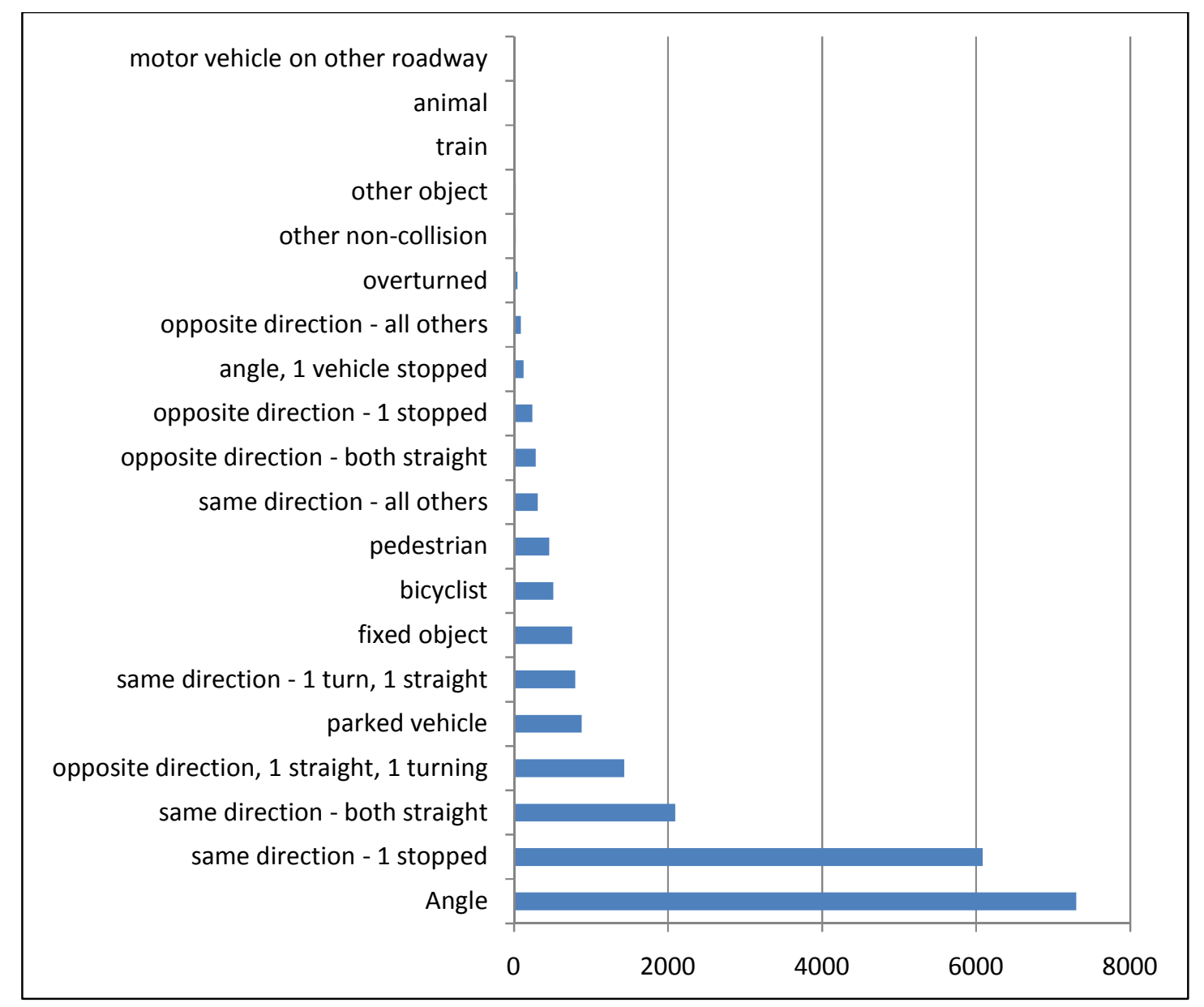

Figure $8 \quad 2005-2007$ Total Crashes by Crash Type
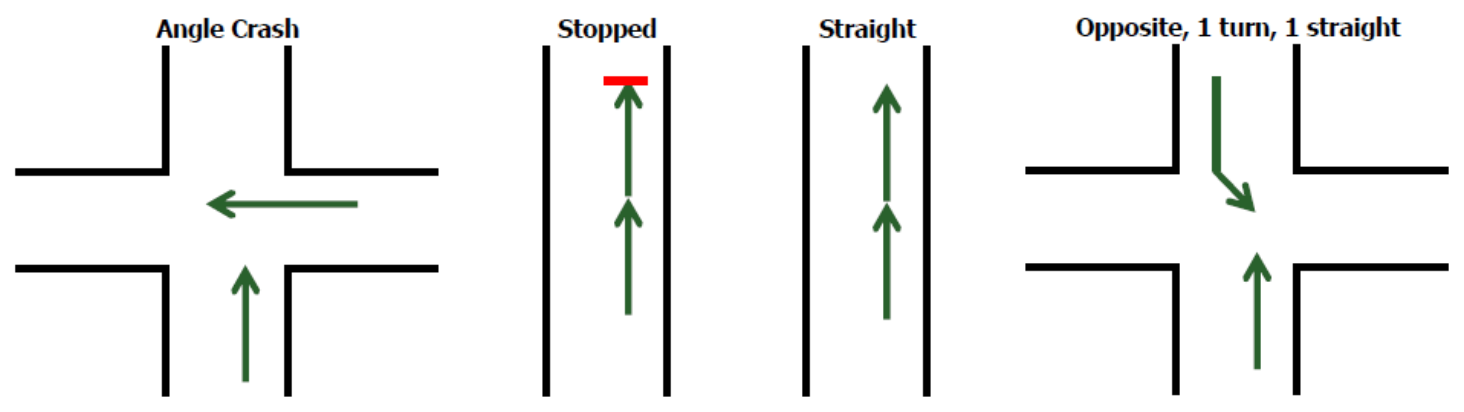

Figure 9 Primary Crash Types

Page 27 
Table 32005 - 2007 Portland Non-freeway Crashes in Spatial Grid Cells

\begin{tabular}{|c|c|c|c|c|c|c|c|}
\hline & Total & Fatality & Injury & INJ A & INJ B & INJ C & PDO \\
\hline Angle & 7303 & 9 & 2769 & 197 & 1200 & 1372 & 4525 \\
\hline $\begin{array}{r}\text { same direction - } \\
1 \text { stopped }\end{array}$ & 6086 & & 2675 & 75 & 743 & 1857 & 3411 \\
\hline $\begin{array}{r}\text { same direction - } \\
\text { both straight }\end{array}$ & 2094 & 4 & 507 & 17 & 148 & 342 & 1583 \\
\hline $\begin{array}{r}\text { opposite direction, } \\
1 \text { straight, } 1 \text { turning }\end{array}$ & 1431 & 1 & 617 & 48 & 242 & 327 & 813 \\
\hline parked vehicle & 881 & 3 & 177 & 22 & 81 & 74 & 701 \\
\hline $\begin{array}{l}\text { same direction - } \\
1 \text { turning, } 1 \text { straight }\end{array}$ & 797 & 2 & 174 & 5 & 46 & 123 & 621 \\
\hline fixed object & 757 & 24 & 293 & 54 & 136 & 103 & 440 \\
\hline bicyclist & 511 & 7 & 477 & 71 & 256 & 150 & 27 \\
\hline pedestrian & 457 & 25 & 427 & 83 & 204 & 140 & 5 \\
\hline $\begin{array}{r}\text { same direction - } \\
\text { all others }\end{array}$ & 310 & & 50 & 2 & 9 & 39 & 260 \\
\hline $\begin{array}{r}\text { opposite direction, } \\
\text { both straight }\end{array}$ & 285 & 6 & 127 & 23 & 58 & 46 & 152 \\
\hline $\begin{array}{r}\text { opposite direction, } \\
1 \text { stopped }\end{array}$ & 242 & & 30 & 2 & 9 & 19 & 212 \\
\hline $\begin{array}{r}\text { angle - } \\
1 \text { vehicle stopped }\end{array}$ & 128 & & 25 & 3 & 3 & 19 & 103 \\
\hline $\begin{array}{r}\text { opposite direction } \\
\text { all others }\end{array}$ & 92 & & 20 & & 6 & 14 & 72 \\
\hline overturned & 43 & 1 & 31 & 6 & 17 & 8 & 11 \\
\hline other non-collision & 25 & & 12 & 3 & 7 & 2 & 13 \\
\hline other object & 22 & & 5 & & 2 & 3 & 17 \\
\hline train & 20 & & 7 & 2 & & 5 & 13 \\
\hline animal & 4 & & 1 & & & 1 & 3 \\
\hline $\begin{array}{r}\text { motor vehicle on } \\
\text { other roadway }\end{array}$ & 4 & & & & & & 4 \\
\hline TOTAL & 21492 & 82 & 8424 & 613 & 3167 & 4644 & 12986 \\
\hline
\end{tabular}

INJ $A=$ injury level $A$, incapacitating

INJ $B=$ injury level $B$, non- incapacitating

INJ C = injury level C, possible injury

$\mathrm{PDO}=$ property damage only

More information on injury severity can be found at:

http://www.oregon.gov/ODOT/TD/TDATA/car/docs/2007CodeManualVersion2.0.pdf 


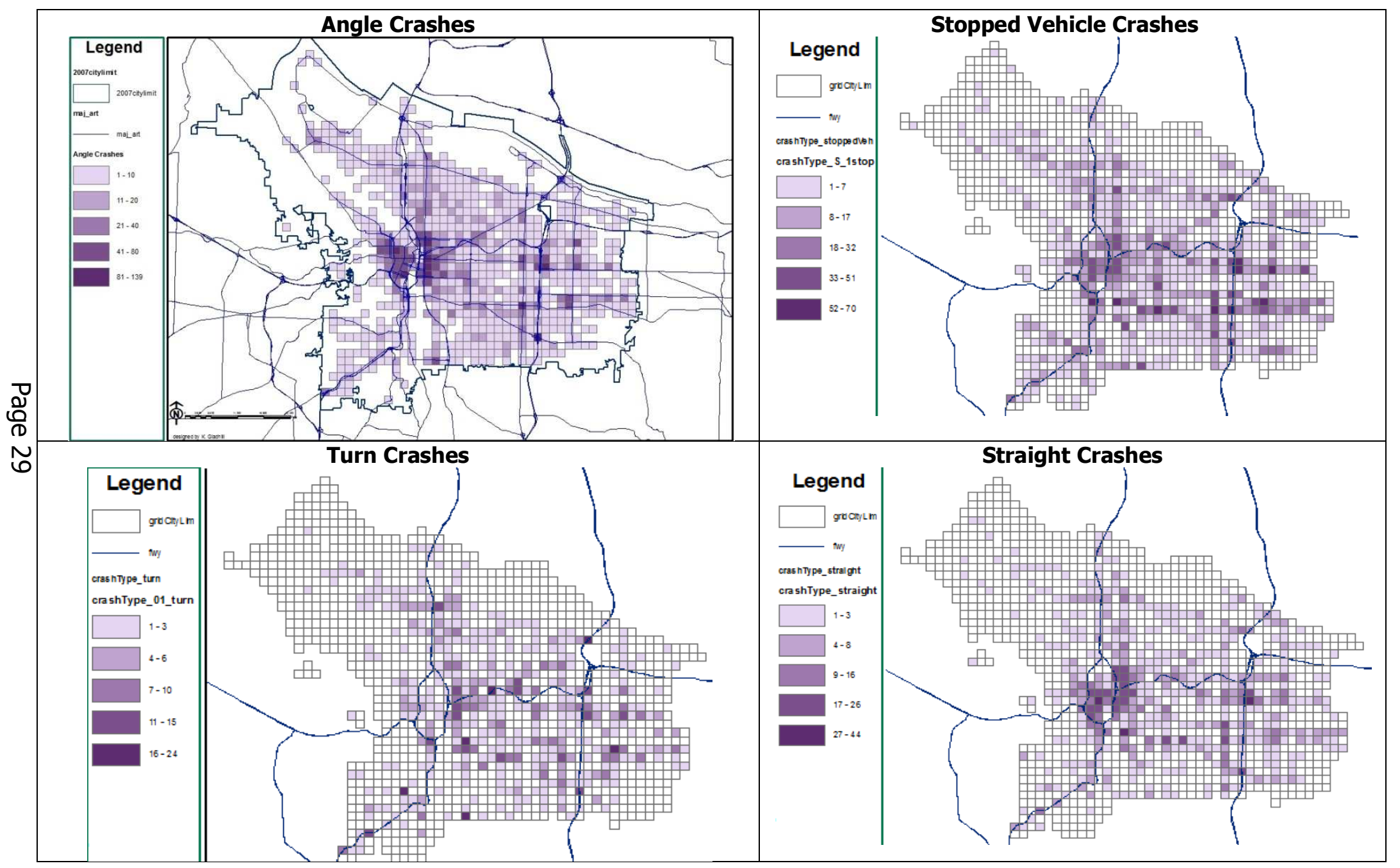

Figure 102005 - 2007 Vehicle Crashes by Crash Type, Spatial Maps 
Figure 10 shows that the spatial distribution of crashes differed for different crash types. Angle crashes, stopped vehicle crashes, and crashes where both vehicles were going straight showed similar distributions to total vehicle crashes with crashes concentrated along major arterial corridors. Turn crashes were more randomly distributed, although major arterial corridors are still discernible. High frequency in specific spatial grid cells indicates that specific locations may have problems which contribute to likelihood of each particular crash type.

The crash dataset included 503 pedestrian crashes and 523 bicycle crashes. These crashes are summarized by motor vehicle operator error, see Table 4 and Table 5. Failure to yield right of way (ROW) was the leading cause cited in the crash data for both pedestrian and bicycle crashes; presumably the vehicle failing to yield. Whereas greater than 60 percent of all vehicle crashes were PDO, pedestrian and bicycle crashes involved injury more than 93 percent of the time. Pedestrian crash fatality outcomes were more than ten times that of overall crash fatalities, and bicycle crash fatality rates were three times that of overall crashes.

Spatially, pedestrian crashes were concentrated downtown and along major arterial routes in the city, see Figure 11; bicycle crashes were more randomly distributed, see Figure 12 . 
Table 42005 - 2007 Pedestrian Crashes

\begin{tabular}{|c|c|c|c|c|c|c|c|}
\hline & Total & Fatality & Injury & INJ A & INJ B & INJ C & PDO \\
\hline $\begin{array}{l}\text { Failed to Yield } \\
\text { Right of Way }\end{array}$ & 294 & 13 & 277 & 42 & 140 & 95 & 4 \\
\hline $\begin{array}{l}\text { Non-motorist } \\
\text { illegally in roadway }\end{array}$ & 111 & 7 & 103 & 28 & 42 & 33 & 1 \\
\hline $\begin{array}{r}\text { Too fast for } \\
\text { conditions }\end{array}$ & 16 & 1 & 15 & 3 & 11 & 1 & \\
\hline Other driving error & 13 & & 13 & 3 & 7 & 3 & \\
\hline $\begin{array}{r}\text { Inattention } \\
\text { Disregarded }\end{array}$ & 12 & 2 & 10 & 2 & 3 & 5 & \\
\hline $\begin{array}{r}\text { Red-Amber-Green } \\
\text { traffic signal }\end{array}$ & 11 & & 11 & 2 & 8 & 1 & \\
\hline Careless & 10 & & 10 & 5 & 5 & & \\
\hline $\begin{array}{r}\text { Speed too fast for } \\
\text { conditions }\end{array}$ & 8 & 1 & 7 & 6 & & 1 & \\
\hline Not visible & 7 & 1 & 6 & 1 & 4 & 1 & \\
\hline Other & 6 & 2 & 4 & & 2 & 2 & \\
\hline Improper passing & 4 & 2 & 2 & & 2 & & \\
\hline Improper turn & 2 & & 2 & 2 & & & \\
\hline Reckless & 2 & & 2 & & & 2 & \\
\hline $\begin{array}{r}\text { Followed too } \\
\text { closely }\end{array}$ & 4 & & 4 & 2 & 2 & & \\
\hline Passed stop sign & 1 & & 1 & 1 & & & \\
\hline Fatigue & 1 & & 1 & & 1 & & \\
\hline no code applicable & 1 & & 1 & 1 & & & \\
\hline TOTAL & 503 & 29 & 469 & 98 & 227 & 144 & 5 \\
\hline & & $5.8 \%$ & $93.2 \%$ & $19.5 \%$ & $45.1 \%$ & $28.6 \%$ & $1.0 \%$ \\
\hline
\end{tabular}

Page 31 
Table $5 \quad 2005$ - 2007 Bicycle Crashes

\begin{tabular}{|c|c|c|c|c|c|c|c|}
\hline & Total & Fatality & Injury & INJ A & INJ B & INJ C & PDO \\
\hline Failed to Yield & & & & & & & \\
\hline Right of Way & 343 & 4 & 318 & 38 & 178 & 102 & 21 \\
\hline Disregarded Red- & & & & & & & \\
\hline Amber-Green & 47 & 1 & 43 & 10 & 17 & 16 & 3 \\
\hline $\begin{array}{l}\text { Non-motorist } \\
\text { illegally in roadway }\end{array}$ & 28 & & 27 & 5 & 13 & 9 & 1 \\
\hline Other & 20 & & 20 & 4 & 10 & 6 & \\
\hline Other driving error & 18 & 2 & 15 & & 9 & 6 & 1 \\
\hline Passed stop sign & 18 & & 18 & 4 & 10 & 4 & \\
\hline Improper turn & 13 & & 13 & 3 & 9 & 1 & \\
\hline Followed too & & & & & & & \\
\hline closely & 11 & & 10 & 5 & 3 & 2 & 1 \\
\hline Improper lane & & & & & & & \\
\hline change & 6 & & 6 & 1 & 4 & 1 & \\
\hline Inattention & 6 & & 6 & 3 & 1 & 2 & \\
\hline Careless & 4 & & 4 & 2 & 2 & & \\
\hline Improper passing & 3 & & 3 & 1 & 1 & 1 & \\
\hline Reckless & 3 & & 3 & & & 3 & \\
\hline Speed too fast for & & & & & & & \\
\hline conditions & 2 & 1 & 1 & & 1 & & \\
\hline Not visible & 1 & & 1 & & 1 & & \\
\hline \multirow[t]{2}{*}{ TOTAL } & 523 & 8 & 488 & 76 & 259 & 153 & 27 \\
\hline & & $1.5 \%$ & $93.3 \%$ & $14.5 \%$ & $49.5 \%$ & $29.3 \%$ & $5.2 \%$ \\
\hline
\end{tabular}

Page 32 


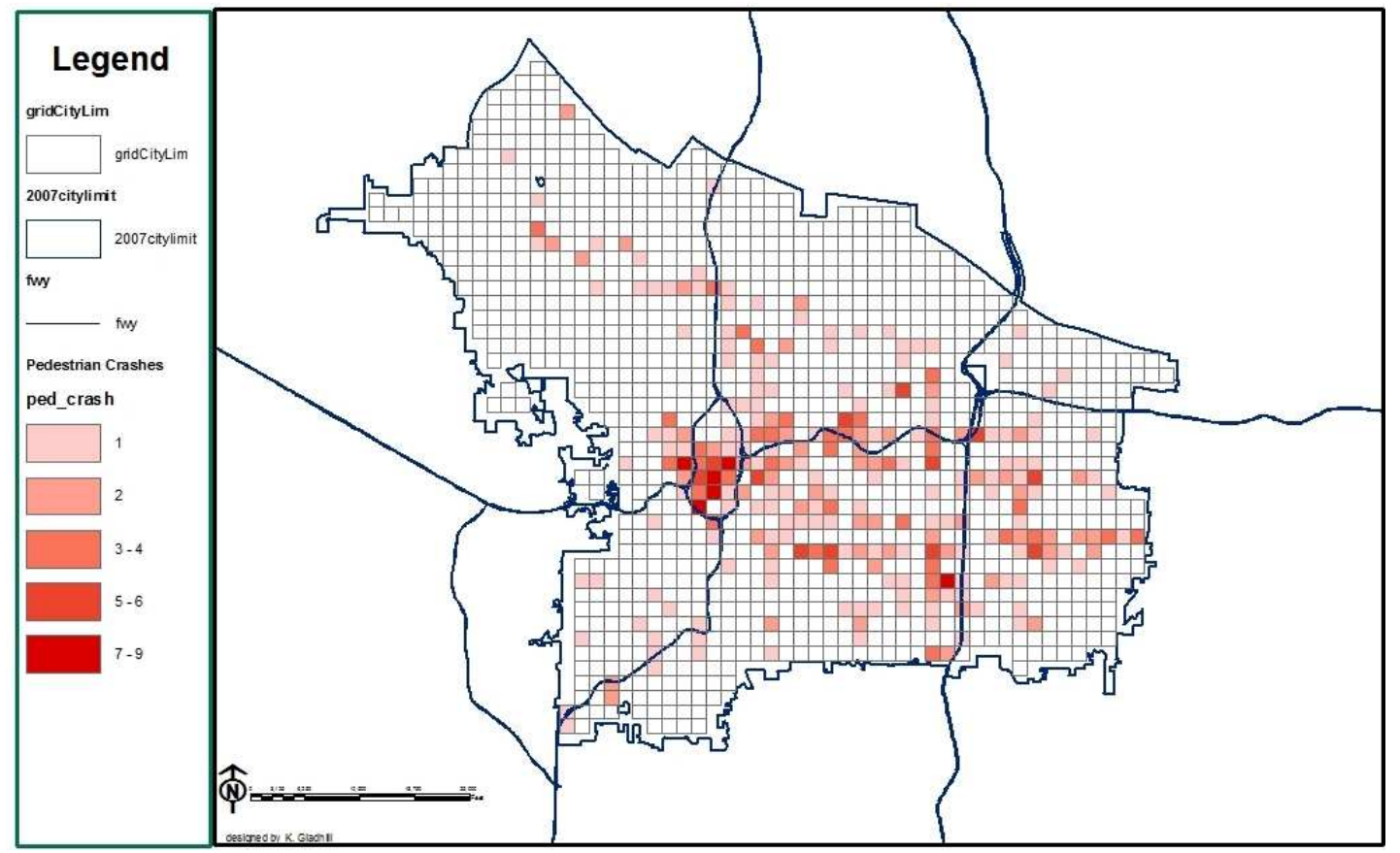

Figure 112005 - 2007 Pedestrian Crash Spatial Map

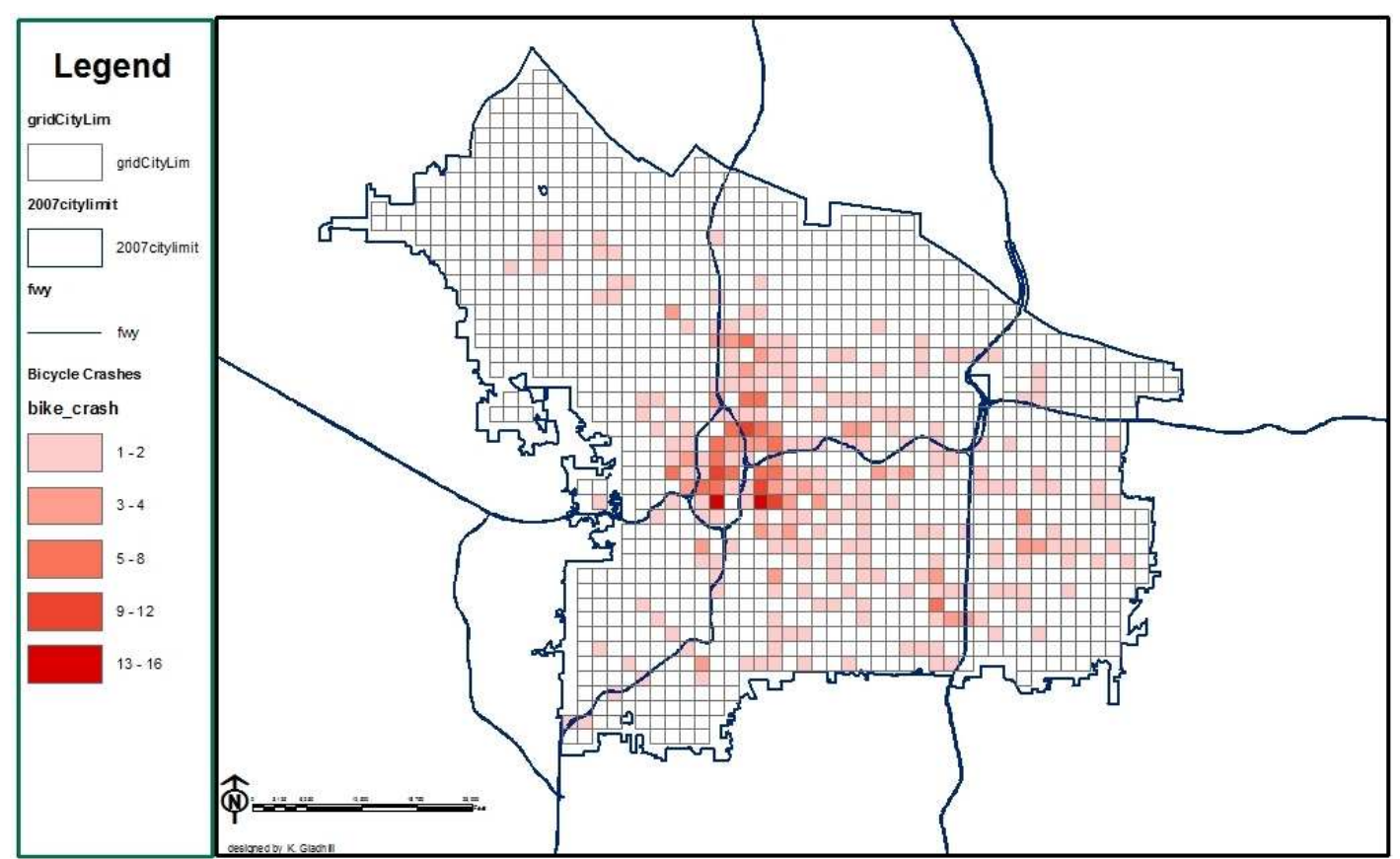

Figure 122005 - 2007 Bicycle Crash Spatial Map

Page 33 
Vehicle crashes are shown in Figure 13. PDO crashes (see Figure 14), show essentially the same distribution as total vehicle crashes, which is expected considering that PDO were more than $60 \%$ of the vehicle crashes. Figure 15 shows all fatal and injury crashes, still in a similar distribution to total vehicle crashes. Fatal crashes were rarer, as seen in Figure 16. Adding injury level A crashes in Figure 17 starts to again make major corridors discernible.

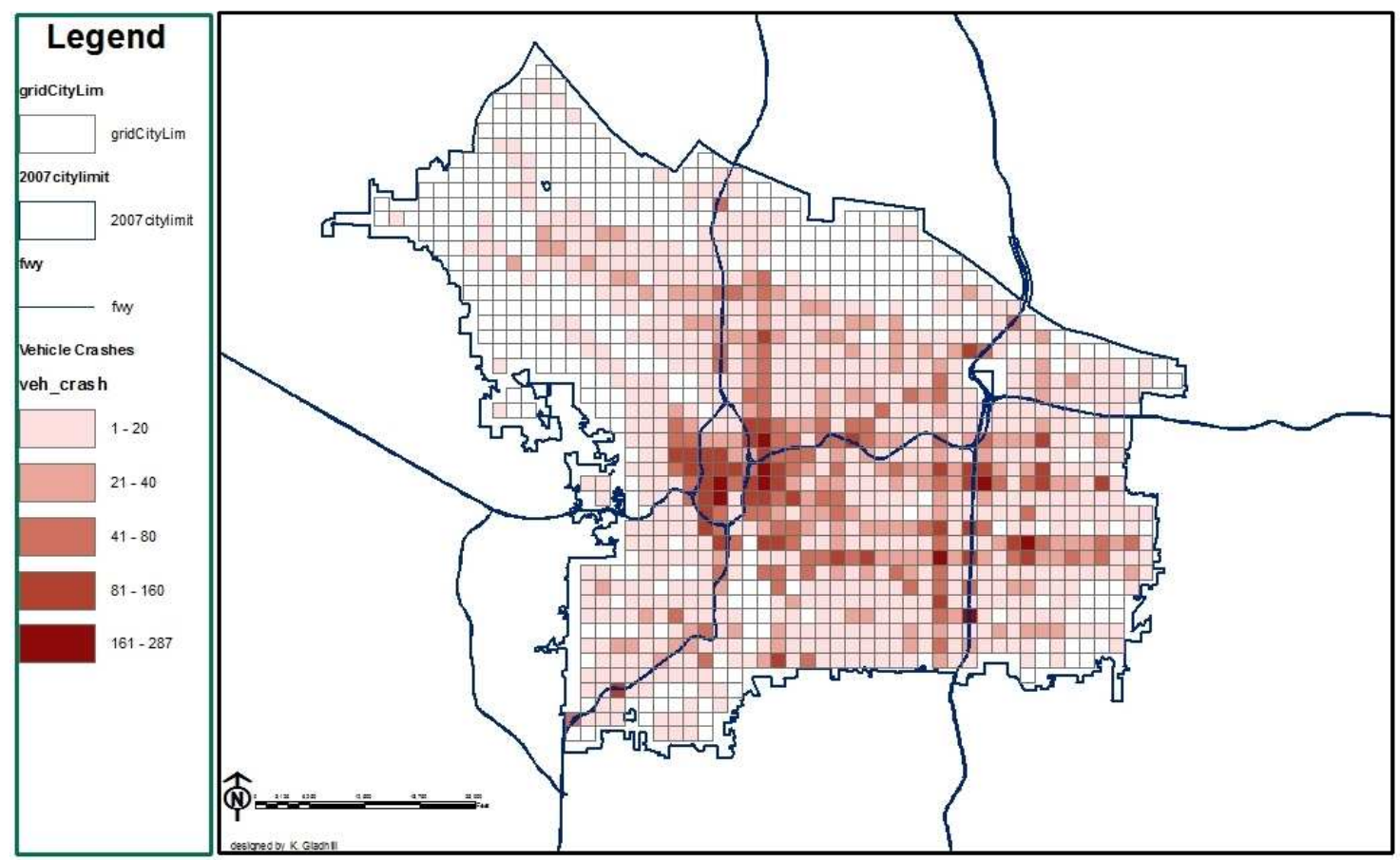

Figure 132005 - 2007 Vehicle Crashes 


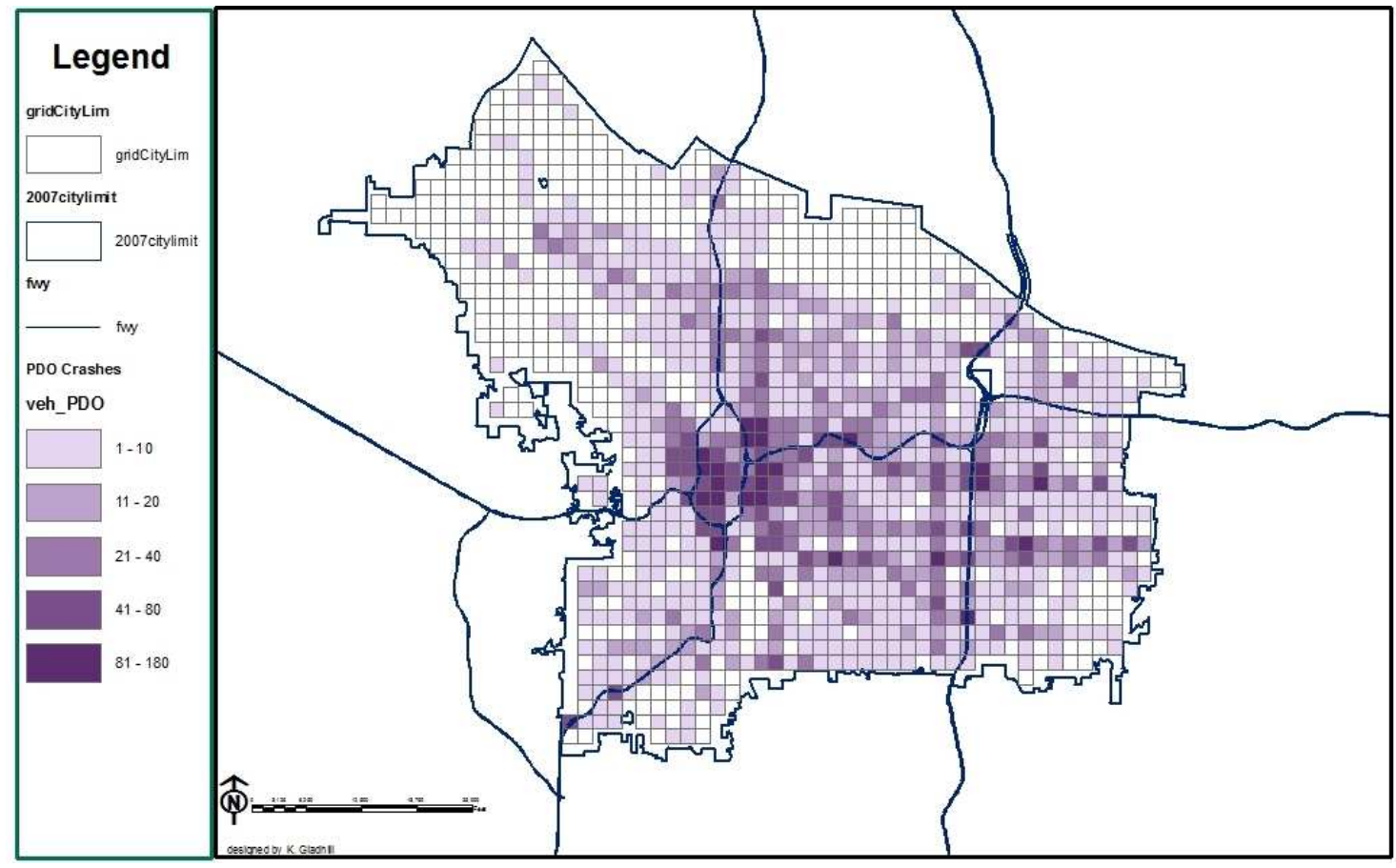

Figure 142005 - 2007 Vehicle Crashes, PDO Severity

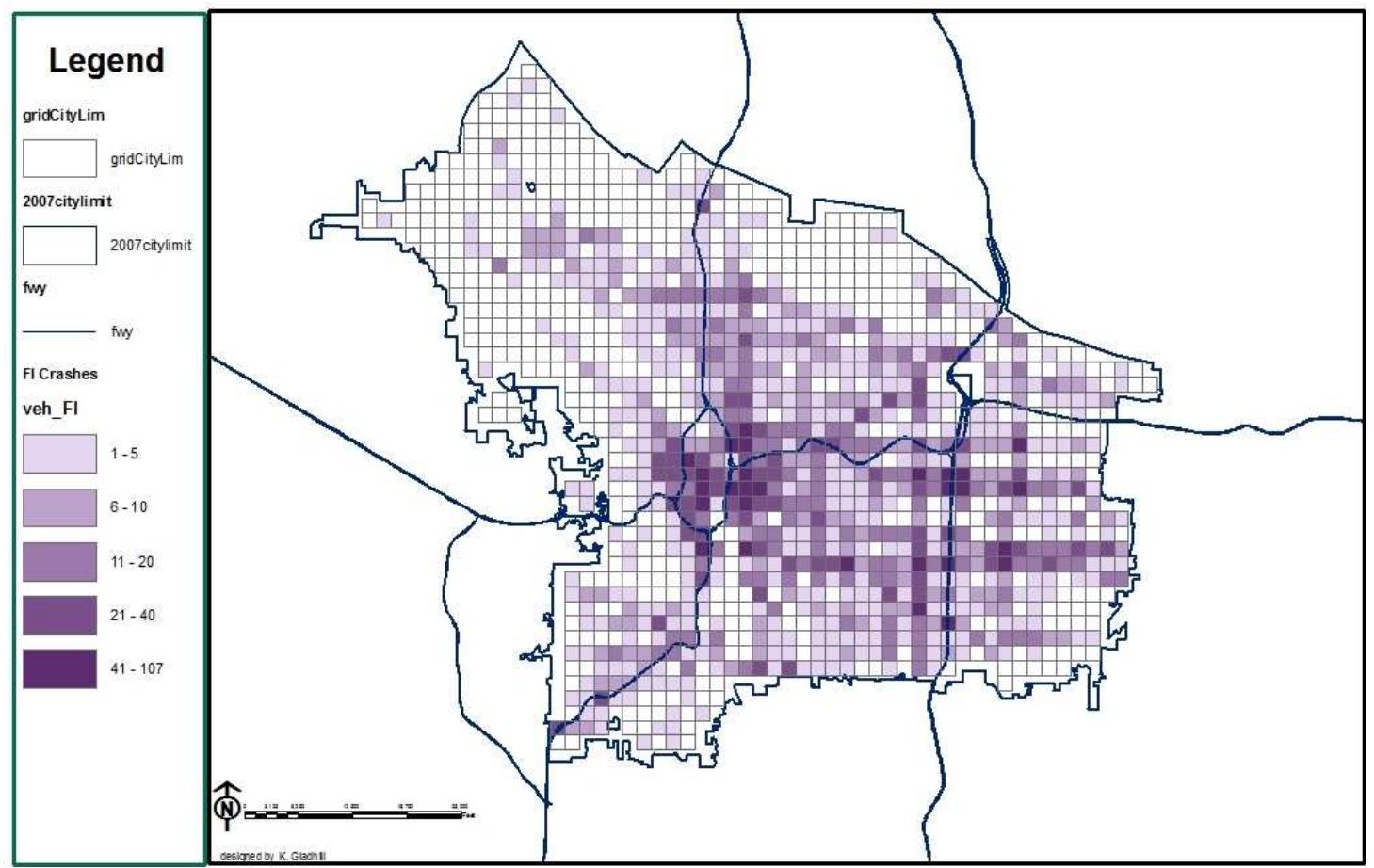

Figure 152005 - 2007 Vehicle Crashes, FI Severity

Page 35 


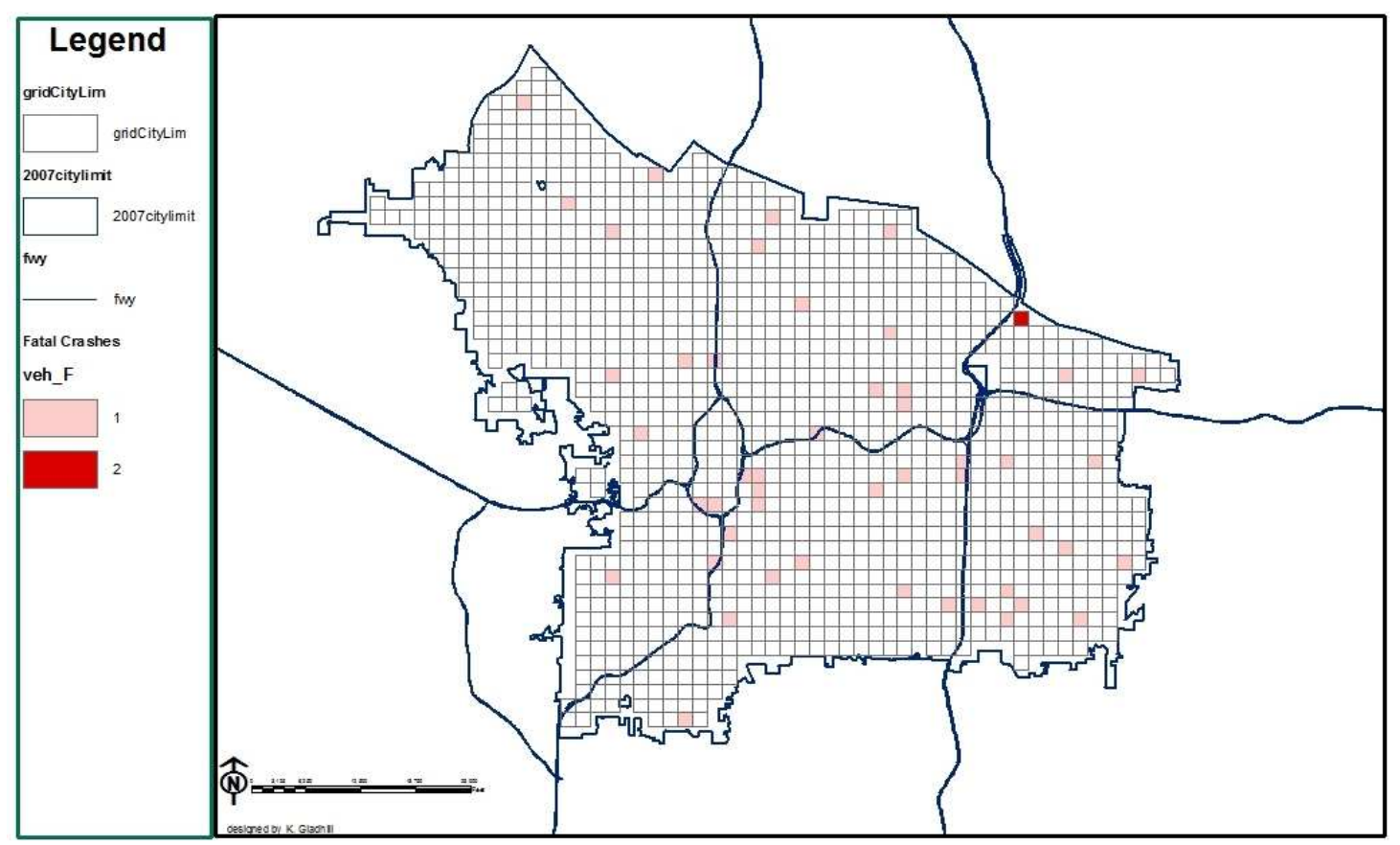

Figure 162005 - 2007 Vehicle Crashes, Fatal

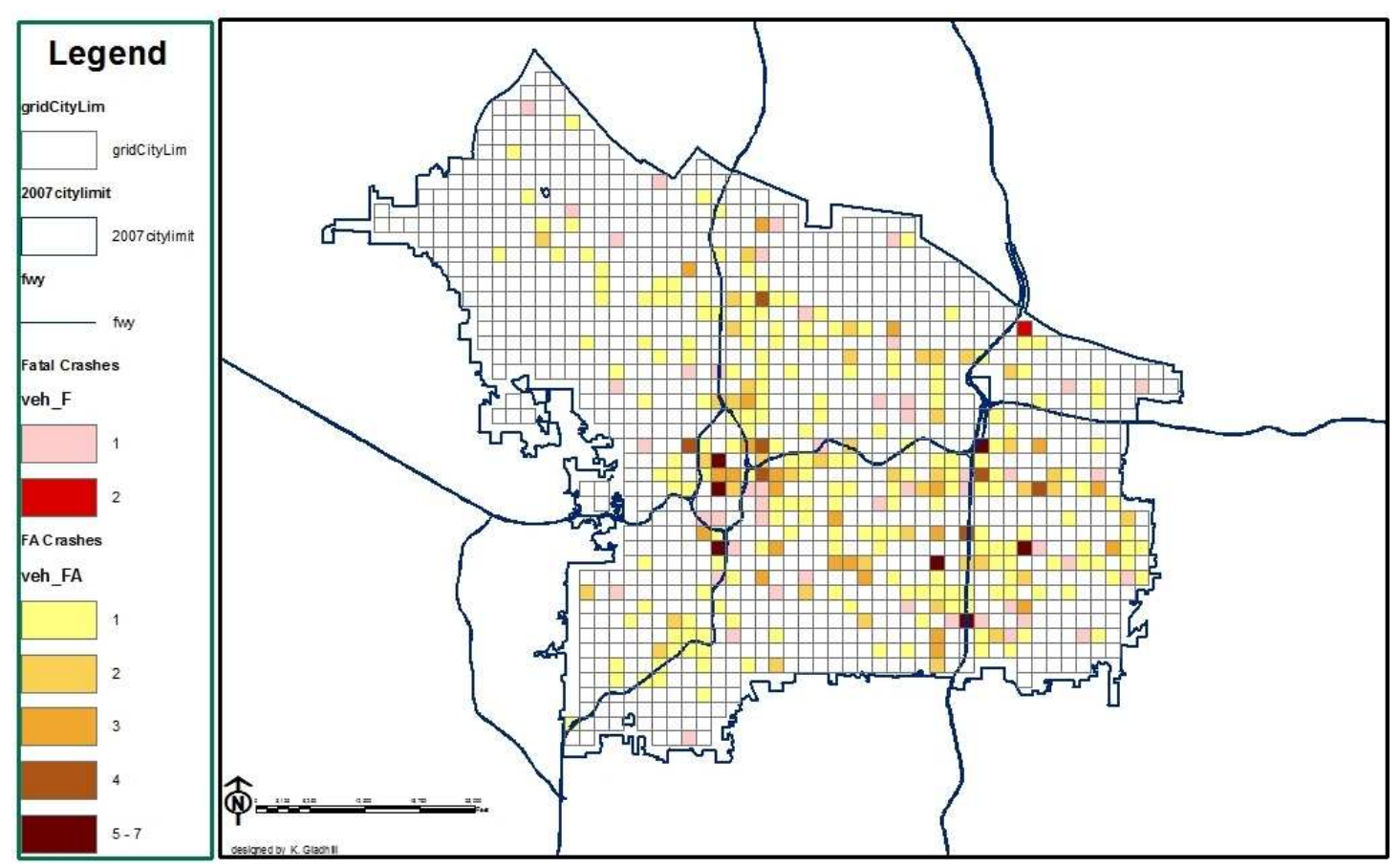

Figure 172005 - 2007 Vehicle Crashes, Fatal and Injury A Severity

Page 36 


\subsection{Exposure}

Higher exposure on the transportation network typically increases crash risk. This study considered exposure factors for vehicles, not for pedestrians nor bicycles. Exposure data were represented by volume to capacity ratios, VMT (vehicle miles traveled), average free speed, and presence of schools. Volume to capacity ratio $(\mathrm{v} / \mathrm{c})$ values within a spatial grid cell were averaged for each grid cell, see Figure 18. Downtown did not have the highest v/c ratios. Instead, some particular arterial corridors, such as Powell Boulevard in the southeast and Barbur to the southwest can be seen in the v/c spatial map. Note that some spatial grid cells had no $\mathrm{v} / \mathrm{c}$ data. These cells were eliminated from the modeling dataset, reducing the cells for modeling consideration from 1284 to 928 total cells

Average free speed is shown in Figure 19, with major arterials having higher speeds and the highest average free speeds in outlying areas. These average free speeds are based on posted speeds, and are inputs to the travel demand model rather than modeled values. 


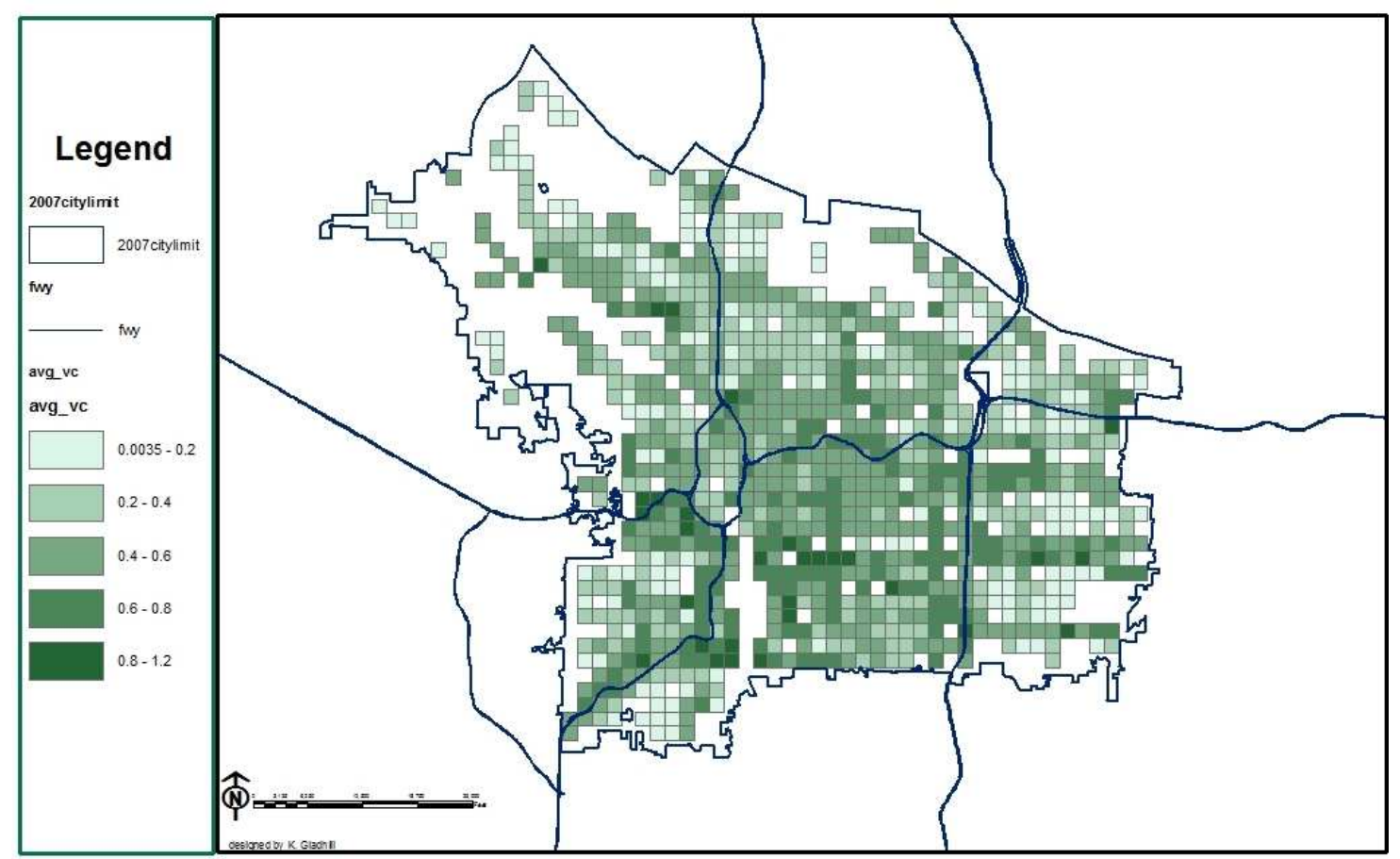

Figure 182005 Average of Volume to Capacity Ratios within a Spatial Grid Cell

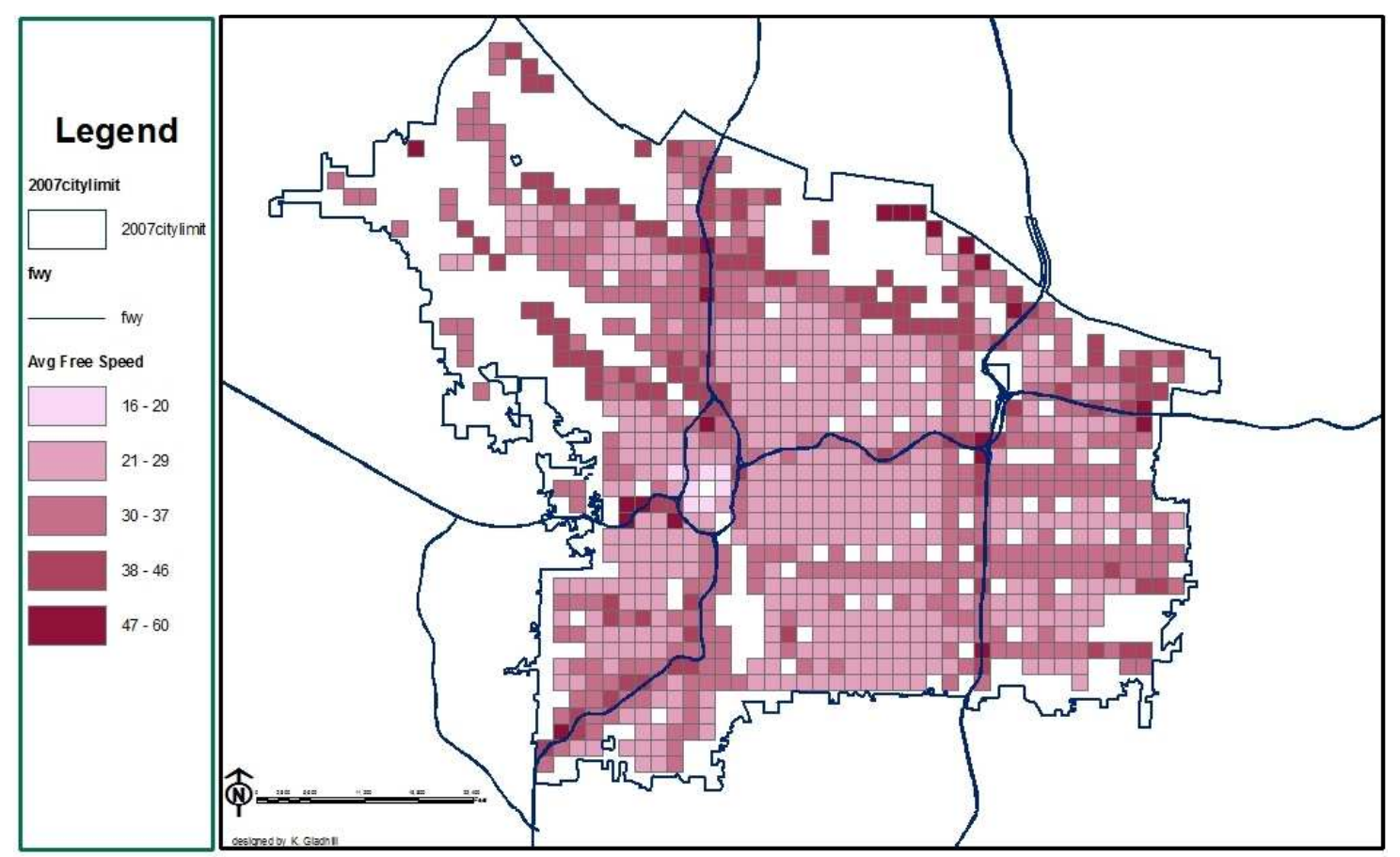

Figure 192005 Average Free Speed

Page 38 


\subsection{Connectivity}

Connectivity and urban form were looked at using several factors One was the four level street network scale developed by Rifaat and Tay (4) of loops and lollipops, mixed, warped parallel, or grid (see Figures 1 through 4), referred to as the "street network" factor in this study, Some spatial grid cells did not have a discernible type, and were assigned a value of zero. These cells were also removed from the modeling dataset, taking the set down to 792 spatial cells, still a considerable sample size. Figure 22 is a histogram of the frequency that the four different street network values were assigned.

The percentage of intersections in each spatial grid cell that were four leg intersections was also calculated. This would be $100 \%$ for full grid street layout, decreasing to a lower percentage as the street network becomes less grid like. Zero could be achieved if only 3 leg intersections are designed into a loops and lollipops style of development. The percentage four leg intersections could be calculated, and was thus less subjective than applying the street network scale in Figure 20. Percentage four leg intersections also has continuous rather than discrete numeric values, generally better for modeling.

The percentage four leg intersections correlated well with the Rifaat and Tay street network scales visually, see Figure 20 and Figure 21 . Both factors were used in modeling.

Page 39 


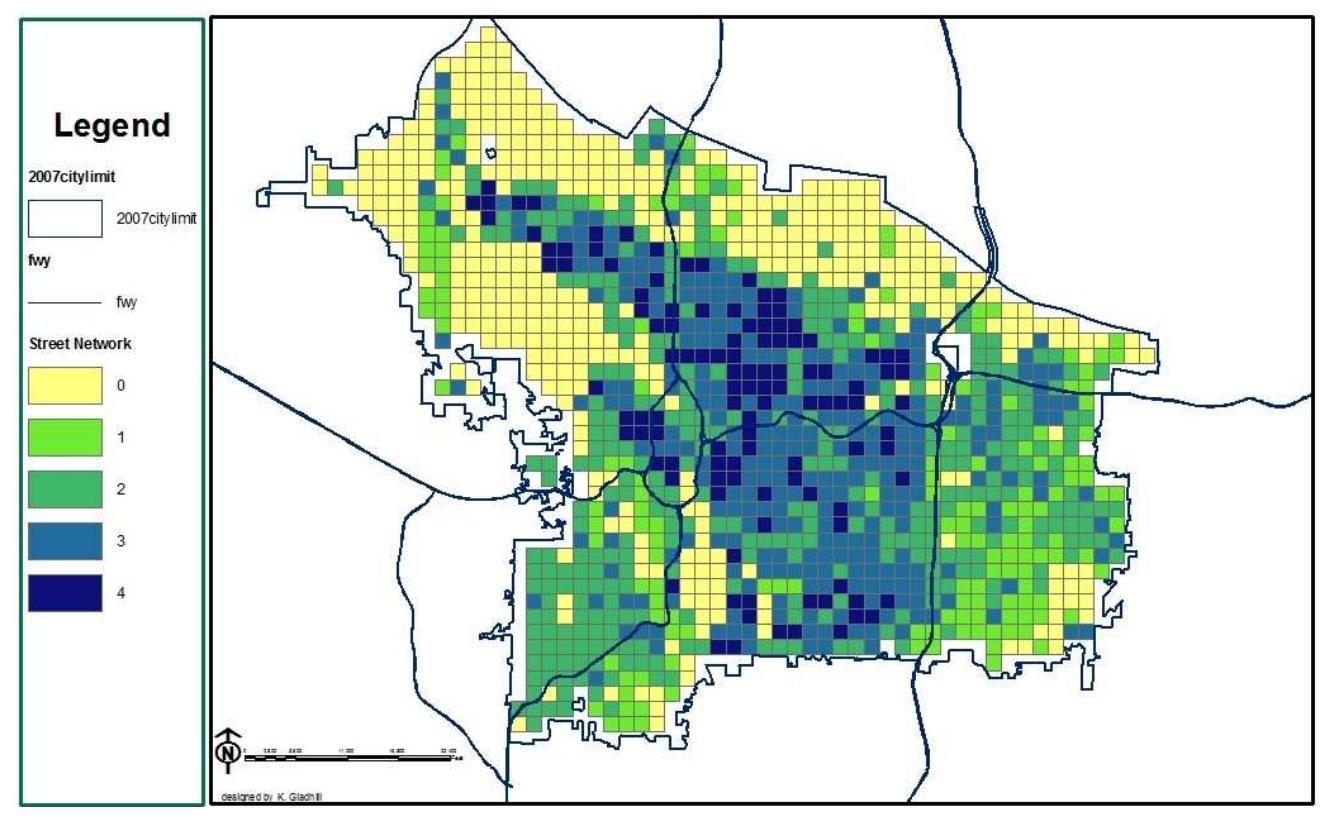

Figure 20 Street Network Value Assignments

$1=$ Loops and Lollipops $\quad 2=$ Warped Parallel $\quad 3=$ Mixed

$4=$ Grid

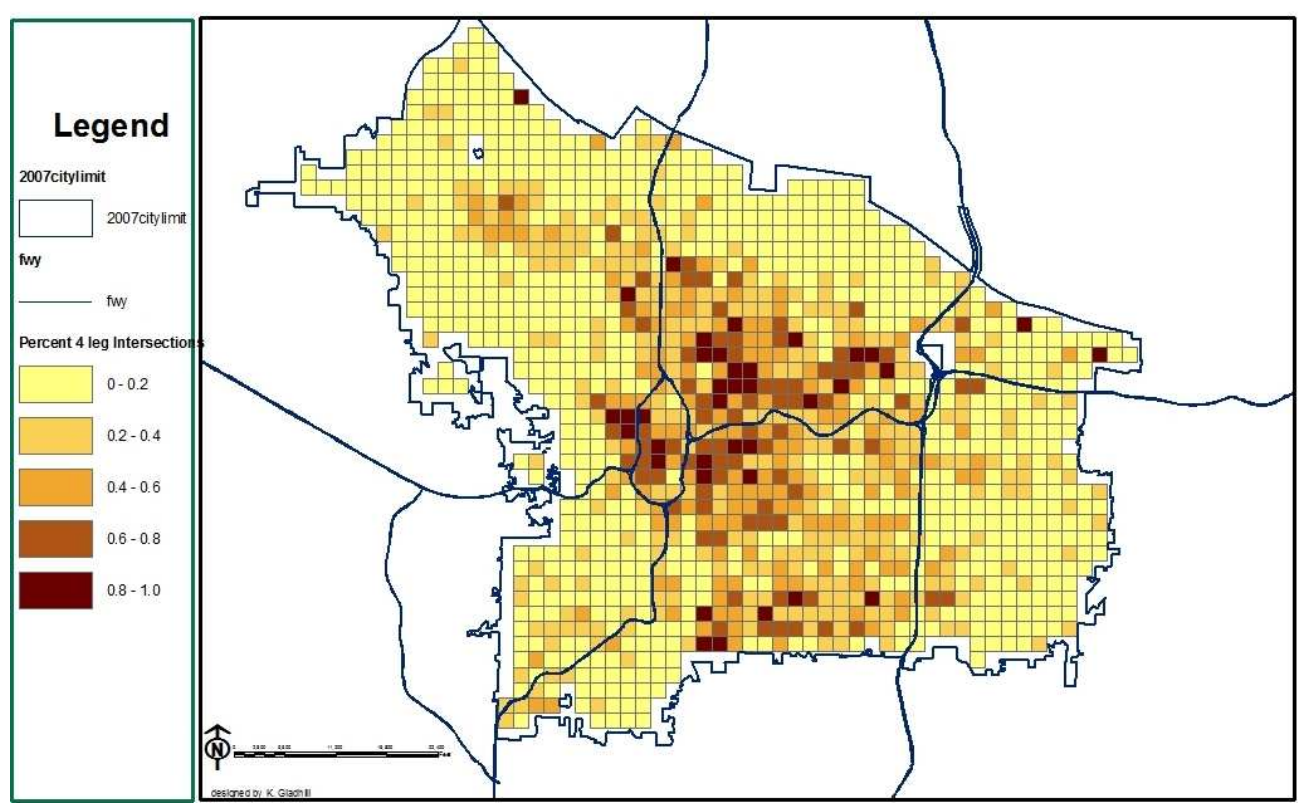

Figure 21 Percentage of Intersections which are Four Leg Intersections

Page 40 


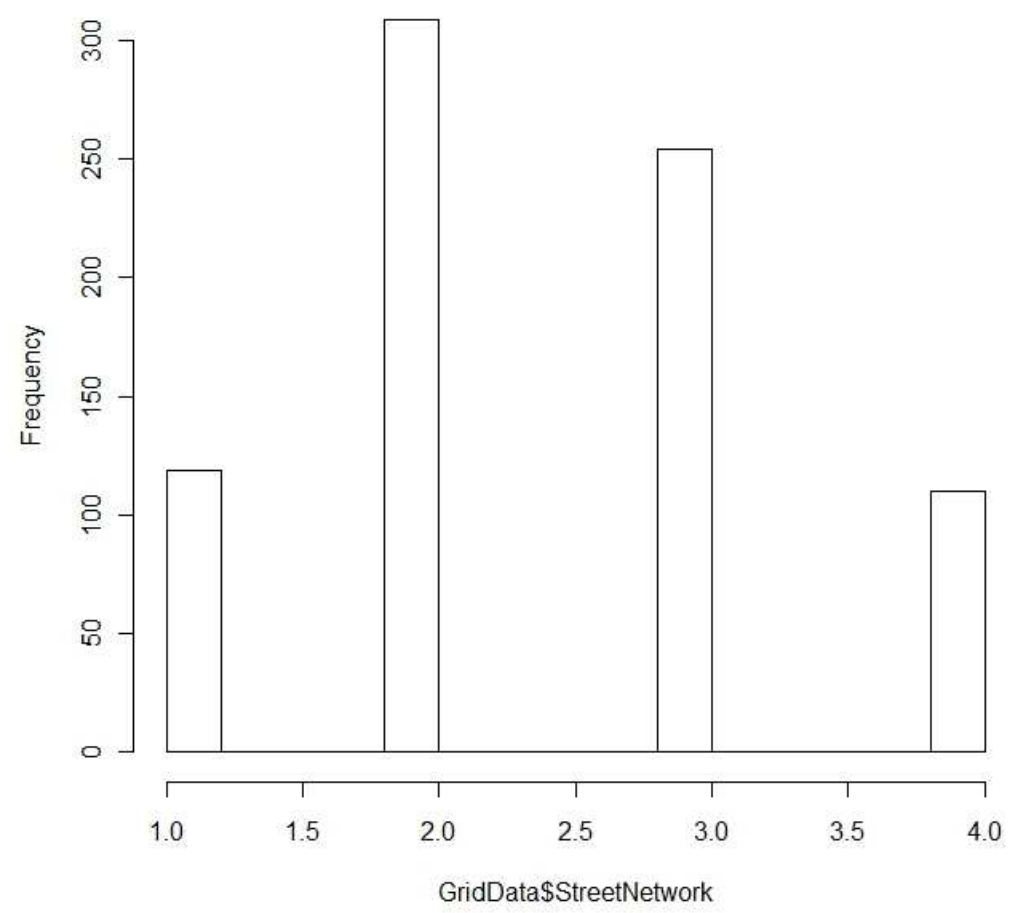

Figure 22 Histogram of Street Network Values

$1=$ Loops and Lollipops $\quad 2=$ Warped Parallel $\quad 3=$ Mixed $\quad 4=$ Grid

Modeling also included total major arterial length as a factor, since it did not correlate to other street and intersection factors, see Appendix E, Figure E-1.

\subsection{Transit Accessibility}

Several factors were considered to represent transit accessibility. As can be seen in Figure 23, transit stops are widespread throughout the Portland city limits along major arterial routes and concentrated in the downtown area. Outlying areas have sparse or no transit service. This corresponds to population Page 41 
and housing density, shown in later figures, indicating a well planned transit system which has more service in areas of greater demand.

Total boardings were also considered, as well as route length.

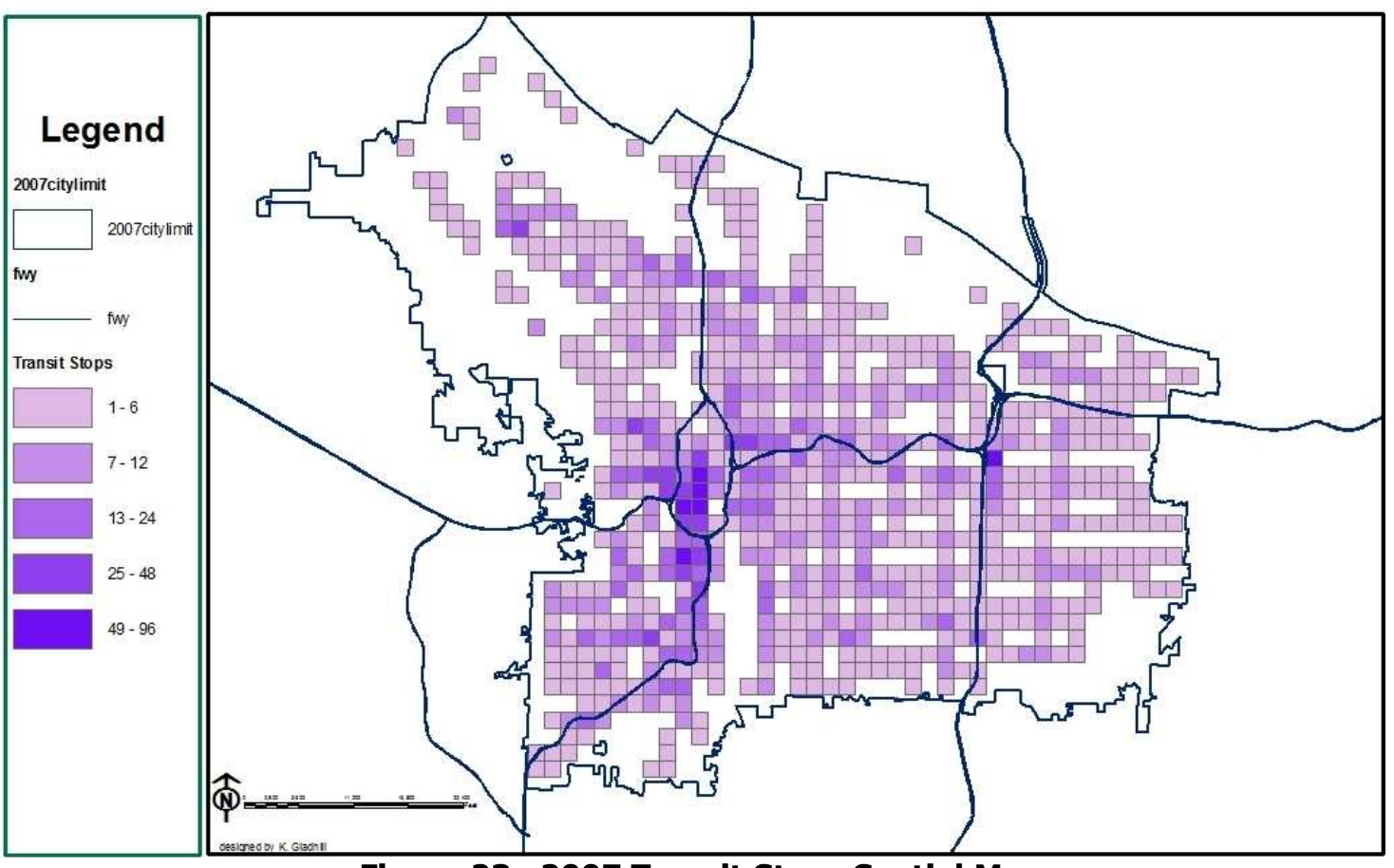

Figure 232007 Transit Stops Spatial Map

\subsection{Demographics}

The highest population was concentrated in the downtown area, (see Figure 24). There was a large area with high population density east of the Willamette River, and sparse population in outlying areas. Dwelling units (Figure 25) and households showed the same distribution. 


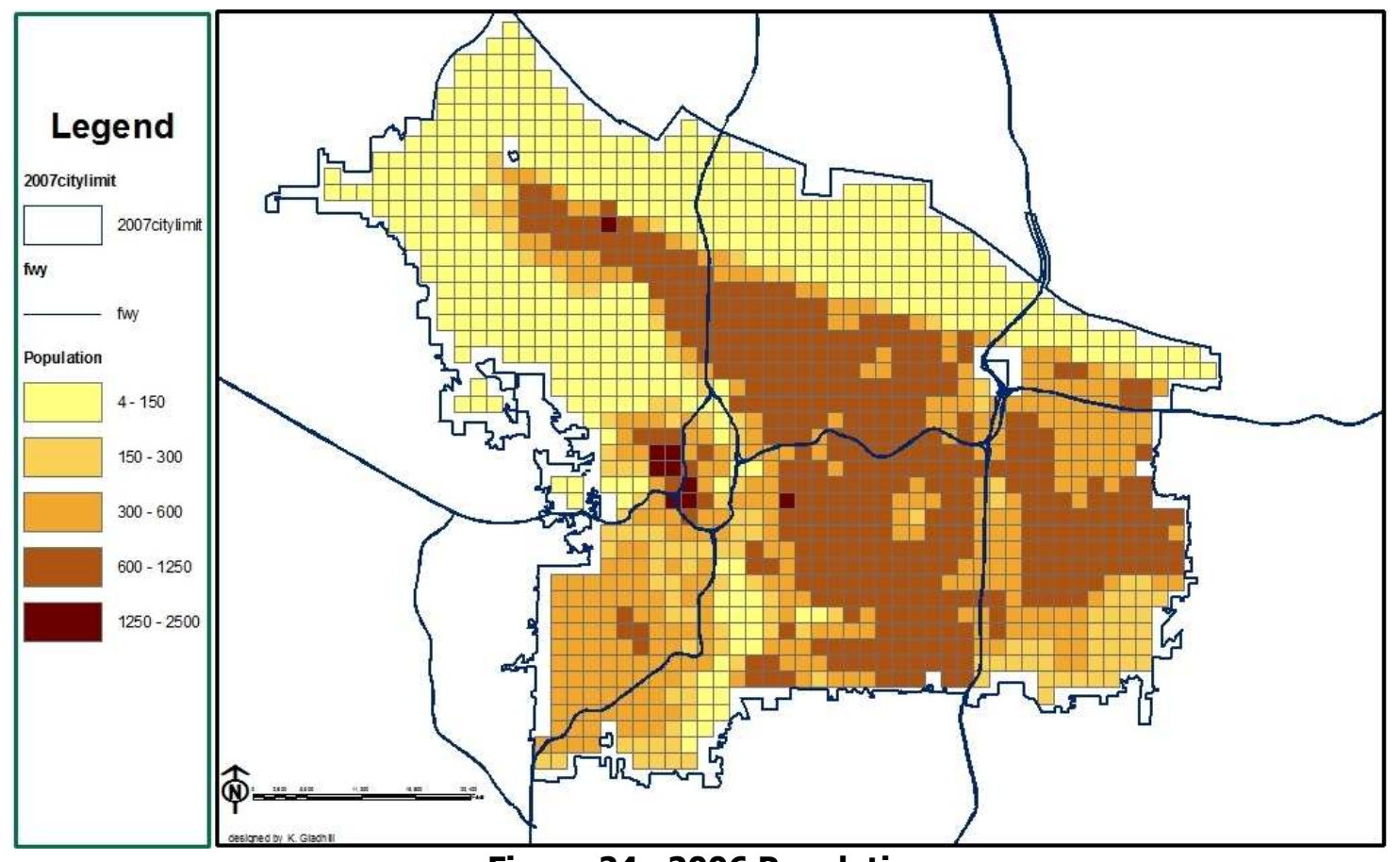

Figure 242006 Population

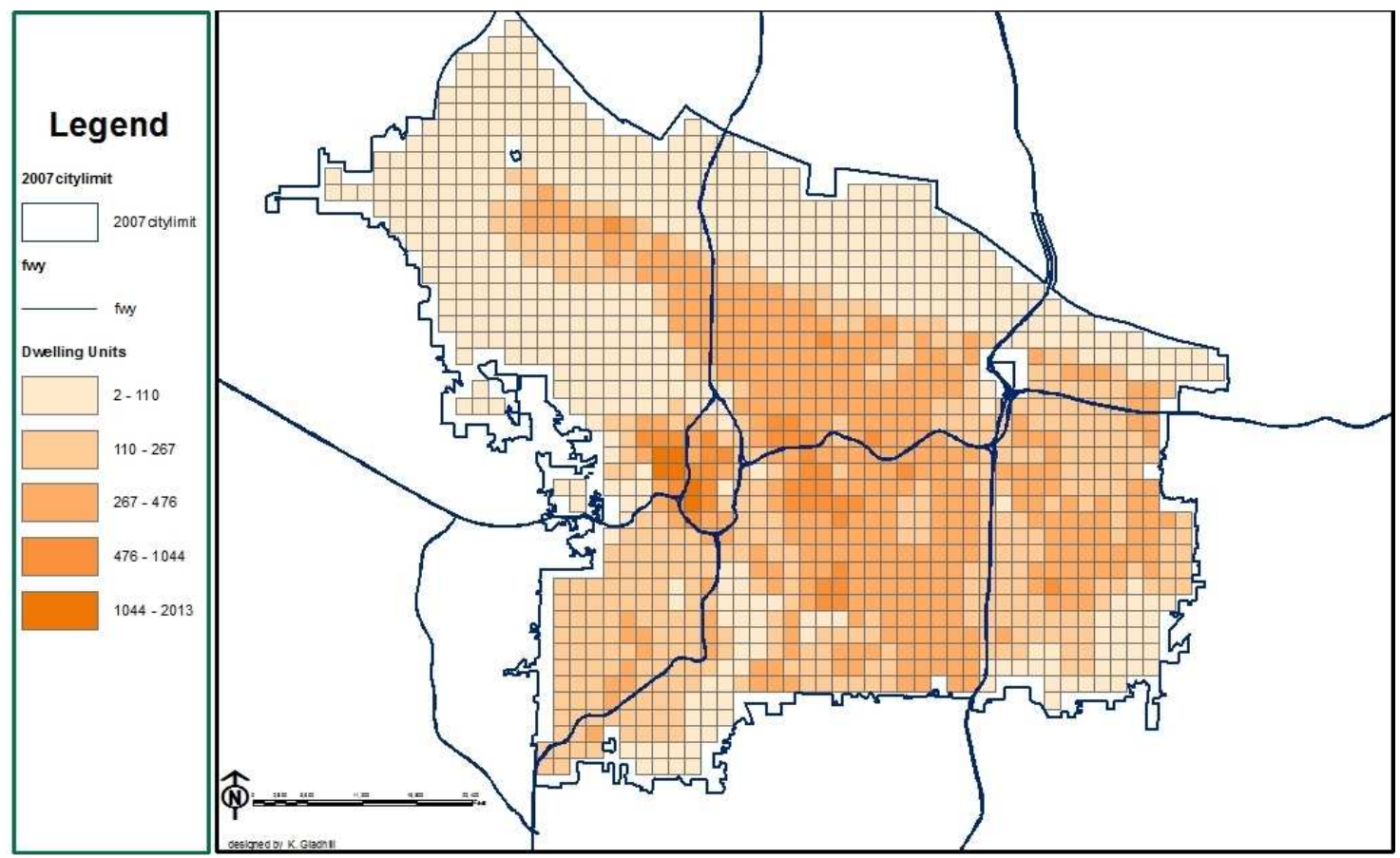

Figure 252006 Dwelling Units Spatial Map

Page 43 


\subsection{Origins and Destinations}

Employment (Figure 26) and the number of business establishments

(Figure 27) were included to represent origins and destinations, since there is travel to get to and from work, as well as to patronize businesses of all sorts. Employment, or the number of employees, was highly concentrated around the downtown area, with some satellite areas. The number of business establishments also was concentrated heavily in the downtown area, but had a more diverse spread throughout the city, corresponding to the densely populated areas seen in Figure 24.

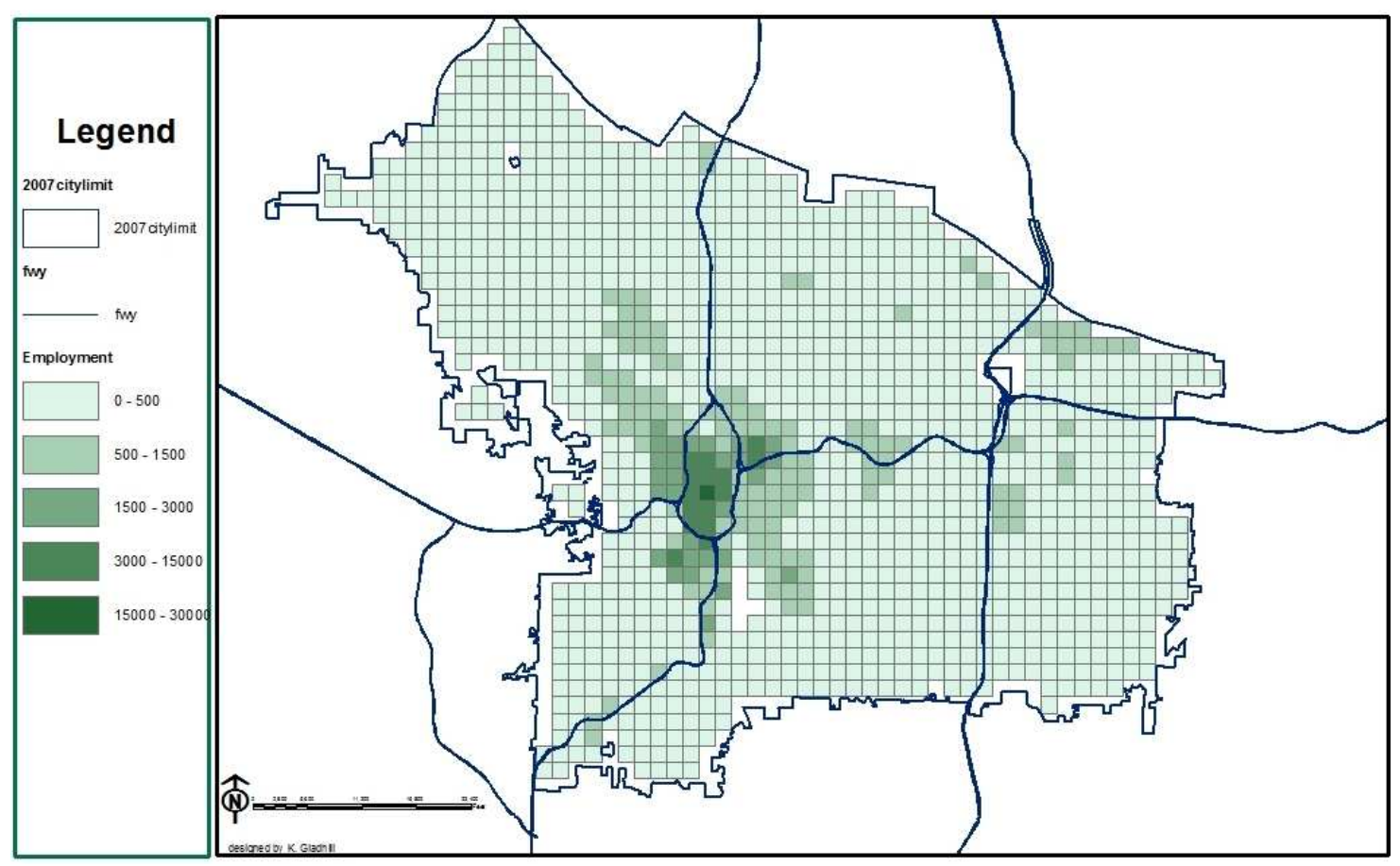

Figure 262005 Employment Spatial Map 


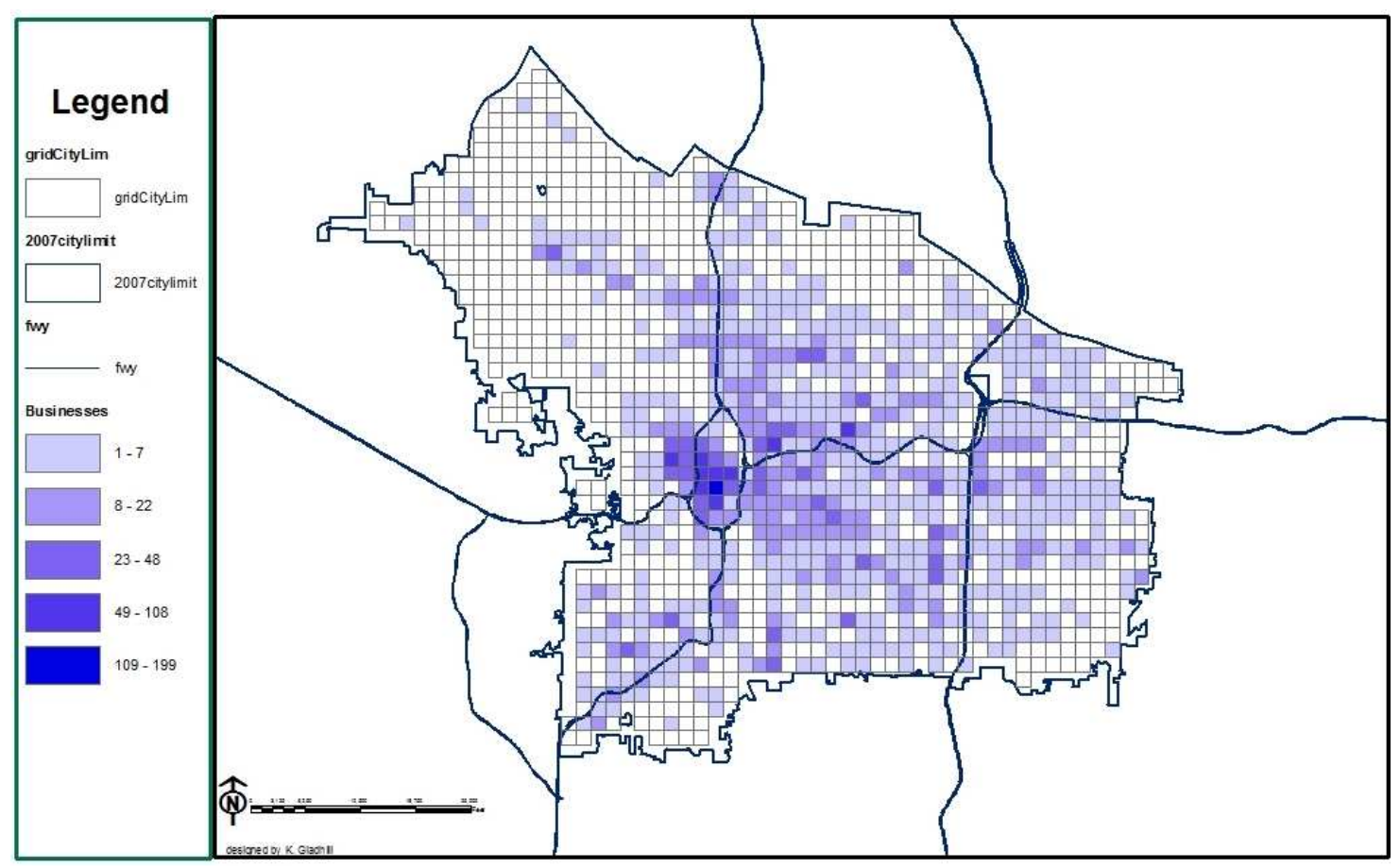

Figure 272010 Business Spatial Map

Page 45 


\subsection{MODEL DEVELOPMENT AND RESULTS}

\subsection{Development}

\subsubsection{Summary of Input Data}

Spatial grid cells with street length less than 3,300 feet, which would correspond to twice the length of the side of a cell, were eliminated as a lower bound on street length in a cell for urban form to be evident. Cells with no volume model data (VMT, v/c) were also eliminated. This left a dataset with 928 spatial grid cells for modeling. A further reduction was made to eliminate cells which had street network of zero, indicating that I had been unable to determine which of the four Rifaat and Tay street network categories it corresponded to. With this final reduction, the dataset was 792 spatial grid cells. A summary of the model input data for 792 spatial grid cells can be found in Table 6 . Minimum, mean, maximum, standard deviation, and totals are shown.

\subsubsection{Selection of Independent Variables}

Many of the independent variables were correlated. Using Pearson's correlation and qualitative analysis, these were reviewed and reduced to a smaller set of factors to be used for modeling (see Appendix E). For example, since population was highly correlated with household and dwelling units, population was chosen to use in the modeling, representing all three 
demographic factors. Bus stops and routes were the majority of the transit stops and routes, and all highly correlated, so there was no need to duplicate these factors by including them separately: only the transit stops factor was left in the model. Transit boardings and alightings, (ons and offs), were highly correlated; the sum of ons and offs was included in the model building. Street length and intersection factors showed cross correlation. Street network street length, major arterial length, percent four leg intersections, total intersections, and block size represented connectivity factors. 
Table 6 Modeling Input Data Summary

\begin{tabular}{|c|c|c|c|c|c|c|}
\hline & Min. & Mean & Max. & Median & std dev & TOTAL \\
\hline \multicolumn{7}{|l|}{ CRASH DATA } \\
\hline Vehicle Crashes & 0 & 24.82 & 287 & 13 & 34.56 & 19658 \\
\hline Pedestrian Crashes & 0 & 0.5947 & 9 & 0 & 1.222 & 471 \\
\hline Bicycle Crashes & 0 & 0.649 & 16 & 0 & 1.527 & 514 \\
\hline Angle Crash Type & 0 & 8.963 & 139 & 4 & 15.48 & 7099 \\
\hline Turn Crash Type & 0 & 1.726 & 20 & 0 & 2.92 & 1367 \\
\hline Straight Crash Type & 0 & 2.518 & 44 & 1 & 4.62 & 1994 \\
\hline Stop Crash Type & 0 & 7.304 & 70 & 3 & 11.16 & 5785 \\
\hline Fatal Crashes & 0 & 0.05051 & 1 & 0 & 0.219 & 40 \\
\hline Fatal, Injury A Crashes & 0 & 0.5896 & 7 & 0 & 1.01 & 467 \\
\hline Injury Crashes & 0 & 9.068 & 107 & 5 & 12.71 & 7182 \\
\hline Fatal \& Injury Crashes & 0 & 9.119 & 107 & 5 & 12.74 & 7222 \\
\hline PDO Crashes & 0 & 15.7 & 180 & 8 & 22.48 & 12436 \\
\hline \multicolumn{7}{|l|}{ EXPOSURE } \\
\hline VMT & 2.392 & 2045.78 & 14623.3 & 10328 & 2680 & $1.62 \mathrm{E}+6$ \\
\hline $\mathrm{v} / \mathrm{c}$ & 0.0035 & 0.438 & 1.215 & 0.4547 & 0.201 & \\
\hline Avg Free Speed & 15.5 & 30.23 & 60 & 29 & 5.83 & \\
\hline schools count & 0 & 0.2803 & 4 & 0 & 0.586 & 222 \\
\hline \multicolumn{7}{|l|}{ CONNECTIVITY } \\
\hline Street Network scale & 1 & 2.448 & 4 & 2 & 0.909 & \\
\hline intersections & 0 & 23.67 & 81 & 22 & 11.34 & 18750 \\
\hline fourLegPct & 0 & 0.311 & 1 & 0.2697 & 0.229 & \\
\hline nonFwy Street & 3649 & 12682 & 28697 & 12481 & 3789 & $10 \mathrm{E}+6$ \\
\hline Major Arterial length & 0 & 1535.7 & 18547.6 & 212.8 & 2471 & $1.22 \mathrm{E}+6$ \\
\hline block Size & 9 & 61.39 & 325 & 55 & 33.09 & \\
\hline \multicolumn{7}{|l|}{ ACCESSIBILITY } \\
\hline Transit Stops & 0 & 5.987 & 87 & 4 & 8.09 & 4742 \\
\hline On + Off & 0 & 173305 & 8715715 & 27212 & 592000 & $1.4 \mathrm{E}+08$ \\
\hline \multicolumn{7}{|l|}{ DEMOGRAPHIC } \\
\hline Population & 4.1 & 559.3 & 2475.7 & 567.7 & 303 & 442939 \\
\hline \multicolumn{7}{|c|}{ ORIGINS AND DESTINATIONS } \\
\hline Employment & 0.39 & 149.25 & 346.32 & 157.24 & 936 & 319731 \\
\hline Business density & 0 & 5.77 & 108 & 2 & 10.73 & 4570 \\
\hline
\end{tabular}

Page 48 


\subsubsection{Model Building}

Since crashes are essentially a failure event, crash data do not follow a normal distribution. Poisson modeling can be considered, but the likelihood of many zero values recommends that a check be made for over dispersion. Poisson modeling with the study data confirmed that the data were over dispersed, so negative binomial regression would be an appropriate model (15), of the form:

$$
\begin{aligned}
\mathrm{N}_{\text {crash }}=\exp \left(\mathrm{a}+\mathrm{b}^{*} \mathrm{x}_{\mathrm{b}}+\mathrm{c}^{*} \mathrm{x}_{\mathrm{c}}+\ldots+\mathrm{n} * \mathrm{x}_{\mathrm{n}}\right) \\
\text { where } \mathrm{N}_{\text {crash }} \quad=\text { number of crashes } \\
\begin{aligned}
\text { a through } \mathrm{n} & =\text { coefficients } \\
\mathrm{x}_{i} & =\text { factor } i \text { value }
\end{aligned}
\end{aligned}
$$

VMT provided a better fit as $\log (\mathrm{VMT})$, equivalent to putting VMT before the exponent in the formula, which was consistent with crash models that are have volume outside the exponential term:

$$
\begin{array}{rl}
\mathrm{N}_{\text {crash }}=\mathrm{VMT} & * \exp \left(\mathrm{a}+\mathrm{b}^{*} \mathrm{x}_{\mathrm{b}}+\mathrm{c}^{*} \mathrm{x}_{\mathrm{c}}+\ldots+\mathrm{n} * \mathrm{x}_{\mathrm{n}}\right) \\
\text { where } \mathrm{N}_{\text {crash }} \quad=\text { number of crashes } \\
\qquad \begin{aligned}
\mathrm{VMT} & =\text { vehicle miles traveled } \\
\text { a through } \mathrm{n} & =\text { coefficients } \\
\mathrm{x}_{i} & =\text { factor } i \text { value }
\end{aligned}
\end{array}
$$


The street network factor was included in the negative binomial models as a factor, having discrete values of:

$$
\begin{aligned}
& 1=\text { loops and lollipops } \\
& 2=\text { mixed } \\
& 3=\text { warped parallel, and } \\
& 4=\text { grid street layout }
\end{aligned}
$$

which compared each of the other levels to level $1=$ loops and lollipops.

Negative binomial models were developed for vehicle crashes, pedestrian crashes, and bicycle crashes separately. Preliminary analysis had indicated that the top four crash types were angle crashes, turning crashes, straight, and stopped vehicle crashes. Separate models were developed for each of these crash types.

Separate models were also developed for levels of crash severity. Modeling was unsuccessful on fatalities alone due to the small number of non-zero data points. Crash severity was looked at for several groupings: "FA" grouped fatal and injury level A (incapacitating) together, the most severe crash injuries; "FI" grouped fatal and all three injury levels ( $\mathrm{A}$ = incapacitating, $B=$ non-incapacitating, $C=$ possible injury) together; "I" grouped the three injury levels, and PDO (property damage only crashes).

Negative binomial regression models were developed in a step-wise 
fashion by adding one factor at a time using the cumulative regression method described by Banfro and Hauer (16) to decide whether the addition of a factor improved the model. This method involved inspection of the regression diagnostic plots (cumulative residual plots) for each model as the new factor was added. The standard deviation for the 2 sigma limits was calculated as follows:

$$
\begin{aligned}
& \sigma^{* 2}=\sigma^{2}(n)\left[1-\sigma^{2}(n) / \sigma^{2}(N)\right] \\
& \text { where } \begin{aligned}
\sigma^{*} & =\text { standard deviation, } \\
\sigma(\mathrm{n}) & =\text { sigma for the current value } \\
\sigma(\mathrm{N}) & =\text { sigma for all values }
\end{aligned}
\end{aligned}
$$

The cumulative residual plots were judged as to whether the cumulative residuals were within the \pm 2 sigma limits, the 2 sigma limits were getting narrower, the cumulative residuals were centered around zero, and the final cumulative of residuals was closer to zero, since the cumulative residuals should theoretically sum to zero. If the cumulative regressions looked better with the second factor, the factor was kept in the model. If not, the factor was dropped from the model. Figure 28 shows that adding the block size factor into the vehicle crash model improves the cumulative regression. $\mathrm{R}$ code for developing these CURE plots is included in the Appendix.

Other model fit plots were also examined. As seen in Figure 29, the standard deviation residuals Q-Q plot should be approaching a straight line.

Finally, a negative binomial regression was also run with all the modeling 
factors, followed by a case with the significant factors from the all factors model. The model from the "add a factor" approach was then compared to the "all factors" and "all significant factors" models by comparing cumulative residual charts for non-freeway street length, and the best one selected for the results.
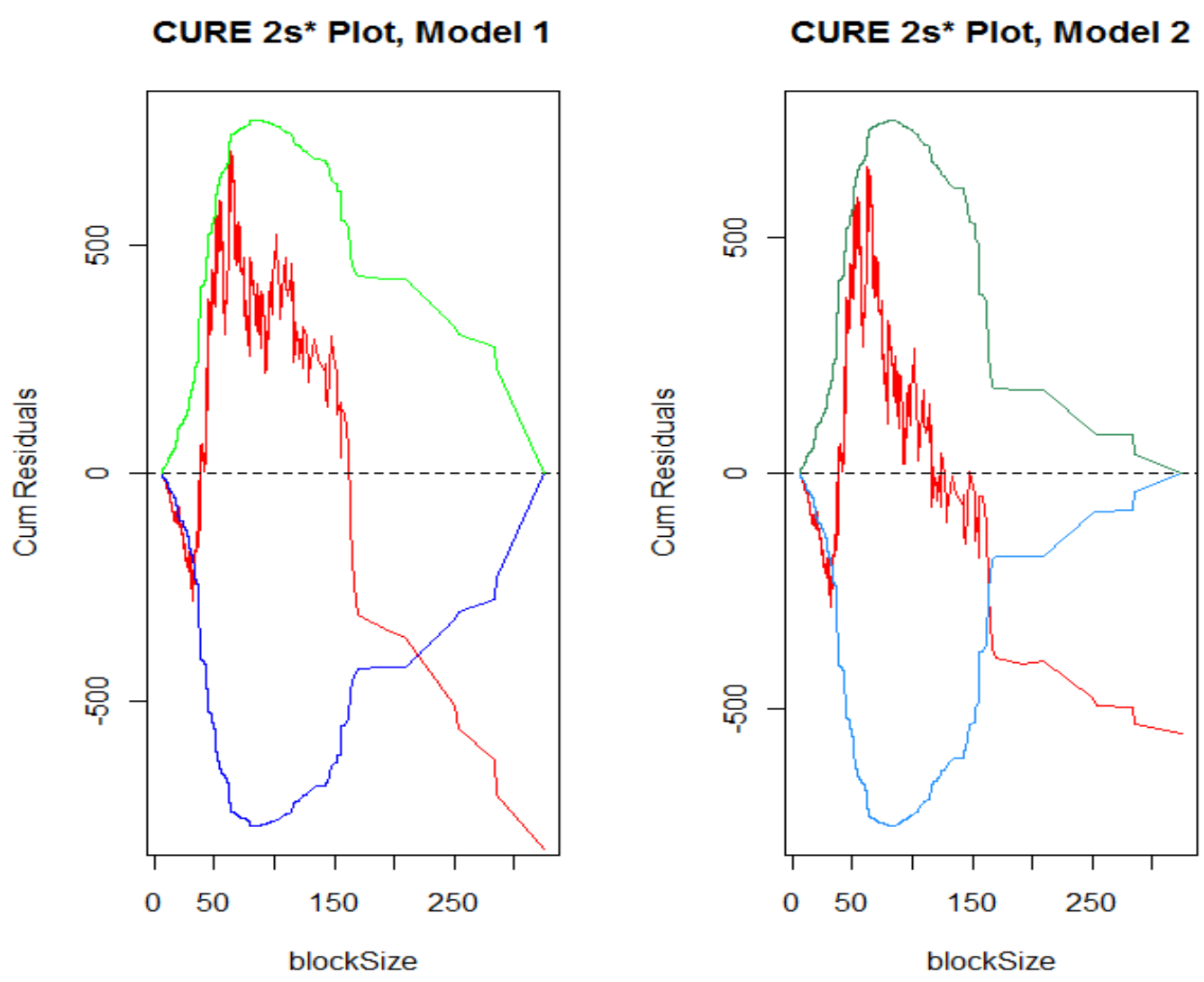

Figure 28 Cumulative Residual CURE 2 sigma Plots for Comparison 

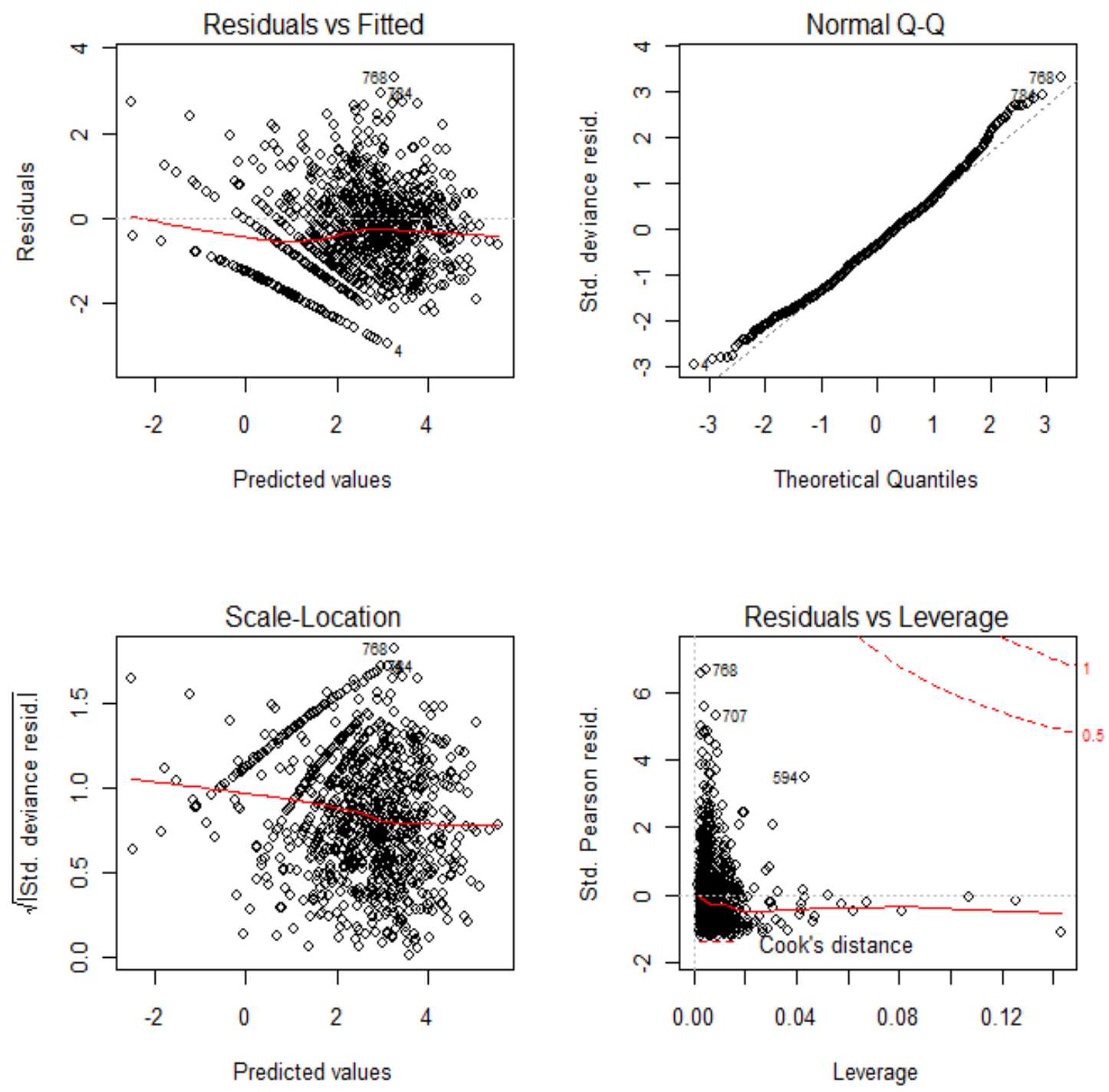

Figure 29 Plots for Negative Binomial Regression Fit

Page 53 


\subsection{Model Results}

Table 7 shows goodness of fit for the negative binomial modeling results. All models had the same sample size, 792 spatial grid cells. Note that the models have better fit where there are more crash data: vehicle crashes, angle and stopped vehicle crashes; all injury crashes, and PDO crashes. The standard error is low, and estimated R2 values look good for crash modeling

Table 7 Negative Binomial Modeling Goodness of Fit

\begin{tabular}{|c|c|c|c|c|c|c|c|c|c|}
\hline & 宸 & $\begin{array}{l}\underset{\mathbb{Z}}{\mathbb{E}} \\
\underset{+}{+}\end{array}$ & $\begin{array}{l}\frac{1}{2} \\
\frac{2}{0} \\
\frac{D}{\omega}\end{array}$ & 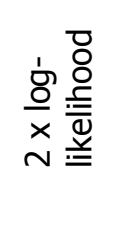 & 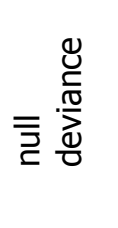 & 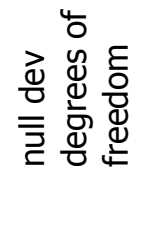 & 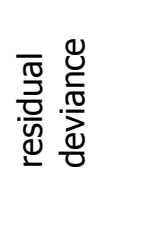 & 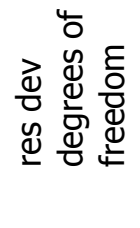 & 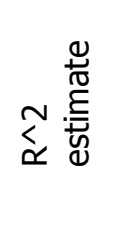 \\
\hline \multicolumn{10}{|c|}{ Crash Group } \\
\hline Vehicle & 5854 & 2.037 & 0.119 & -5920 & 2298 & 791 & 875 & 776 & 0.62 \\
\hline Pedestrian & 1393 & 1.43 & 0.27 & -1367 & 909 & 791 & 575 & 780 & 0.37 \\
\hline Bicycle & 1482 & 1.03 & 0.167 & -1461 & 884 & 791 & 590 & 782 & 0.33 \\
\hline \multicolumn{10}{|l|}{ Crash Type } \\
\hline angle & 4376 & 1.599 & 0.11 & -4346 & 1986 & 791 & 881 & 778 & 0.56 \\
\hline turn & 2478 & 0.76 & 0.07 & -2453 & 1037 & 791 & 713 & 781 & 0.31 \\
\hline straight & 2656 & 1.248 & 0.12 & -2628 & 1539 & 791 & 745 & 779 & 0.52 \\
\hline stop & 4081 & 1.163 & 0.078 & -4055 & 1711 & 791 & 839 & 778 & 0.51 \\
\hline \multicolumn{10}{|c|}{ Crash Severity } \\
\hline FA & 1533 & 1.785 & 0.387 & -1517 & 821 & 791 & 663 & 785 & 0.19 \\
\hline $\mathrm{FI}$ & 4541 & 1706 & 0.116 & -4510 & 1812 & 791 & 889 & 778 & 0.51 \\
\hline I & 4526 & 1.715 & 0.117 & -4494 & 1822 & 791 & 885 & 777 & 0.51 \\
\hline PDO & 5128 & 2.143 & 0.136 & -5098 & 2329 & 791 & 862 & 778 & 0.63 \\
\hline
\end{tabular}




\subsubsection{Estimated Coefficients}

The model coefficients are presented in Table 8: negative coefficients are in red type; statistical significance is indicated by shading, see key for the table.

\subsubsection{Exposure}

Looking at exposure factors in Table 8, VMT is significant in all the models. This would be expected, since the relationship of increased crash rates with increased traffic volume are well established. The volume to capacity ratio, or v/c, is not significant for all models, however. Models of bicycle crashes, angle, and turn crashes, and the higher severity injuries (FA combines fatalities and incapacitating injury severity) did not show this factor as significant. $\mathrm{V} / \mathrm{c}$ could be seen as an indication of congestion, and as such indicates that congestion is less important for crashes of those types. It makes sense that congestion, where more stop and go behavior increases the risk of incidents with one vehicle overtaking another vehicle, would contribute to increased straight and stop crashes.

Average free speed was significant for all three different crash mode models, and for turn and PDO crashes. What is interesting is that the coefficient is negative, implying that a higher speed would decrease the crash risk; typically higher speeds are associated with increased crash rates. This could tie in with congestion: if speeds are lower due to increased congestion, that could explain 
why lower speeds were less safe. Alternatively, it could be due to the quality of the data source, which is based on posted rather than actual speed limits.

Schools were significant for a few crash models: vehicle crashes, angle crashes, FI and I. This again had a negative coefficient, meaning the more schools in an area, the less crashes predicted. This could indicate that efforts to improve traffic safety around schools are indeed effective, slowing drivers down and making them more alert to the potential for pedestrians in the area. With this in mind, it's interesting to note that schools are not significant for the pedestrian crash model, which is the type of crash most school traffic safety policies are targeting.

\subsubsection{Connectivity Factors}

No connectivity factors were statistically significant for vehicle or pedestrian crashes. Several factors were significant for bicycle crashes, though: street network layout of warped parallel and grid network compared to loops and lollipops; intersection density, and total street length. This indicates that bicycles may have a greater crash risk with more streets, particularly more grid like. This may be due to the need to cross intersections, which is indicated by the intersection coefficient being the highest coefficient in the bicycle model.

The percentage of four leg intersections was significant for angle crashes. This is logical due to the fact that four leg intersections have more opportunities 
for angle crashes. Major arterial length was significant for stopped vehicle crashes and injury crashes; block size was also significant for stopped vehicle crashes. This makes sense because major arterials are more likely to have traffic signal control, and shorter block lengths would mean more frequent stop lights, thus more opportunity for rear end collisions.

\subsubsection{Transit accessibility, Demographics, and Origin Destination}

Transit accessibility and origin and destination factors were significant in many models. Transit stops were significant in vehicle, crash severity, and crash type models. Transit stops correlate to employment (see Appendix E), but employment was not significant in most models, only the vehicle crash model, where is had a negative coefficient. Pedestrian crashes showed transit ons and offs to be significant; which is logical since transit riders are pedestrians immediately before and after their transit trip: the higher pedestrian volumes would be expected to increase the possibility for pedestrian crashes.

Population was not significant for bicycle crashes nor for FA injury severity crashes, but was for all other crash models. Business density was significant in all the models. Many trips are due to people getting to and from work, as well as frequenting businesses, so finding these factors significant for crash rates indicates another aspect of exposure to the transportation network. 
Table 8 Model Coefficients

\begin{tabular}{|c|c|c|c|c|c|c|c|c|c|c|c|}
\hline & Vehicle & Ped & Bicycle & angle & turn & straight & stop & FA & FI & I & PDO \\
\hline Intercept & -1.909 & -4.974 & -4.369 & -2.810 & -5.417 & -5.828 & -4.558 & -4.100 & -2.846 & -2.677 & -2.475 \\
\hline \multicolumn{12}{|l|}{ Exposure } \\
\hline $\log (\mathrm{VMT})$ & 0.534 & 0.381 & 0.527 & 0.463 & 0.743 & 0.771 & 0.684 & 0.412 & 0.515 & 0.491 & 0.535 \\
\hline average $\mathrm{v} / \mathrm{c}$ & 0.661 & 1.375 & & 0.115 & & 0.678 & 1.653 & & 0.700 & 0.730 & 0.680 \\
\hline AvgFreeSpeed & -0.012 & -0.015 & -0.054 & -0.009 & -0.026 & -0.009 & -0.003 & 0.008 & -0.004 & -0.006 & -0.013 \\
\hline Schools & -0.093 & & & -0.129 & & & & & -0.113 & -0.113 & -0.082 \\
\hline \multicolumn{12}{|l|}{ Connectivity } \\
\hline $\begin{array}{r}\text { Street Mixed } \\
\text { Warped }\end{array}$ & 0.140 & 0.136 & & 0.256 & 0.395 & 0.060 & 0.065 & & 0.084 & 0.107 & 0.163 \\
\hline Parallel & 0.116 & 0.138 & 0.518 & 0.246 & 0.498 & 0.226 & 0.034 & & 0.108 & 0.162 & 0.167 \\
\hline Street Grid & 0.075 & 0.049 & 0.659 & 0.299 & 0.459 & -0.147 & 0.062 & & 0.075 & 0.166 & 0.186 \\
\hline Intersections & 0.005 & 0.005 & 0.719 & 0.005 & & -0.004 & 0.003 & & 0.001 & -0.008 & 0.001 \\
\hline $\begin{array}{r}\text { FourLegPct } \\
\text { on-Freeway }\end{array}$ & 0.299 & & & 0.847 & & 0.041 & & 0.518 & 0.107 & -0.070 & 0.146 \\
\hline $\begin{array}{r}\text { Street } \\
\text { Major }\end{array}$ & $1.7 \mathrm{E}-05$ & & $8.0 \mathrm{E}-05$ & $1.9 \mathrm{E}-05$ & $2.6 \mathrm{E}-05$ & $2.0 \mathrm{E}-05$ & $-1.4 E-5$ & & $1.4 \mathrm{E}-05$ & $1.3 \mathrm{E}-05$ & $3.3 \mathrm{E}-05$ \\
\hline Arterial length & & & & & $-5.8 \mathrm{E}-05$ & & & & & & \\
\hline $\begin{array}{l}\quad \text { Block Size } \\
\text { Transit } \\
\text { Accessibility }\end{array}$ & & & & & & & & & & $4.2 \mathrm{E}-03$ & \\
\hline Transit Stops & 0.020 & -0.002 & 0.012 & 0.016 & 0.016 & 0.011 & 0.023 & 0.014 & 0.015 & 0.015 & 0.015 \\
\hline Ons \& Offs & $5.6 \mathrm{E}-08$ & $2 \mathrm{E}-07$ & & & & & & & & & \\
\hline \multicolumn{12}{|l|}{ Demographic } \\
\hline Population & $4.9 \mathrm{E}-04$ & $1.3 \mathrm{E}-03$ & & 7.3E-04 & $6.9 \mathrm{E}-04$ & 3.7E-04 & $6.6 \mathrm{E}-04$ & $-8.9 E-5$ & 6.7E-04 & 7.3E-04 & $4.8 \mathrm{E}-04$ \\
\hline \multicolumn{12}{|l|}{ Origin-Dest } \\
\hline Employment & $-1.77 \mathrm{E}-04$ & & & & & & & & & & \\
\hline Business & 0.033 & 0.029 & 0.018 & 0.032 & 0.016 & 0.035 & 0.023 & 0.012 & 0.026 & 0.026 & 0.030 \\
\hline Significance & 0.001 & & 0.01 & & 0.05 & & 0.1 & & & & \\
\hline
\end{tabular}




\subsubsection{Elasticity}

Elasticity gives a measure of relative effect of a factor on the outcome. Elasticity was calculated as follows (17):

$$
E_{x j}=\beta_{j} x_{j}
$$

where $\quad E_{\mathrm{xj}}$ is the elasticity for attribute $j$,

$\beta_{\mathrm{j}}$ is the model coefficient and

$\mathrm{x}_{\mathrm{j}}$ is the mean value for attribute $j$.

\subsubsection{By Vehicle, Pedestrian, and Bicycle Models}

Looking at elasticity for vehicle crashes in Figure 30, VMT had the largest affect. For every additional one percent VMT, there would be an additional 1.7\% increase in vehicle crashes. Street network, non-freeway street length, population and business density had the next strongest affects on crash rate, 0.3 elasticity for mixed and warped parallel compared to loops and lollipops; a lower elasticity of 0.2 for grid layout. Population and business density elasticity were both under 0.3 .

The highest elasticity for pedestrian crashes was 1.3 for VMT, and 0.74 for population. Bicycle crashes have high elasticity for VMT, warped parallel and grid street network, intersections, and non-freeway street length. Average free speed had a high negative elasticity of -0.44 ., indicating that the model predicts less crashes with increased speed. This could be explained if the lower free 
speed is due to heavier congestion and thus higher likelihood of bicycle vehicle interaction, or due to bicyclists avoiding high speed roadways if a lower speed alternate route is available.

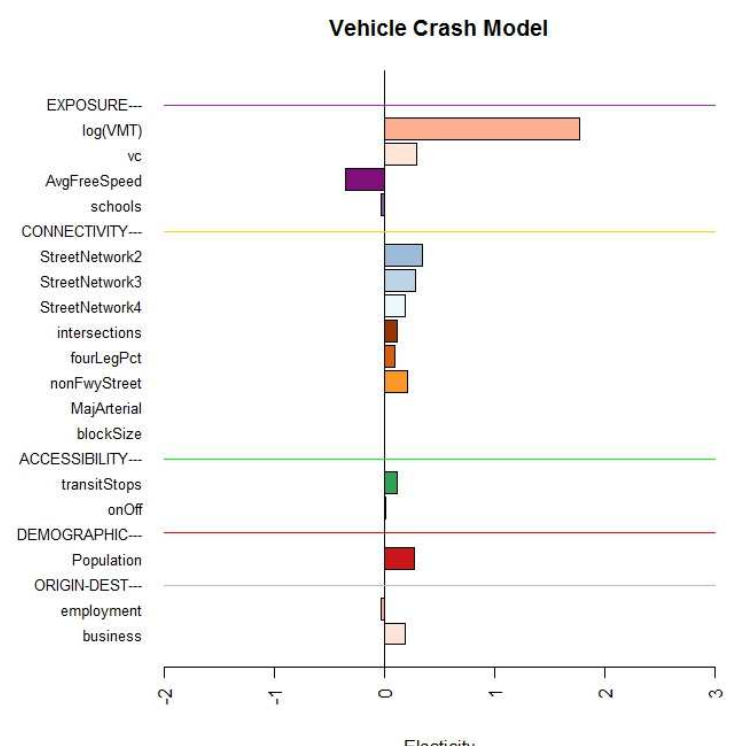

Figure 30a

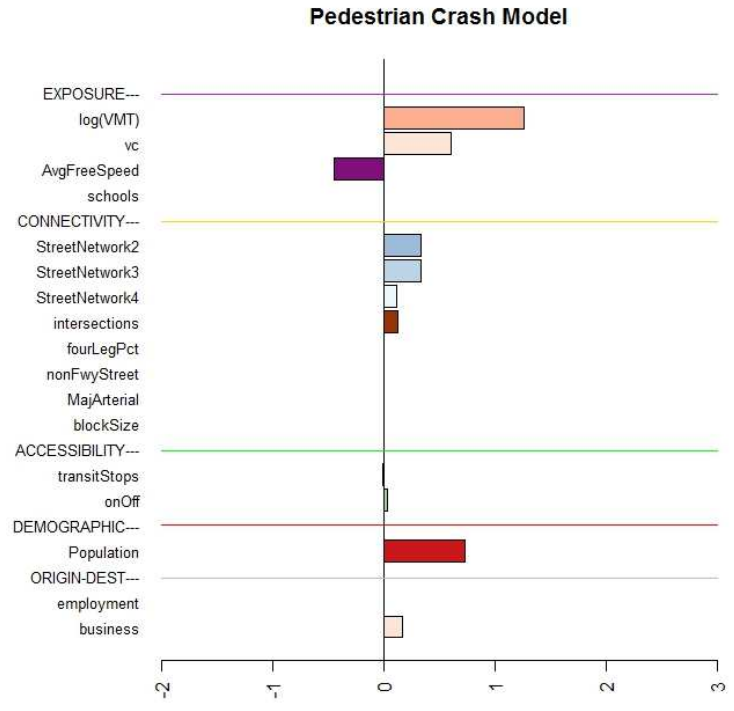

Figure 30b

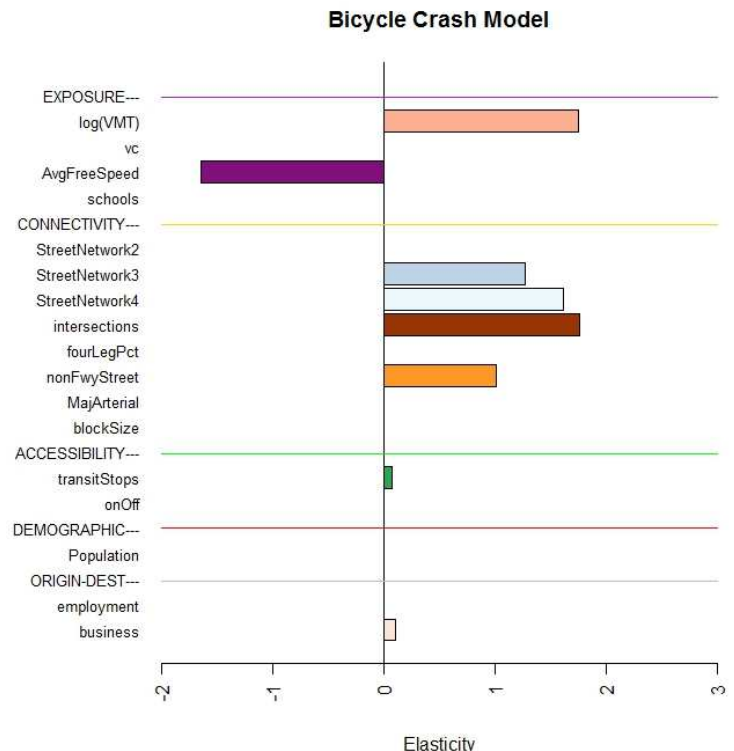

Figure $30 \mathrm{c}$

Figure 30 Elasticity for Vehicle, Pedestrian, and Bicycle Crashes

Page 60 


\subsubsection{Vehicle Crash Types}

Elasticity for crash type models are shown in Figure 31. For all the major crash types, VMT had the highest elasticity. Angle and turn crashes showed positive elasticity for the street layout factors. These crashes are more likely to occur with more intersections; particularly angle crashes at four leg intersections, so this is not surprising. Straight and stopped vehicle crashes had less elasticity for street layout and connectivity factors.

All four crash type models showed a positive elasticity for population and businesses. They also all showed a negative elasticity with average free speed, particularly turn crashes. Lower speeds may encourage drivers pull out of driveways or cross streets when they think they have adequate gap to pullout, but they really don't; whereas higher speed roadways may intimidate drivers to wait for safer gaps. The business count may indicate something about driveways: more businesses typically require more access points, unless design specifically limits access. So the negative elasticity for average free speed and positive elasticity for business density may be related. 


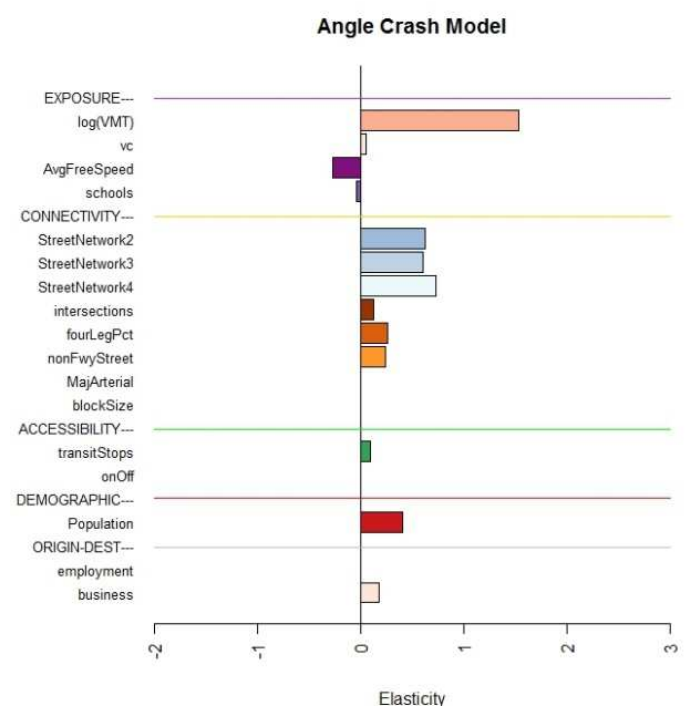

Figure 31a

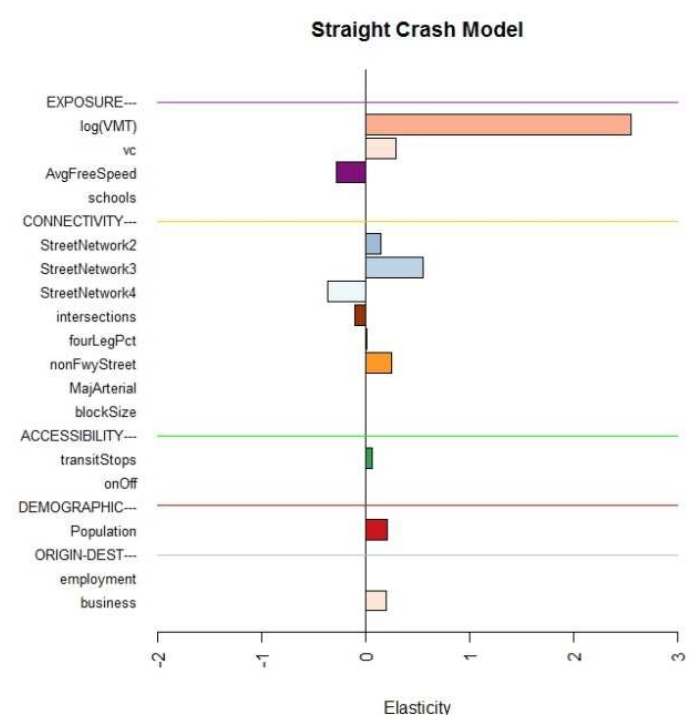

Figure 31c

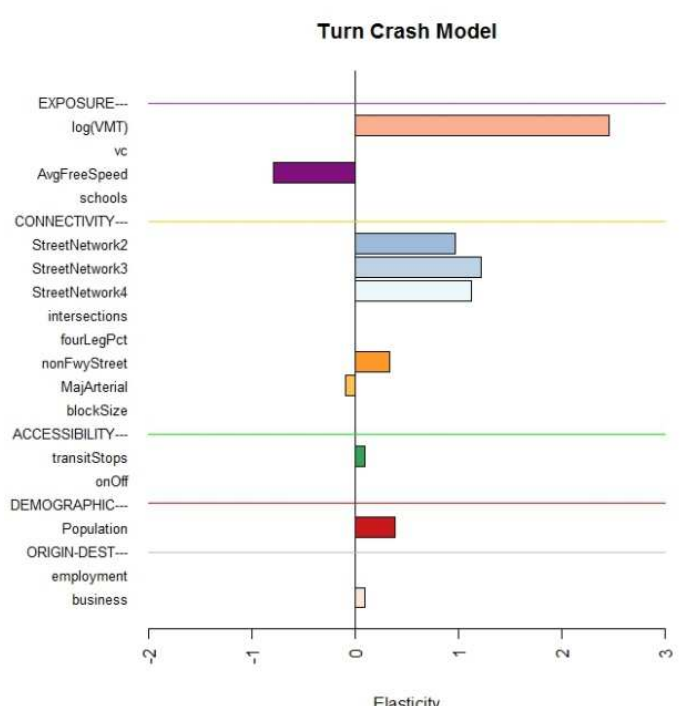

Figure 31b

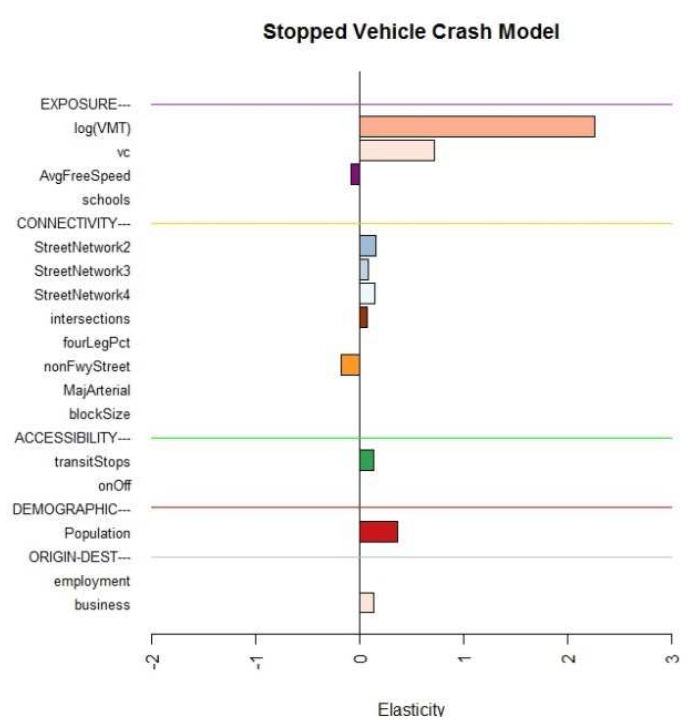

Figure 31d

Figure 31 Elasticity for Vehicle Crash Types

Page 62 


\subsubsection{Crash Severity}

Elasticity for models by crash severity is shown in Figure 32. Again, VMT had the highest elasticity for all crash severities. V/c showed some positive elasticity in the less severe injury categories, but was not in the model for FA, since the factor did not improve that model. FA crashes had few significant factors: VMT, average free speed, percent four leg intersections, transit stops, and business density. This set of significant factors for FA crashes, including the only positive elasticity for average free speed, brings to mind a busy roadway where many things are going on: lots of traffic, moving fast, with transit vehicles and riders in the mix; four legged signalized intersections where drivers may be going through beyond their signal phase; and high business density meaning more access points where vehicles are entering and leaving the transportation network. There are many things going on that could take a driver's attention away from the task of safe driving in this situation, with high speed contributing to less reaction time.

Street network factors had positive elasticity for all three less severe crash models, as do population and businesses. Average free speed was again a negative elasticity. 


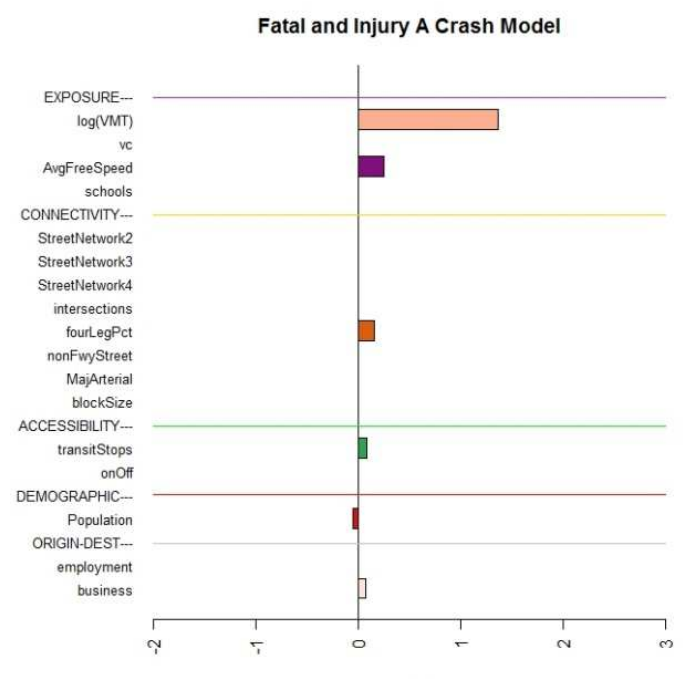

Figure 32a

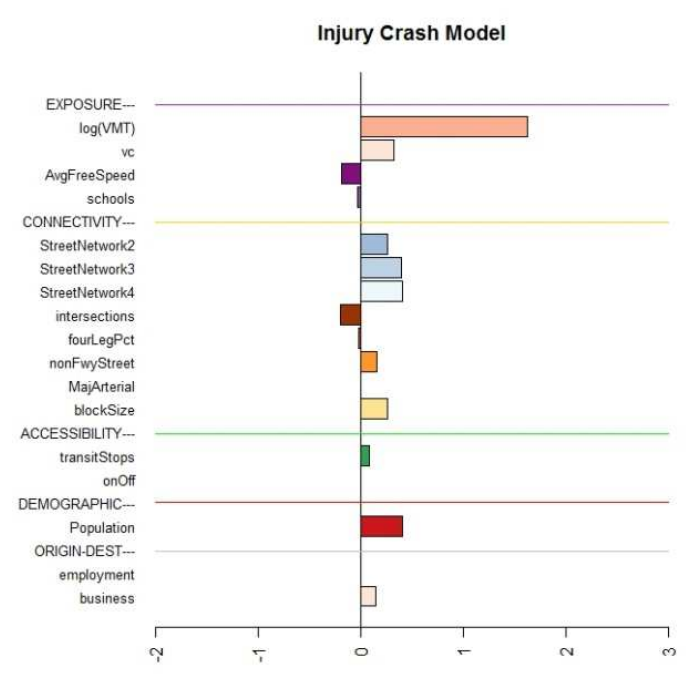

Figure 32c

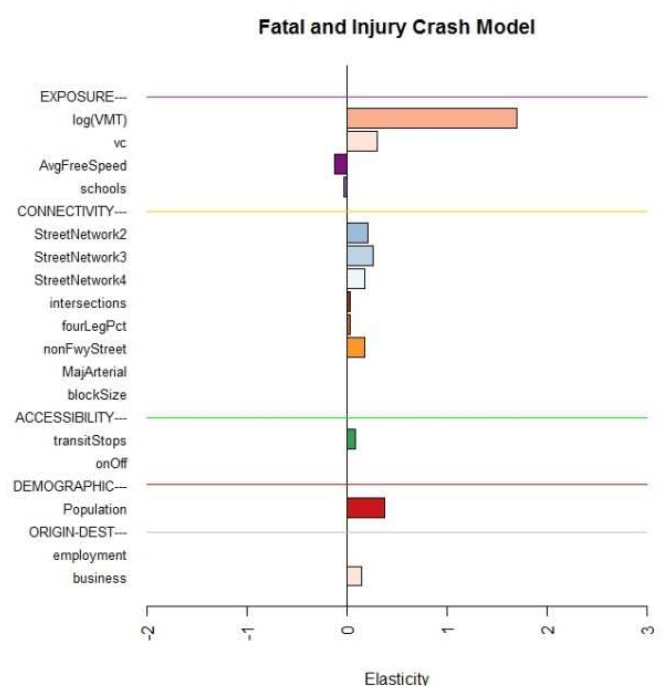

Figure 32b

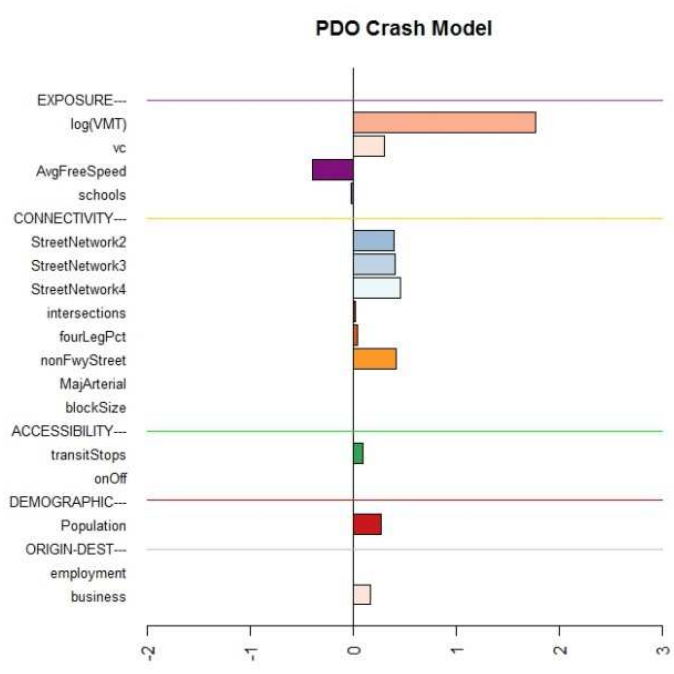

Figure 32d

Figure 32 Elasticity for Crash Severity 


\subsubsection{Elasticity by Factors}

Looking at elasticity by factors in Figure 33, the elasticity for VMT was high for all models, particularly for turn, straight, and angle crashes. This indicated that increased traffic in an area greatly increased the probability of crashes. Straight crashes had the highest elasticity. This may be due to increased VMT in urban areas usually being due to increased congestion, causing vehicles to pack themselves into tighter space, with less distance between vehicles. Decreased distance between vehicles gives a driver less time to respond to a sudden deceleration of a vehicle in front of them, which could lead to a straight or stopped vehicle crash.

Volume to capacity $(\mathrm{v} / \mathrm{c})$ had highest elasticity in the pedestrian and stopped vehicle crash models. Increased v/c could mean more congestion, leaving less space for pedestrians and vehicles to stay separated. The less severe and non-injury crash models also showed sensitivity to v/c.

Average free speed had negative elasticity for every model except FA. Bicycle crashes had the strongest negative elasticity, which seems counter intuitive. This may be due to bicyclists tending to prefer lower speed routes to riding alongside higher speed traffic. In lower speed conditions, bicycle riders may take advantage of their size and squeeze between or past vehicles stopped at traffic signals or stop sings, putting them at greater risk of collision. The most 
severe injuries, FA crashes, had the only positive elasticity for average free speed, strongly suggesting a link between speed and severe injury.

Elasticity for street network compared to loops and lollipops can be seen in Figure 34. These factors had especially high elasticity for bicycle and turn crashes, as was discussed going through elasticity by model. Bicycle crashes also had high elasticity for intersection density, percent four leg intersections, and total street length (see Figure 35).

Population elasticity was highest for the pedestrian crash model. This makes sense since the more people there are, the more pedestrians . Population elasticity was higher for the less severe crash types.

Similarly business had positive elasticity as well, with less of an effect on FA crashes than other severity levels. The positive elasticity all models may be due to the fact the a higher count of businesses probably means more smaller businesses, which would each have a separate location, and thus more driveways for people to get to and from the businesses. Driveways have been associated with increased crash risk. 

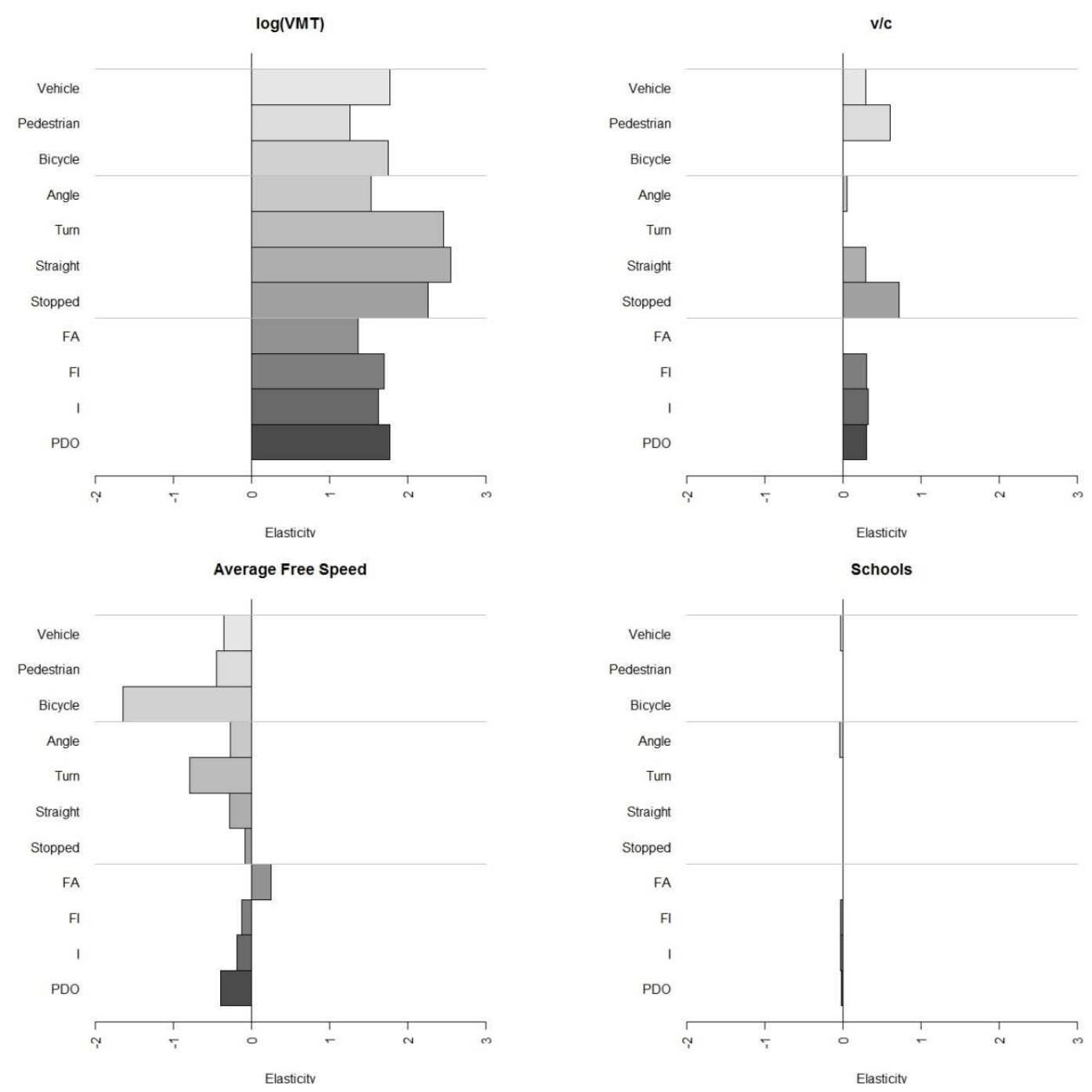

Figure 33 Elasticity for Exposure Factors

Page 67 

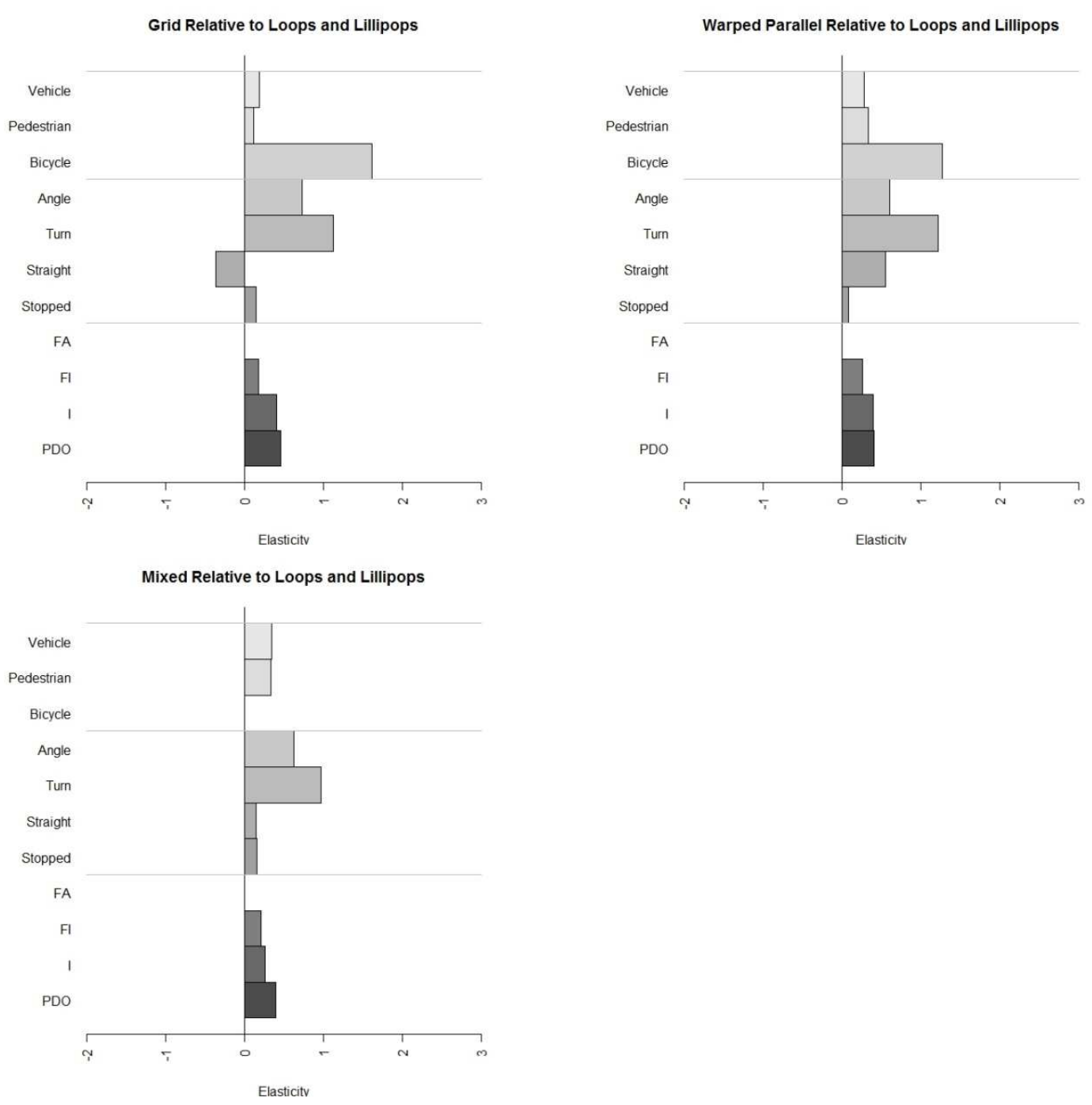

Figure 34 Elasticity for Street Network Factors

Page 68 

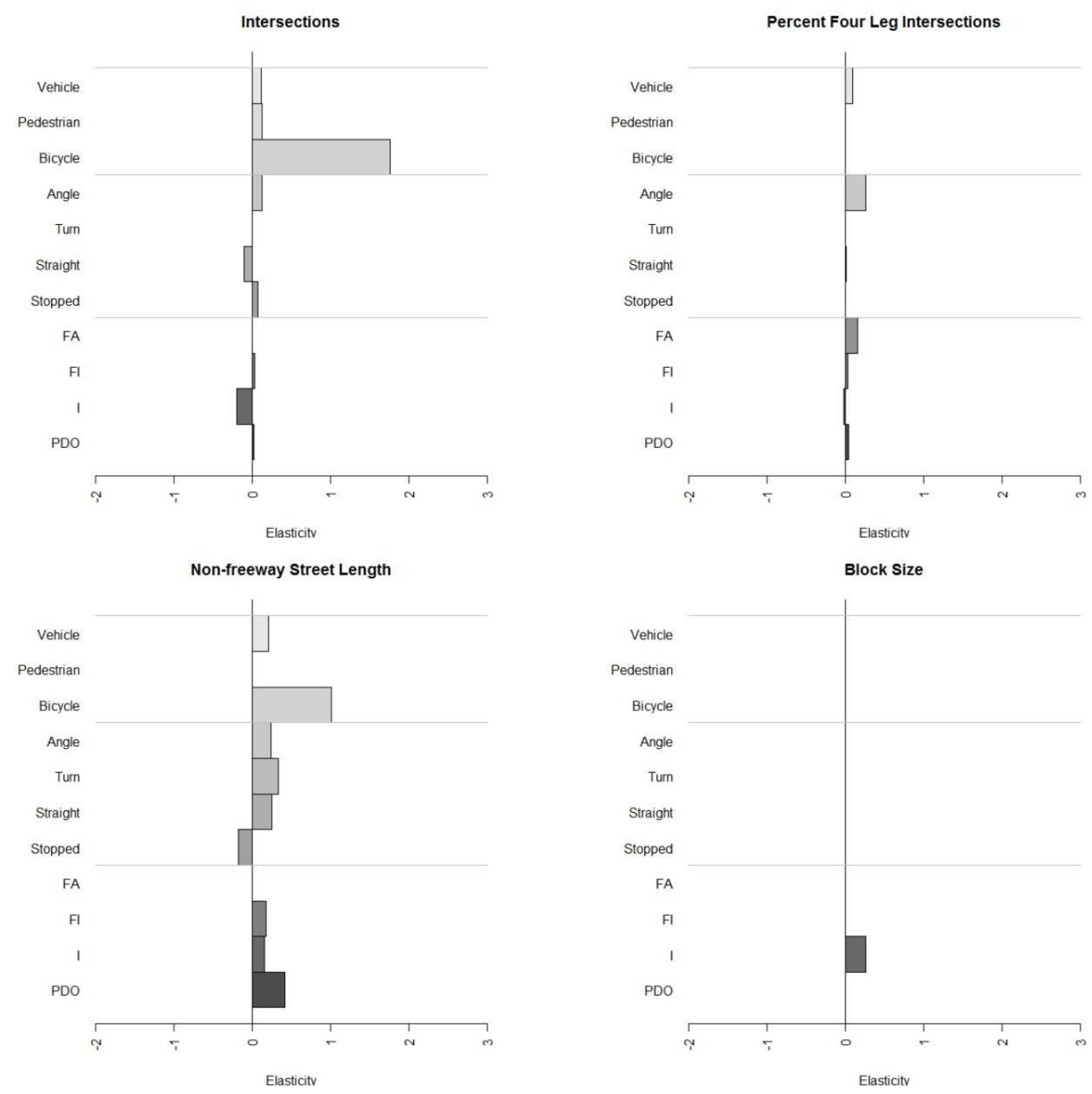

Figure 35 Elasticity for Connectivity Factors

Page 69 


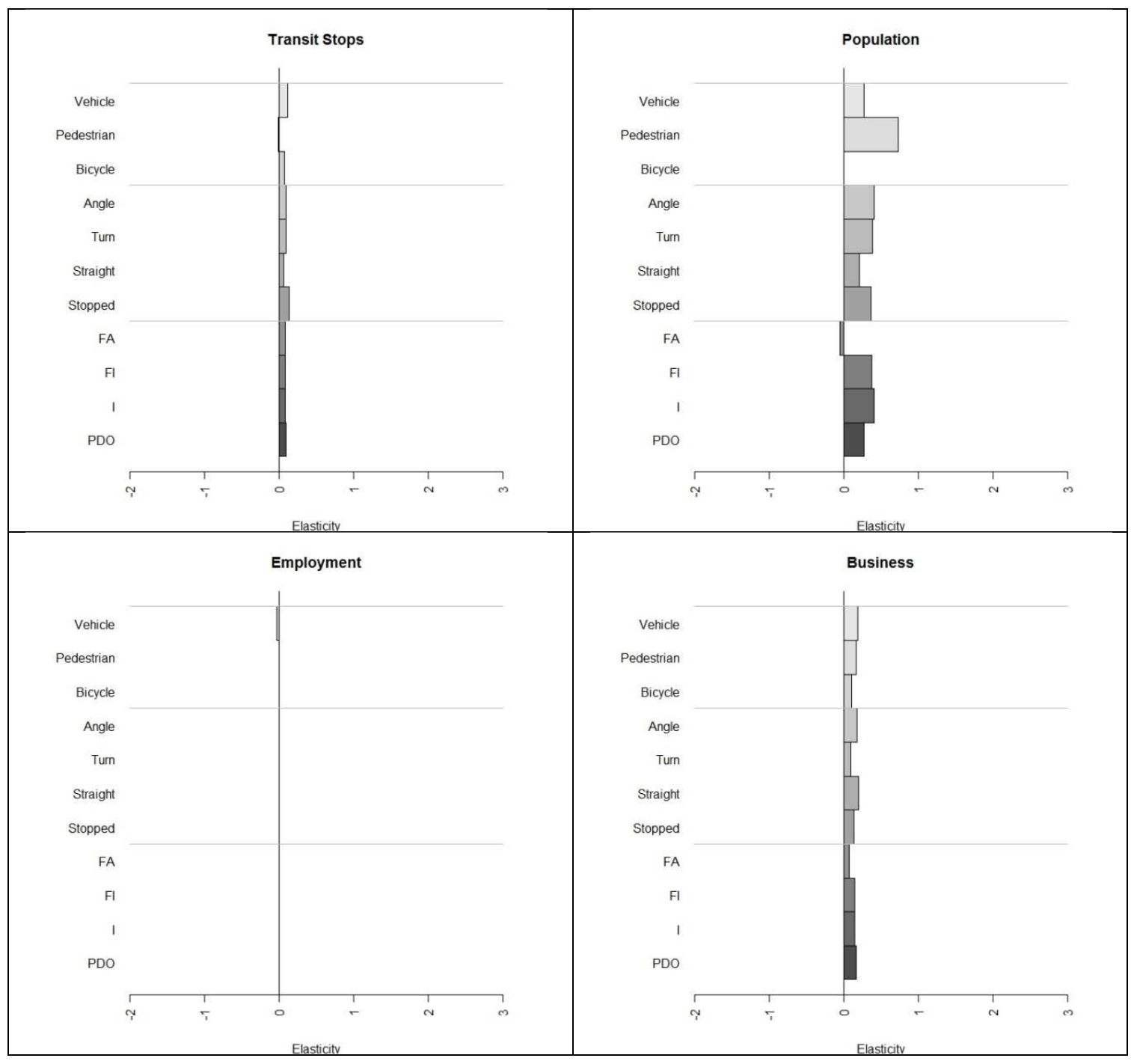

Figure 36 Elasticity for Transit Accessibility, Demographic, Origin and Destination Factors

Page 70 


\subsection{CONCLUSIONS}

Street layout and design, once established, are then not easily changed. Urban form affects community development, livability, sustainability, and traffic safety. There has been an assumed relationship between urban form and traffic safety that favors designs with less through streets to improve safety. An empirical study to test this assumed relationship was carried out for crash data for Portland, Oregon, considering factors for exposure, connectivity, transit accessibility, demographics, and origin and destination measures.

This study looked at traffic safety and urban form for the city of Portland, Oregon using a uniform spatial grid to provide an impartial way to assign crashes to the analysis spatial units. Data were assigned to each spatial grid cell by summing point data, line data, or apportioning data from a different underlying polygon spatial unit into the grid cell spatial unit.

In qualitative analysis of chloropleths showing the spatial distribution of the crashes in the grid cell aggregation, major arterials and high volume roadways clearly stood out as having more crashes. Comparing the 20,705 nonfreeway vehicle crashes to 503 pedestrian and 523 bicycle crashes indicated much higher and more severe injury rates for pedestrian and bicycle crashes than in vehicle crashes: the pedestrian fatality rate is more than ten times that 
for vehicles, and the bicycle fatality rate is nearly four times that for vehicle crashes. The pedestrian or bicyclist involved in a crash with a vehicle has little protection against the order of magnitude greater mass and momentum of the vehicle.

Qualitative analysis of the exposure factors showed higher volumes along major arterials and in the downtown area, as would be expected. Connectivity factors for street network using the four Rifaat and Tay designations (4) were assigned to each spatial grid cell, and visually correlated to a calculable factor of percentage four leg intersections. Transit stop locations gave a view of transit accessibility. Demographic factors of population, households, and dwelling units were highly correlated; only population was included in modeling. Distribution of employment and businesses throughout the study area could also be seen in the chloropleths for those factors. Employment was highly concentrated in the downtown area and other specific clusters. Business density was also concentrated downtown and on the near east side, but was distributed throughout the city more so than the employment, indicating smaller businesses employing fewer workers.

Negative binomial regression models were built separately for groups of vehicle, pedestrian, and bicycle crashes. Models were also built for the top four crash types, as well as by crash severity. The selected models showed that 
exposure factors were significant in all models studied: higher traffic volumes and congestion increased crash likelihood. Exposure factors also had the highest elasticity, indicating that crash rates have strong sensitivity to these volume factors. Average free speed had a surprising negative elasticity, particularly for bicycle crashes.

Elasticity for connectivity factors showed bicycle, angle, and turn crashes to be particularly sensitive to a more grid like street network, compared to loops and lollipops. Street network was a factor in less severe crashes, but was not seen as a factor in incapacitating or fatal crashes. FA crashes were the only model to have a positive elasticity for average free speed, indicating that speed can be a strong factor in severe injury crash rates.

Urban form factors of street connectivity and intersection density were not significant at $95 \%$ confidence for vehicle and pedestrian crash models, nor for different crash severity levels. This indicates that street layout in terms of grid versus loops and lollipops does not have a statistically significant effect on vehicle crash safety, so connectivity does not have to be sacrificed in the name of safety for vehicles or pedestrians. Other factors, such as VMT, v/c, population, and business density, are far more influential.

Several origin and destination factors were significant in the models. Business density was significant for all crash models. Business density could 
indicate the number of access points into the transportation network. Driveways increase crash rates, so the significance of the business factor may be due to increased driveways and access points. If so, limited access design could help control and mitigate crash risk along corridors.

Population was also significant in many models. Although logical, this dependence of crashes on business and population densities raises concerns about vibrant, economically vital areas where businesses, pedestrians, bicyclists, and transit thrive alongside vehicle traffic. Thriving neighborhoods are at the heart of successful development. These results should be seen to highlight the important effect planning and directing development for where businesses, employment, and housing will grow potentially has on safety, and stress that design and planning include plans for traffic safety. Portland Metro is working on planning for major corridors which handle large traffic volumes to serve multiple transportation modes as "complete" streets that are safe for all modes, and attract people to spend time and enjoy their streets.

This study makes a contribution to the study of traffic safety and urban form in having found that connectivity factors for street layout are not statistically significant for vehicle and pedestrian crash rates. This substantiates that grid street layout designs, which provide high connectivity and thus alternative routes to allow large traffic volumes to pass through an area, are not 
sacrificing traffic safety compared to limited access loops and lollipops, despite long held assumptions that limited access designs are better in terms of traffic safety.

The methodology of aggregating crashes and factors into a uniform spatial grid for traffic safety analysis, an approach suggested by Kim (13), provides a way to include all crashes for analysis regardless of whether they were located along arterials that would border TAZ or block group spatial units. Previous researchers have looked at the relationship of traffic safety and urban form using TAZ or block group spatial units. These spatial unit choices were problematic regarding how to handle crashes on the peripheral roadways. The uniform spatial grid methodology gives equal weight to all crashes for an unbiased look at the overall traffic safety, so that safety for neighborhoods streets and arterials can both be considered.

\subsection{Further Work}

The model could be applied to data for years later than the study, when they are available, to see how well the models predicted Portland City crashes. A larger study area could be considered if geo-coded crash data are available, and using connectivity factors such as percent four leg intersections, total intersection density, and total street length, rather than needing to manually assign the Rifaat and Tay street network scale.

Page 75 
Data on volume for more streets would be expected to improve the model. Arterial congestion data would be particularly interesting to study along with speed data to see if there are interactions or relationships there that affect crash rates, particularly for bicycle crashes. This study had data for vehicle roadways; further studies with bicycle and pedestrian facility and volume data could be illuminating for pedestrian and bicycle crash safety.

Future work could consider looking at spatial proximity effects on the analysis. Spatial correlation likely exists, but we did not develop models to account for this. Even though freeway roadways and crashes were eliminated from the study, a dummy variable indicating whether a spatial grid cell included freeway could be included to look for whether areas adjacent to freeways have the same or higher crash rates than non-freeway adjacent areas.

Specific locations could be looked at in more detail, and a mean time to failure (i.e. crash) analysis approach. 


\subsection{REFERENCES}

1. Southworth M, Ben-Joseph E. Streets and the shaping of towns and cities. New York: McGraw hill; 1997.

2. Kim K, Brunner I, Yamashita E. Influence of Land Use, Population, Employment, and Economic Activity on Accidents. In Transportation Research Record: Journal of the Transportation Research Board 2006;1953:56-64.

3. Dumbaugh E, Rae R. Safe Urban Form: Revisiting the Relationship Between Community Design and Traffic Safety. Journal of the American Planning Association 2009;75(3):309.

4. Rifaat SM, Tay R. Effects of Street Patterns on Injury Risks in Two-Vehicle Crashes. In Transportation Research Record: Journal of the Transportation Research Board 2009;2102:61-67.

5. Rifaat S, Tay R. Effect of Street Pattern on Road Safety: Are Policy Recommendations Sensitive to Different Aggregations of Crashes by Severity? Presented at the 89th Annual Meeting of the Transportation Research Board; 2010

6. Marks H. Subdividing for Traffic Safety. Traffic Quarterly 1957;11(2):308-325.

7. Kim K, Levine N. Using GIS to improve highway safety. Computers, Environment and Urban Systems 1996;20(4-5):289-302.

8. Kim K, Levine N, Nitz L. Development of a prototype traffic safety geographic information system. In Transportation Research Record 1995;(1477):41-47.

9. Ossenbruggen $P$, Pendharkar J, Ivan J. Roadway safety in rural and small urbanized areas. Accident Analysis and Prevention 2001;33(4):485-498.

10. Hadayeghi A. Macrolevel Accident Prediction Models for Evaluating Safety of Urban Transportation Systems. In Transportation research record 2003;03611981(1840):87. 
11. Ladrón de Guevara F, Washington S, Oh J. Forecasting Crashes at the Planning Level: Simultaneous Negative Binomial Crash Model Applied in Tucson, Arizona. In Transportation Research Record: Journal of the Transportation Research Board 2004 Jan;1897(-1):191-199.

12. Al-Masaeid HR, Suleiman G. Relationships between urban planning variables and traffic crashes in Damascus. Road and Transport Research 2004;13(4):63-73.

13. Kim K, Pant P, Yamashita E. Accidents and Accessibility: Measuring the Influences of Demographic and Land Use Variables in Honolulu, Hawaii. Presented at the 89th Annual Meeting of the Transportation Research Board; 2010

14. Clifton $\mathrm{KJ}$, Burnier CV, Akar G. Severity of injury resulting from pedestrianvehicle crashes: What can we learn from examining the built environment? In Transportation Research Part D: Transport and Environment 2009 Aug;14(6):425-436.

15. Lord D, Mannering F. The Statistical Analysis of Crash-Frequency Data: A Review and Assessment of Methodological Alternatives. Transportation Research, Part A 2010;44(5):291-305.

16. Hauer E, Bamfo J. Two Tools for Finding What Function Links the Dependent Variable to the Explanatory Variables. Proceedings of ICTCT 97 Conference 1997 Nov;

17. Strathman JG, Dueker KJ, Zhang J, Williams T. Analysis of Design Attributes and Crashes on the Oregon Highway System. Oregon Department of Transportation, Research Unit; 2001. 


\section{APPENDIX A: R code for the CURE plots}

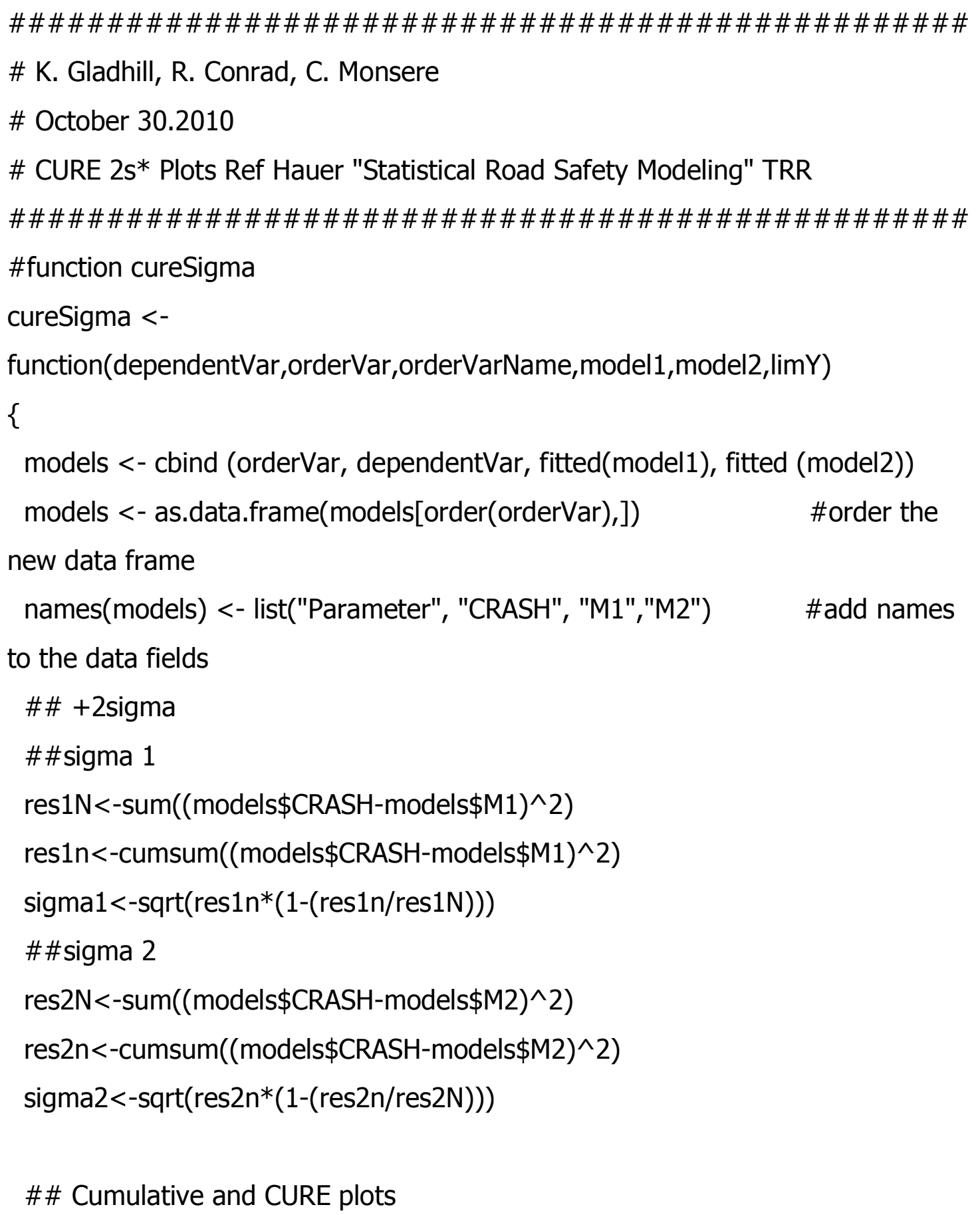


\# find the max value for the $y$-axis before opening plot window

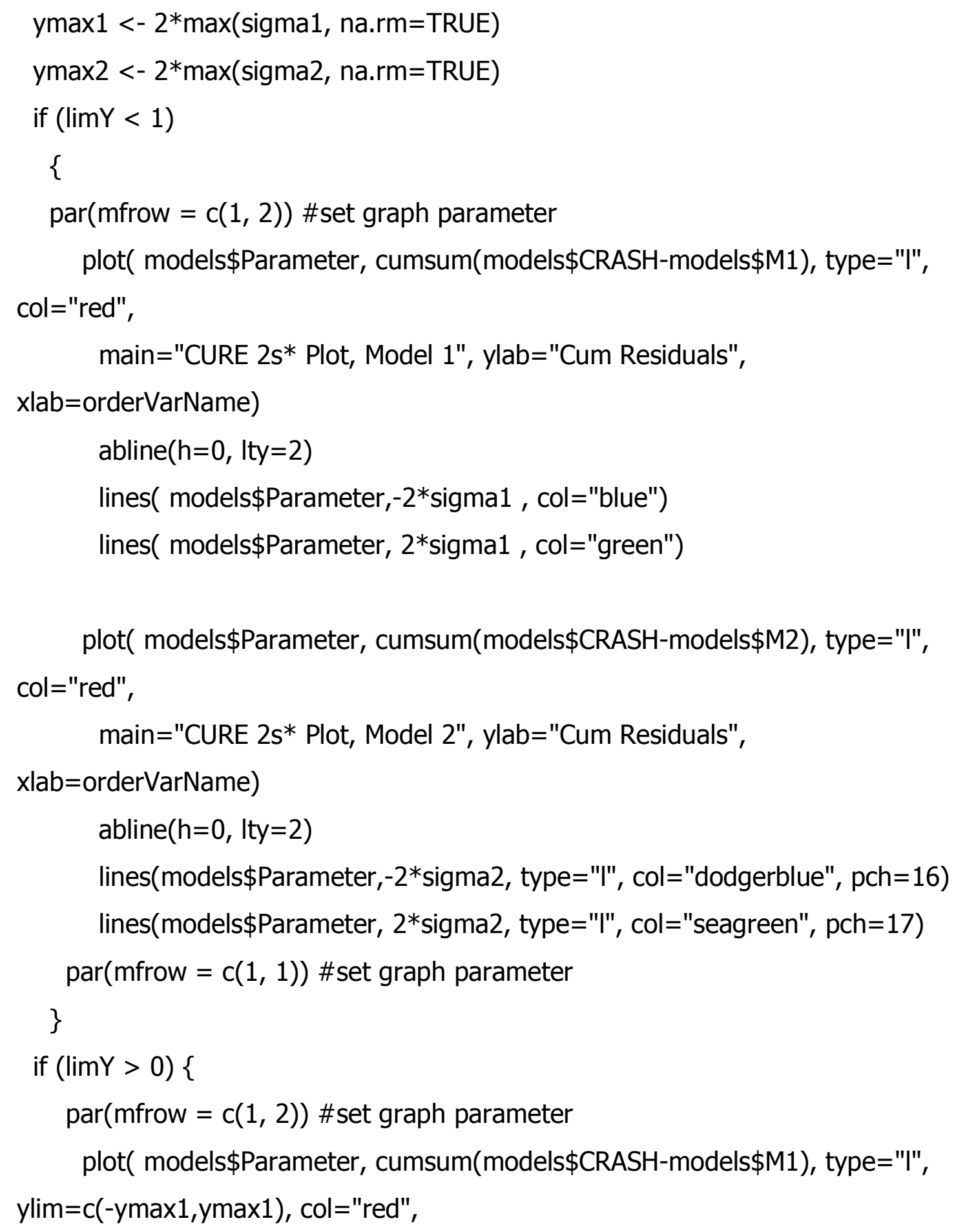




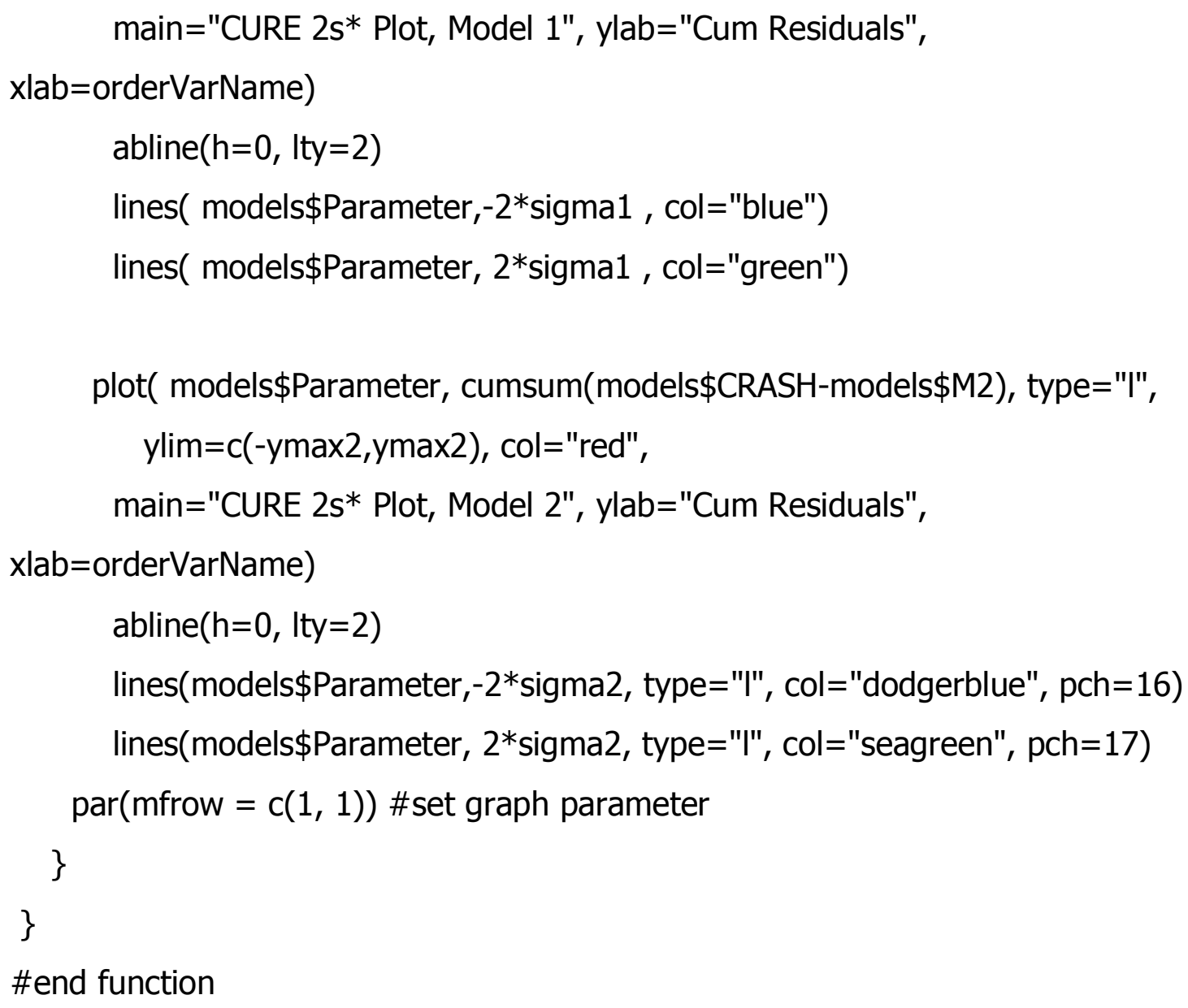




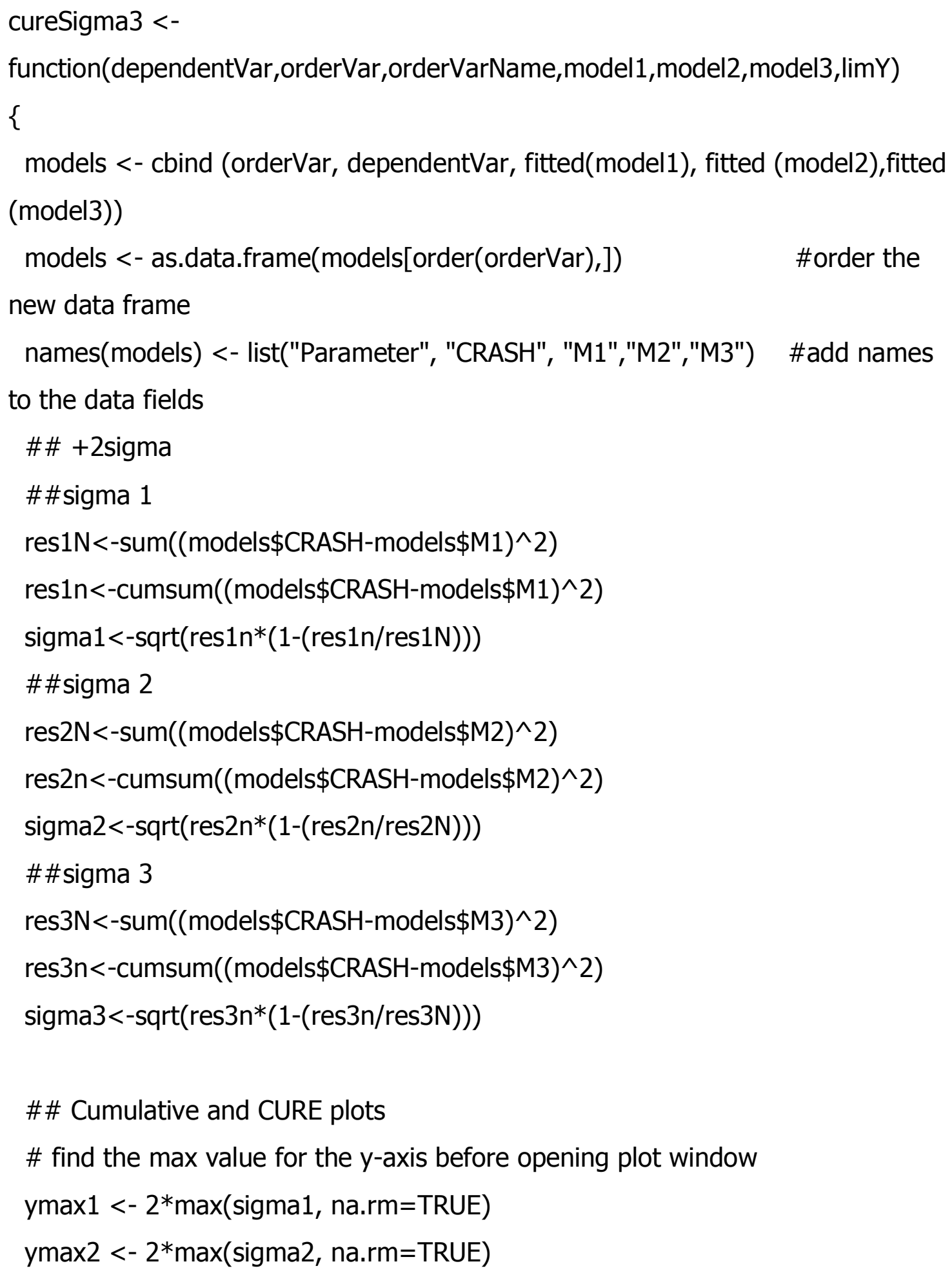




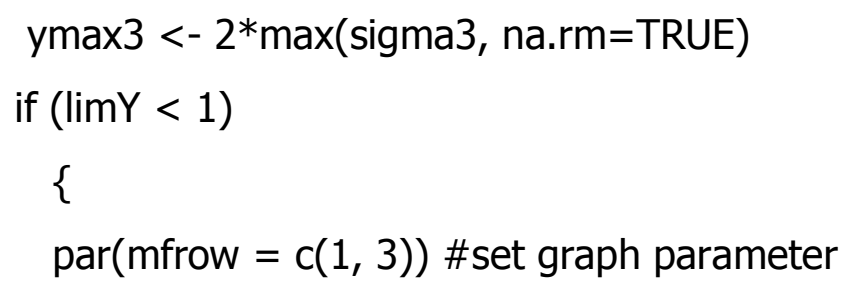

plot( models\$Parameter, cumsum(models\$CRASH-models\$M2), type="l", col="red", main="CURE 2s* Plot, Model 2", ylab="Cum Residuals", xlab=orderVarName)

abline $(\mathrm{h}=0, \mathrm{lty}=2)$

lines(models\$Parameter,-2*sigma2, type="l", col="dodgerblue", pch=16) lines(models\$Parameter, 2*sigma2, type="l", col="seagreen", pch=17)

plot( models\$Parameter, cumsum(models\$CRASH-models\$M3), type="l", col="red", main="CURE 2s* Plot, Model 3", ylab="Cum Residuals", $\mathrm{xlab}=$ orderVarName) abline $(\mathrm{h}=0$, Ity $=2)$ lines(models\$Parameter,-2*sigma3, type="l", col="dodgerblue", pch=16) 
lines(models $\$$ Parameter, $2 *$ sigma3, type $=" l "$, col="seagreen", pch=17)

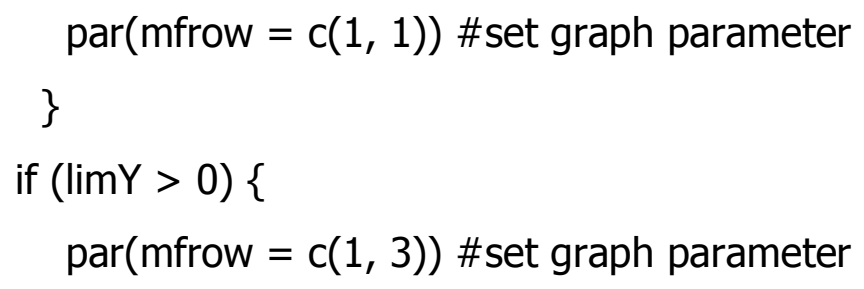

plot( models\$Parameter, cumsum(models\$CRASH-models $\$$ M1), type="l", ylim=c(-ymax1,ymax1), col="red", main="CURE 2s* Plot, Model 1", ylab="Cum Residuals", $\mathrm{xlab}=$ orderVarName) abline $(\mathrm{h}=0, \mathrm{Ity}=2)$ lines( models\$Parameter,-2*sigma1, col="blue") lines( models\$Parameter, 2*sigma1, col="green")

plot( models\$Parameter, cumsum(models\$CRASH-models\$M2), type="l", ylim=c(-ymax2,ymax2), col="red", main="CURE 2s* Plot, Model 2", ylab="Cum Residuals", $\mathrm{xlab}=$ orderVarName) abline $(\mathrm{h}=0, \mathrm{Ity}=2)$ lines(models\$Parameter,-2*sigma2, type="l", col="dodgerblue", pch=16) lines(models $\$$ Parameter, $2 *$ sigma2, type $=" l "$, col="seagreen", $p c h=17$ )

plot( models\$Parameter, cumsum(models\$CRASH-models $\$ M 3)$, type $=" I "$, ylim=c(-ymax2,ymax2), col="red", main="CURE 2s* Plot, Model 3", ylab="Cum Residuals", $\mathrm{xlab}=$ orderVarName) 


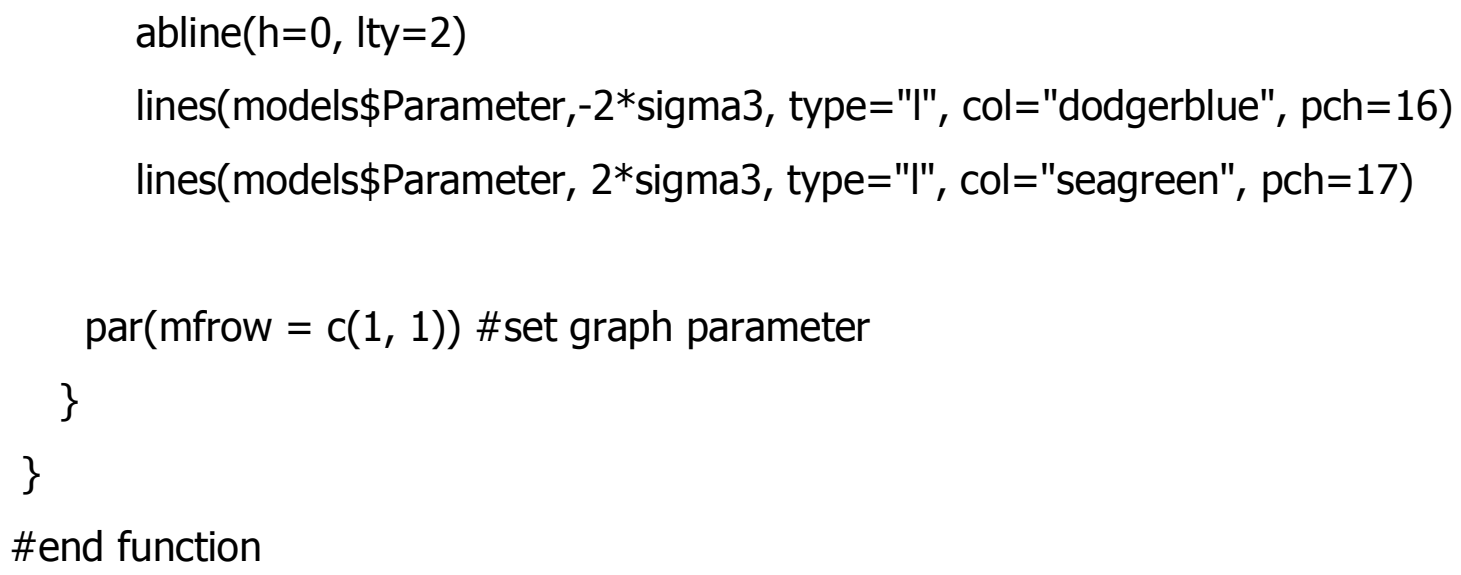




\section{APPENDIX B: Join point data to spatial area in ArcGIS 9.3.1}

Join point data to spatial area

a. Choose layer with point data, start join from there:

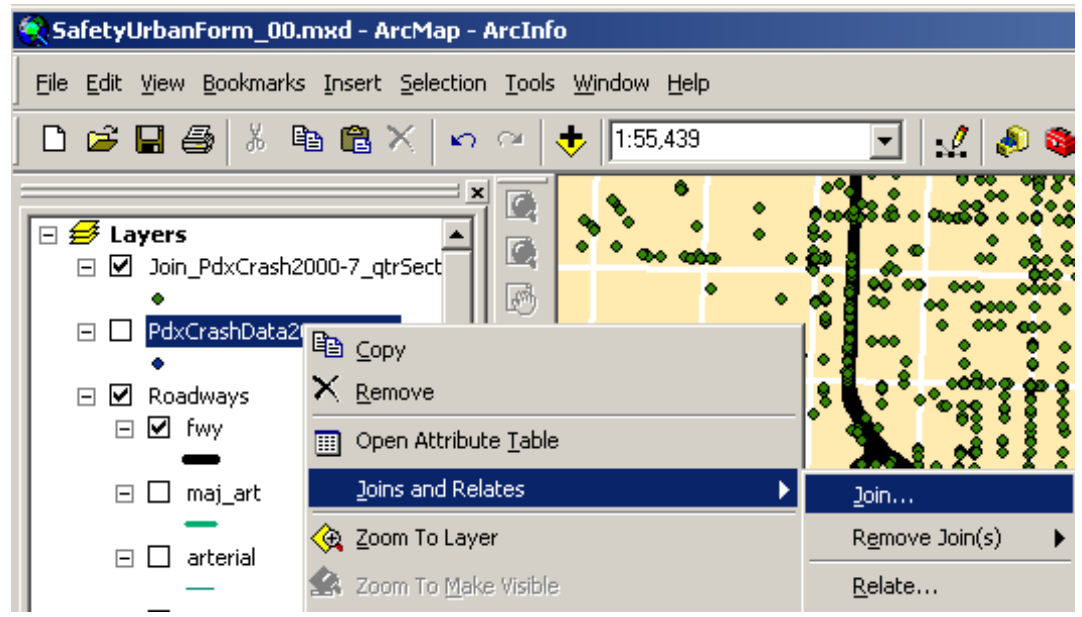

Join Data
Join lets you append additional data to this layer's attribute table so you can,
for example, symbolize the layer's features using this data.
What do you want to join to this layer?
Join data from another layer based on spatial location
1. Choose the layer to join to this layer, or load spatial data from disk:
2. You are joining: Polygons to Points
Select a join feature class above. You will be given different
options based on geometry types of the source feature class
and the join feature class.
Each point will be given all the attributes of the polygon that:
- it falls inside.
If a point falls inside more than one polygon (for example,
because the layer being joined contains overlapping polygons)
the attributes of the first polygon found will be joined.
About Joining Data
is closest to it.
A distance field is added showing how close the polygon is (in
the units of the target layer). A polygon that the point falls
inside is treated as being closest to the point (i.e. a distance
of 0 ).
Specify gutput shapefile or feature class for this new layer:
the result of the join will be saved into a new layer.

This may take some time. 
b. In joined attribute table, choose column to summarize on. In this case, a count of field "Col_Row" will do, it's the ID for each grid cell.

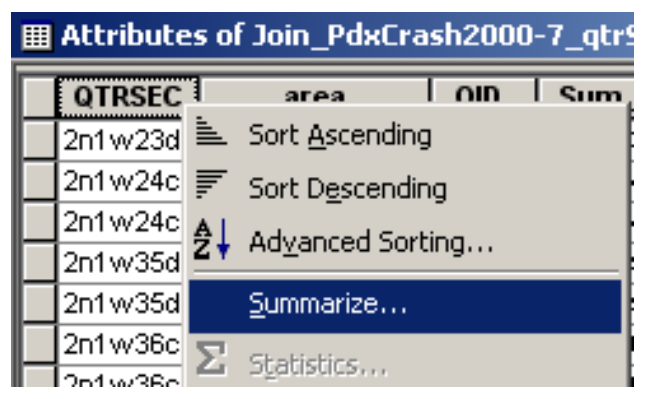

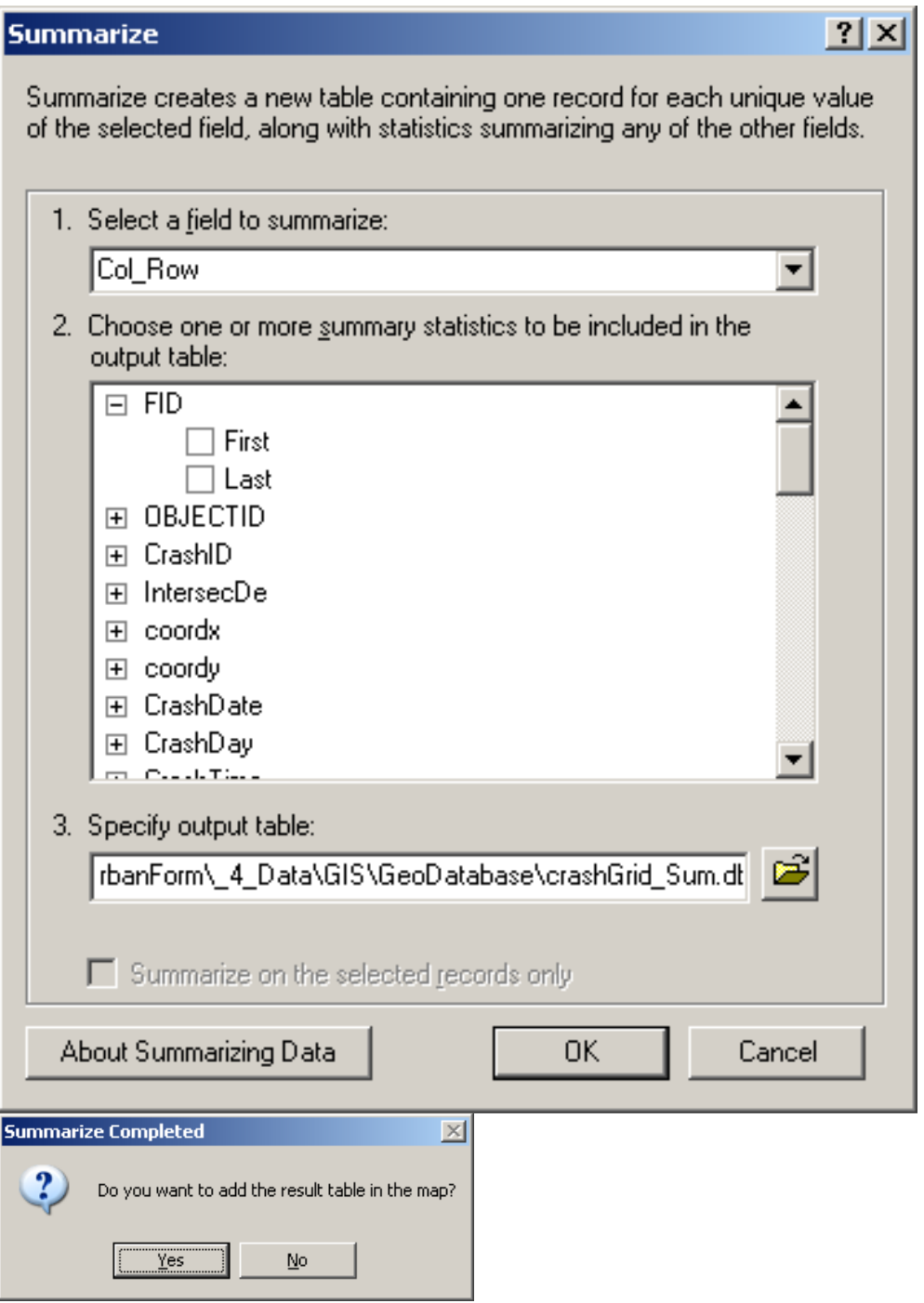

Page 87 
c. Right-click on the table on the "Source" tab to open that table to check data in the summary column:

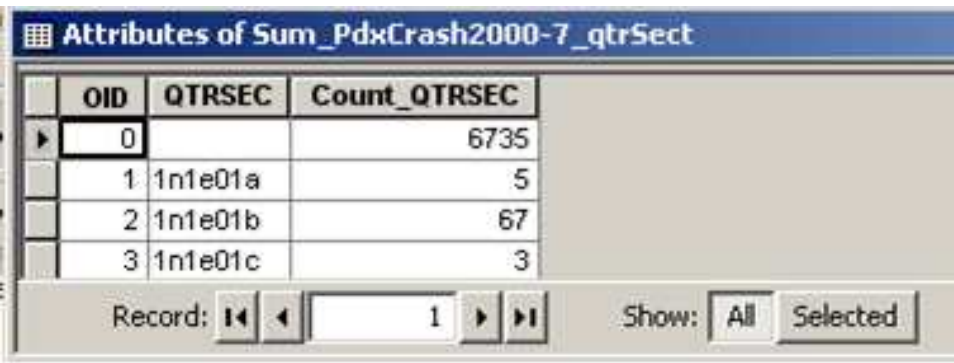

d. Join summary data to the original spatial Layer.

Start with R-click of spatial layer, choose Joins and Relates -> Join 


Join Data
Join lets you append additional data to this layer's attribute table so you can,
for example, symbolize the layer's features using this data.
What do you want to join to this layer?
Join attributes from a table
1. Choose the field in this layer that the join will be based on:
2. Choose the țable to join to this layer, or load the table from disk:
Row 国 crashGrid_Sum
3. Choose the field in the table to base the join on:
Col_Row
Join Options
About Joining Data
All reecords in the target table are shown in the resulting table.
Unmatched records will contain null values for all fields being
appended into the target table from the join table.
C Keep only matching records
If a record in the target table doesn't have a match in the join
table, that record is removed from the resulting target table.

e. Check the attributes table for spatial layer, it now includes columns from the joined table.

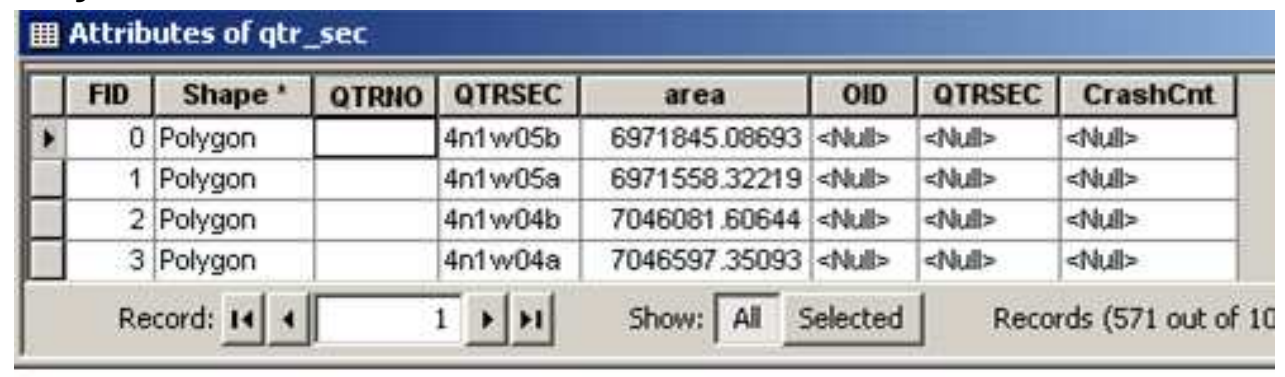




\section{APPENDIX C: Join Line Data to spatial area in ArcGIS 9.3.1}

Determine the length of line data in polygons

1. Create a new output layer is containing road segments intersecting grid cells, clipped at each cell.

Analysis Tools -> Overlay - > Intersect

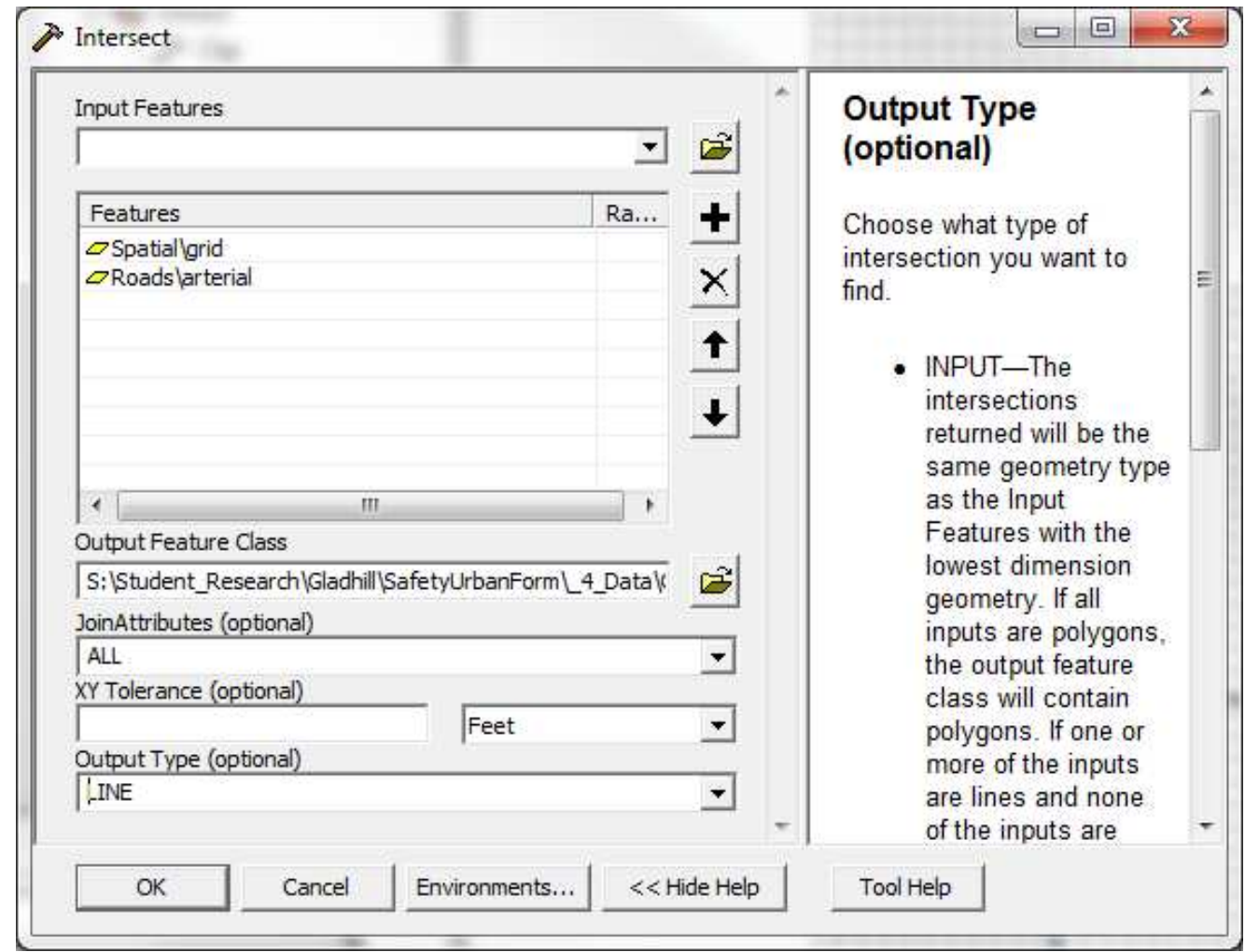


2. Sum the lines that fall within the polygons

a. ArcToolbox, 'Analysis Tools -> Statistics ->Summary Statistics

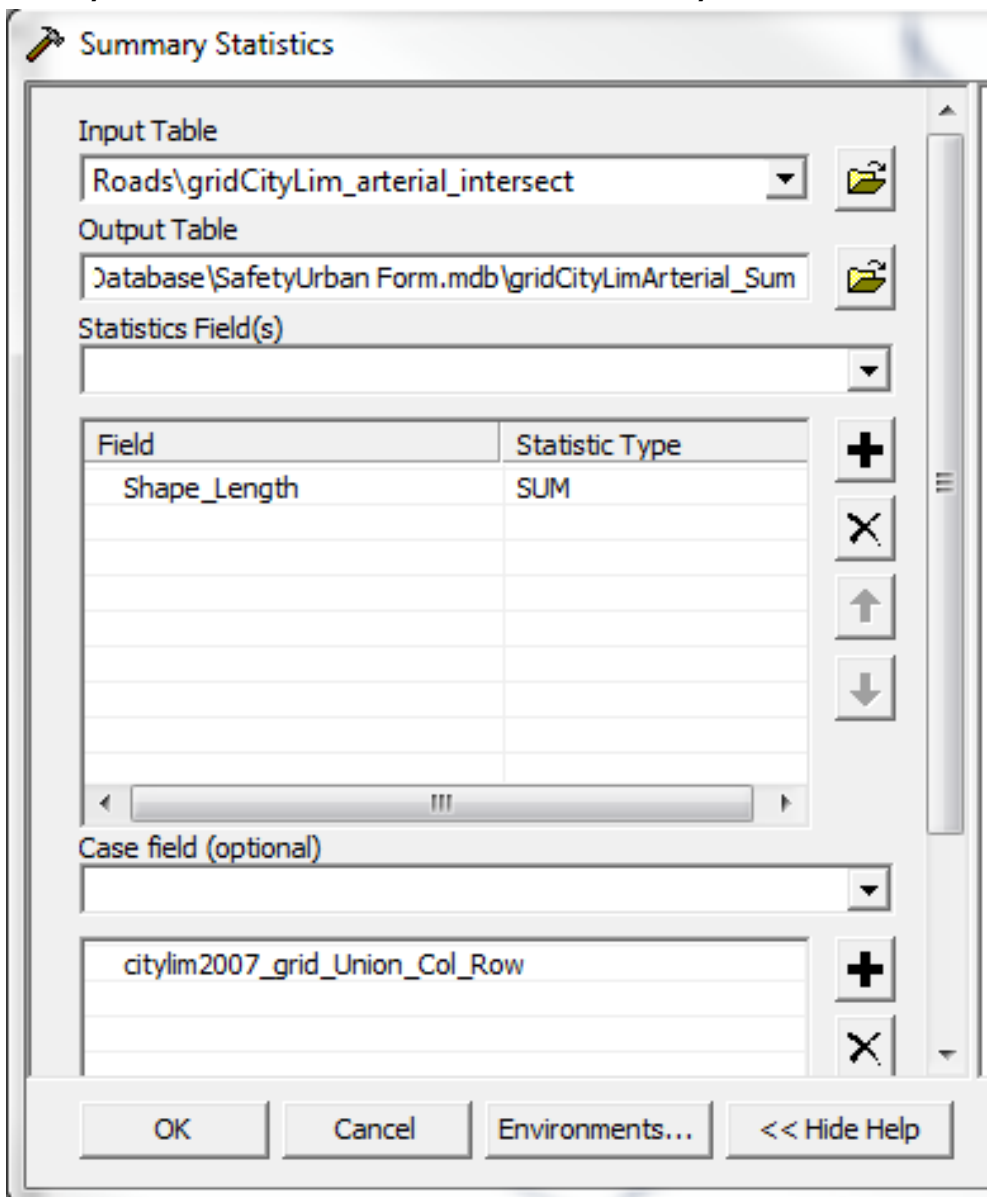

Hit OK, and Close-it will look like nothing happened...that's OK. 
b. In the Table of Contents window, Right-click the polygon layer and select : Join and Relates $->$ Join...

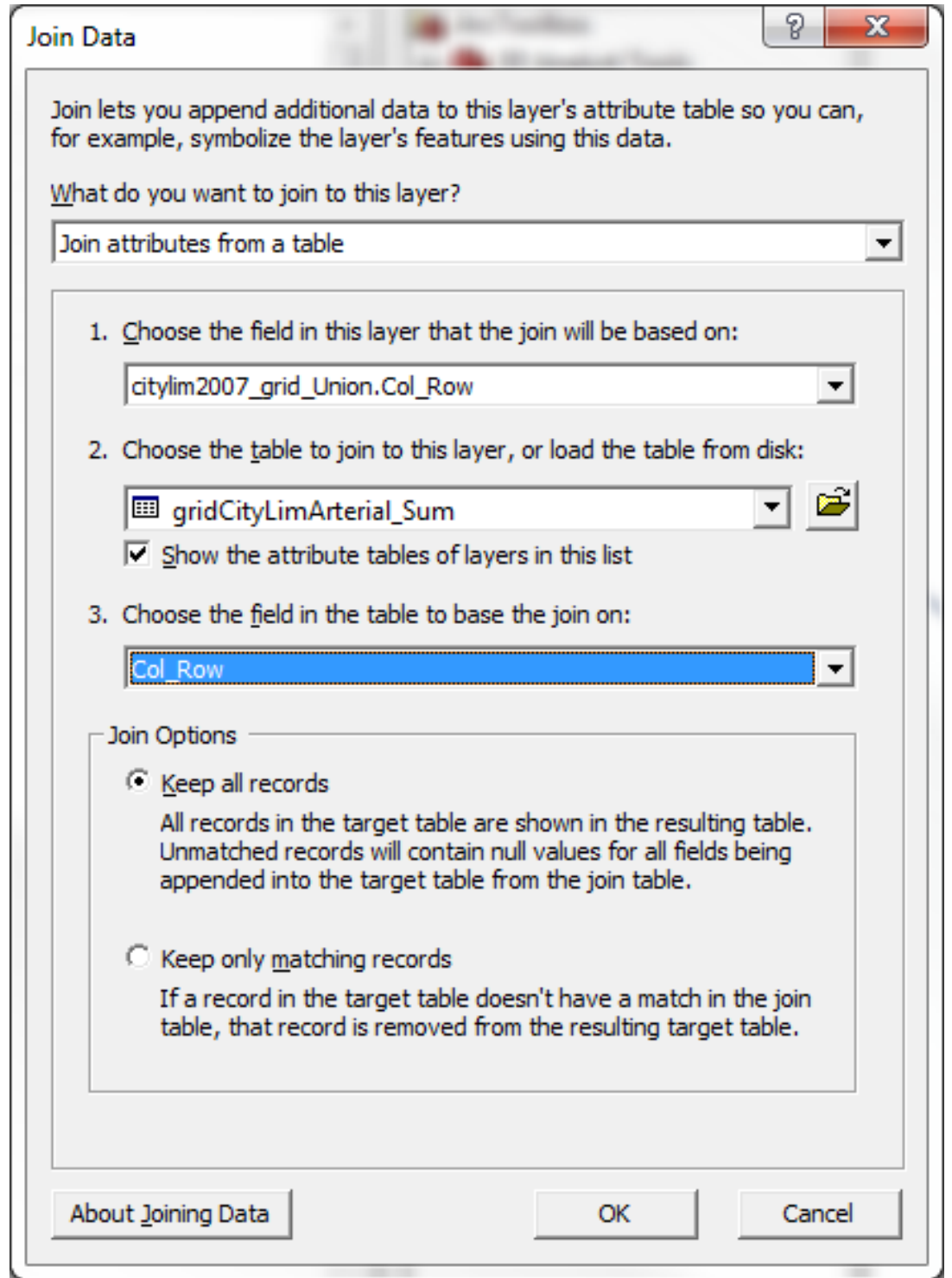

Hit OK-if prompted hit yes

The summary value should now appear as a column in the polygon attribute table.. 


\section{APPENDIX D: Apportion polygon data to a different polygon spatial}

area in ArcGIS 9.3.1

Example for employment by TAZ being put into uniform grid layer

1. Create smaller TAZ/Employment areas contained within each grid cell

a. Select the "ArcToolbox from the top (it's a red icon, looks like a toolbox) Analysis Tools -> Overlay - > Union

For Input, select the "Employment" and the shapefile for the layer with the polygons data are going into.

Make sure the Output file is named what you want it to be

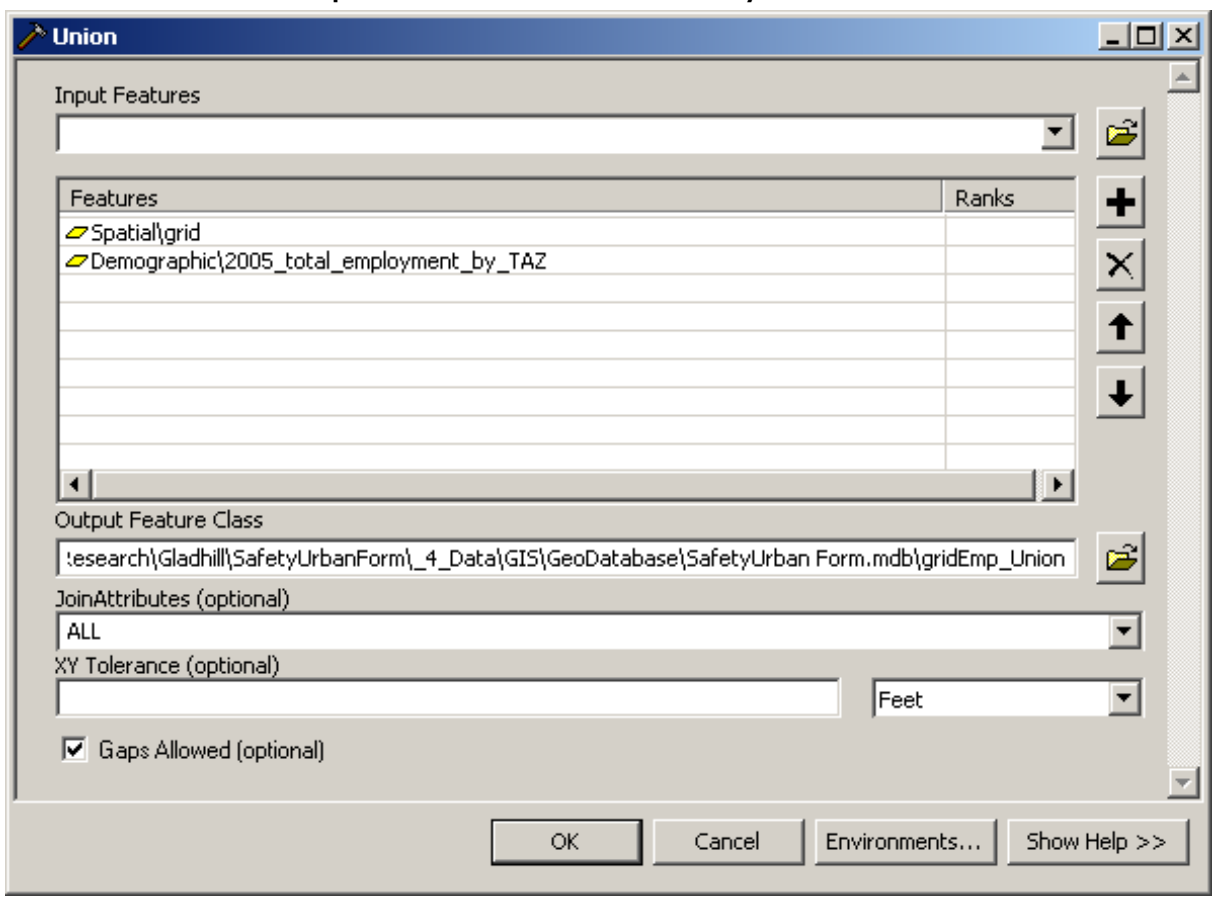

Hit OK-It will take a bit, but the new shapefile should be automatically added to the map. 
2. Clean-up output layer and perform calculations

a. Right click output layer and select "Open Attribute Table"

b. Delete all unnecessary columns by selecting from the top of each column).

c. While still in the Attributes window, select "Options" (at the bottom of the dialogue box), then "Add Field" (to be the new "area" of the smaller TAZ/Employment areas)

d. Enter information as show below, and hit OK:

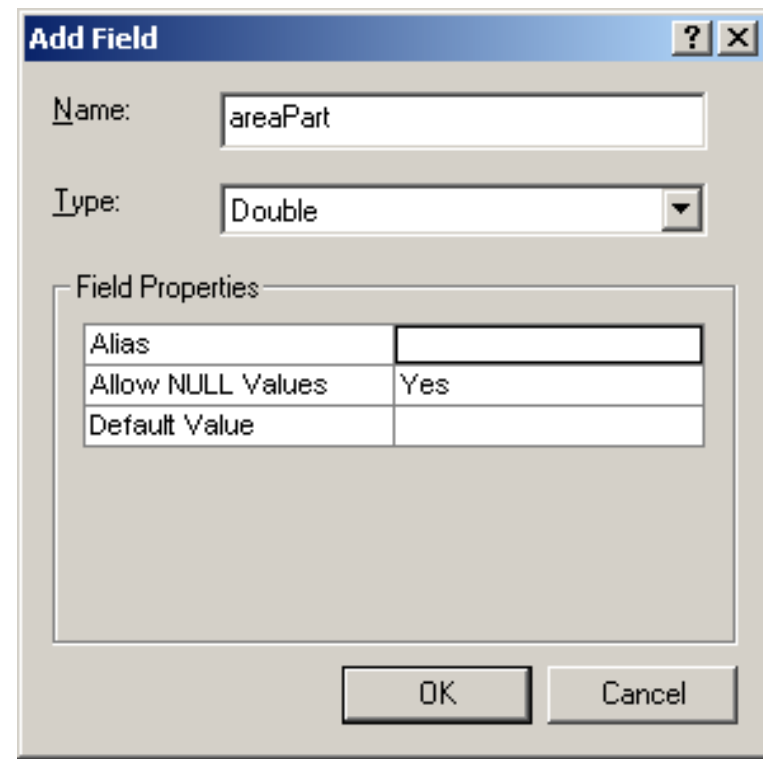


e. To populate the new "AreaPart" Right click the "AreaPart" column, select "Calculate Geometry" hit "yes" and enter information as show below:

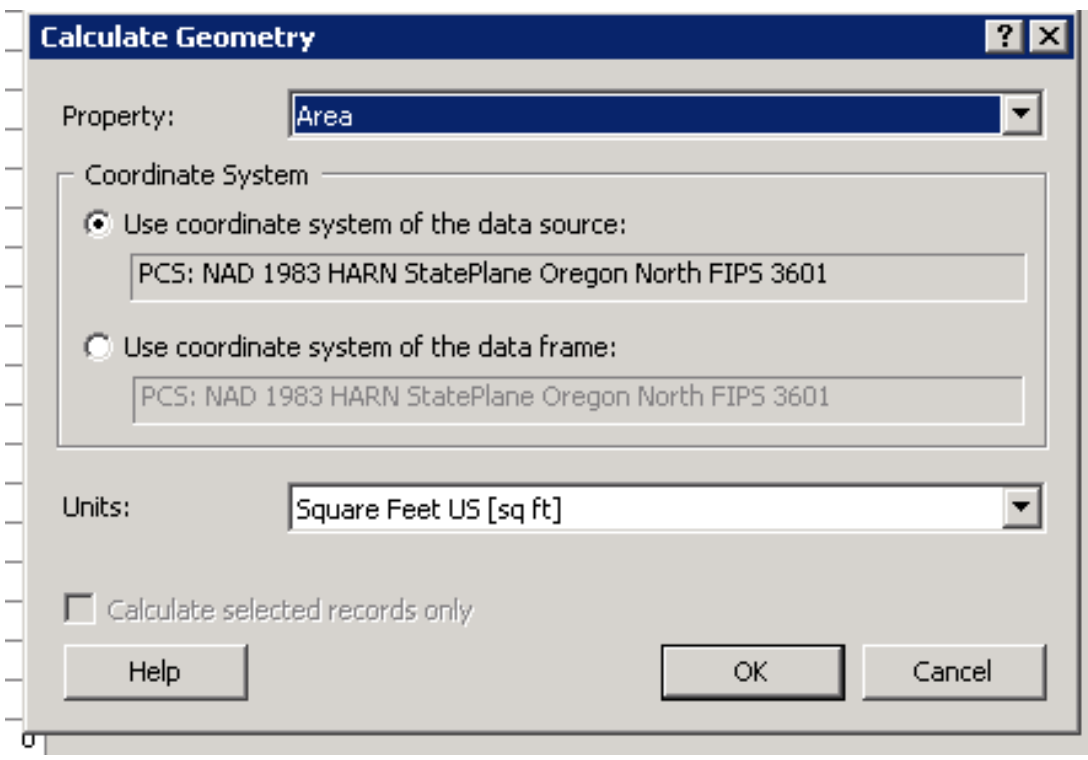

Much of the information above should be there (if not all)-hit OK.

f. Create a new field-this will be to calculate the percentage of the total TAZ/Employment area that is in the grid cell. Field information is as below:

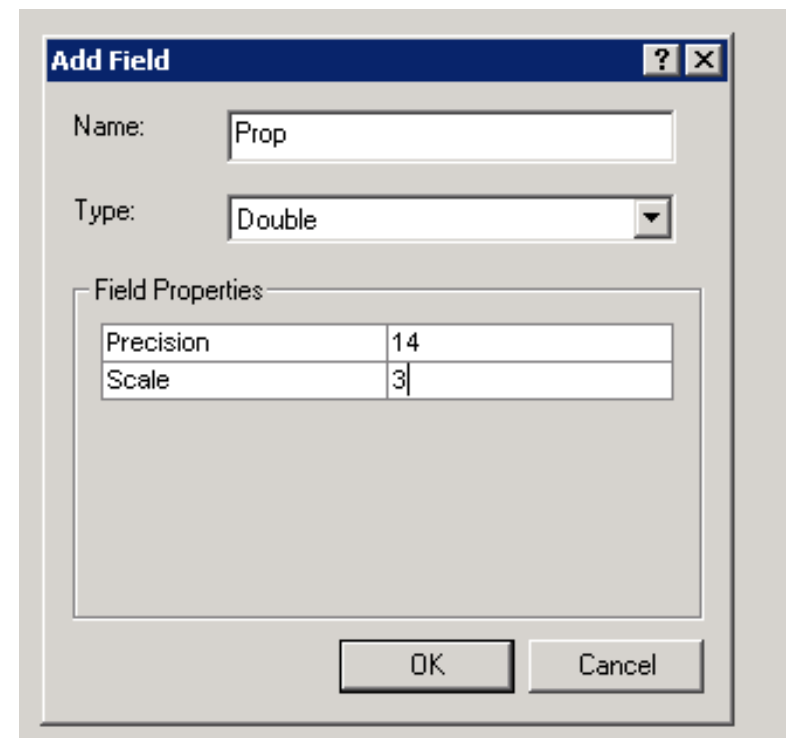

Page 95 
g. Select the new area field, Right-Click and select "Field Calculator" and "Yes". Using the Mouse to select the Values (sometimes if you use keyboard it doesn't work right), select AreaPart / Area and hit OK.

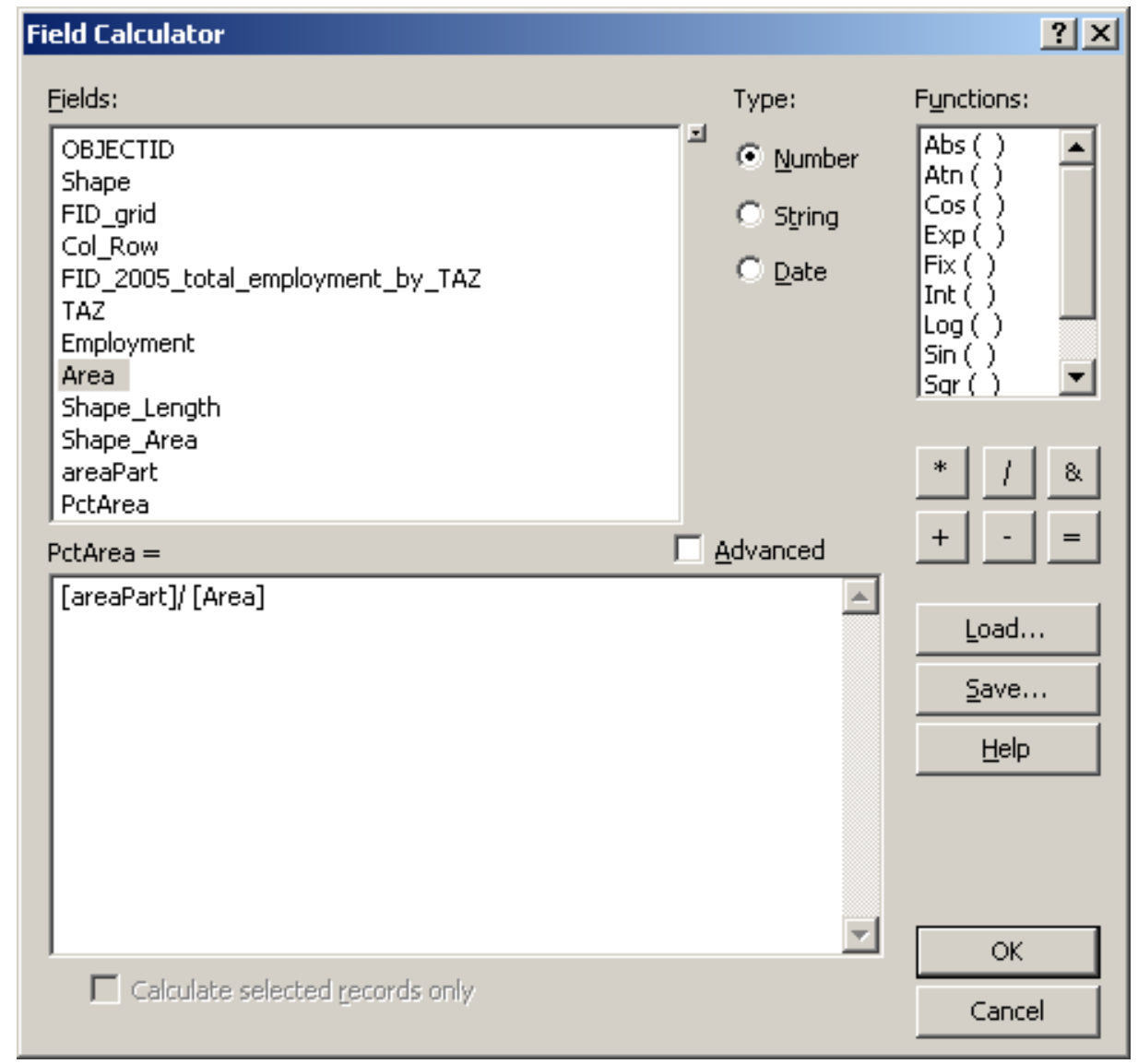

If there is a prompt (from an error) hit "yes"-this "error" is because some cells have zero "Area" because the TAZ/Employment does not cover the entire grid. 
h. Create a new field for the Proportion of Employment in the smaller area by selecting "options", "add new field named something like "PortionEmp".

i. Select the "PortionEmp" field, Right-Click and select "Field Calculator" and "Yes".

Select the values: [PctArea] * [Employment] Hit OK when done, and yes if there is a prompt.

j. Format the EmpGrid_Union and label the "PortionEmp" field by following steps from above and shown below:

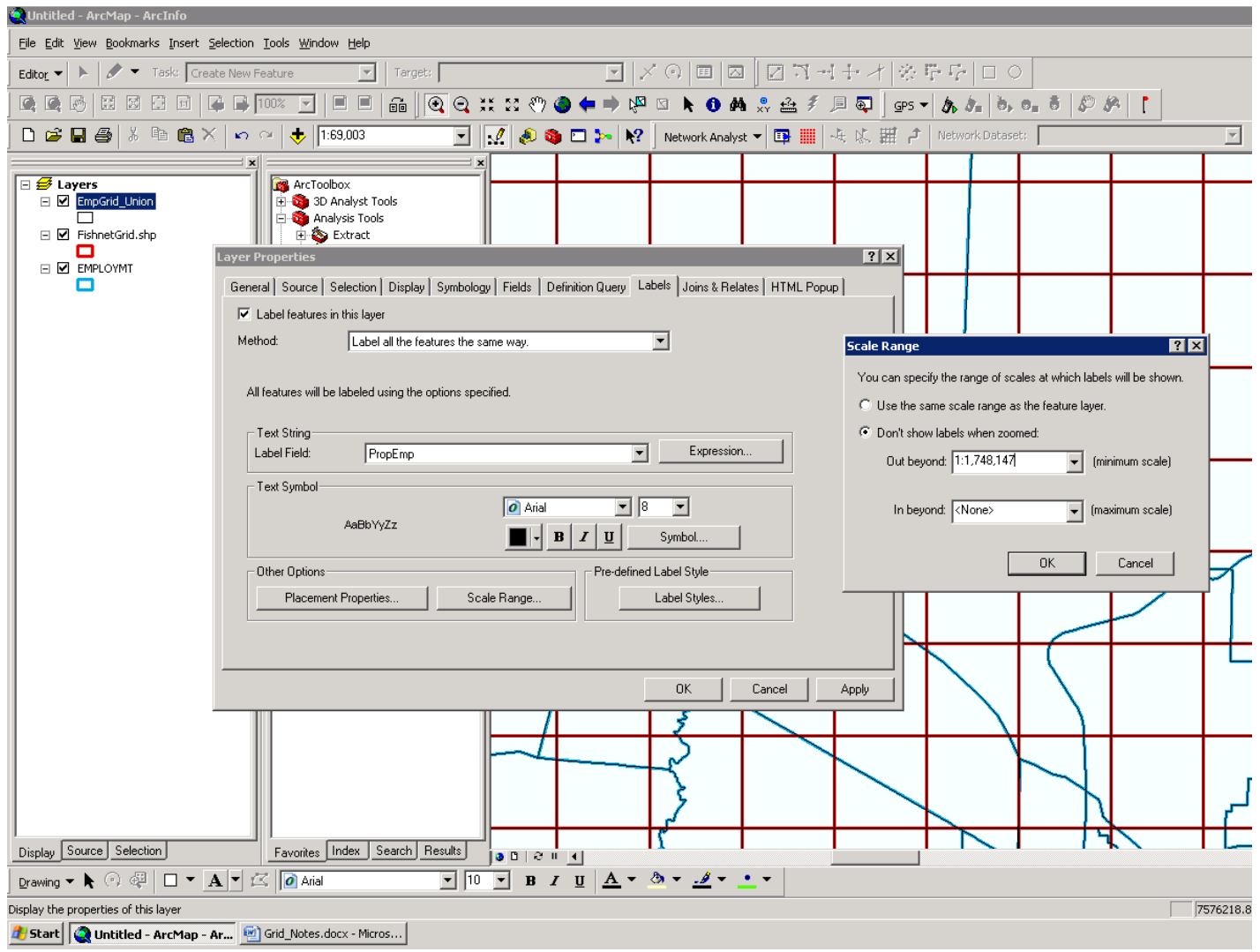

k. Hit Apply and OK 
3. Sum the Smaller TAZ/Employment totals that fall within the grid cells

a. From the ArcToolbox,

'Analysis Tools -> Statistics ->Summary Statistics

For the Statistics field, select the 'PortionEmp'-this is the proportion of employment within the smaller TAZ/Employment areas (that fall within a given grid). They will need to be summed up within each grid (so choose 'SUM' for the statistic type in the drop down, as shown above).

For the case field, you will have to select, the 'Col_Row' field-this is because you want to sum by each grid (denoted by the Col_Row coordinate).

Hit OK, and Close-it will look like nothing happened...that's OK. 


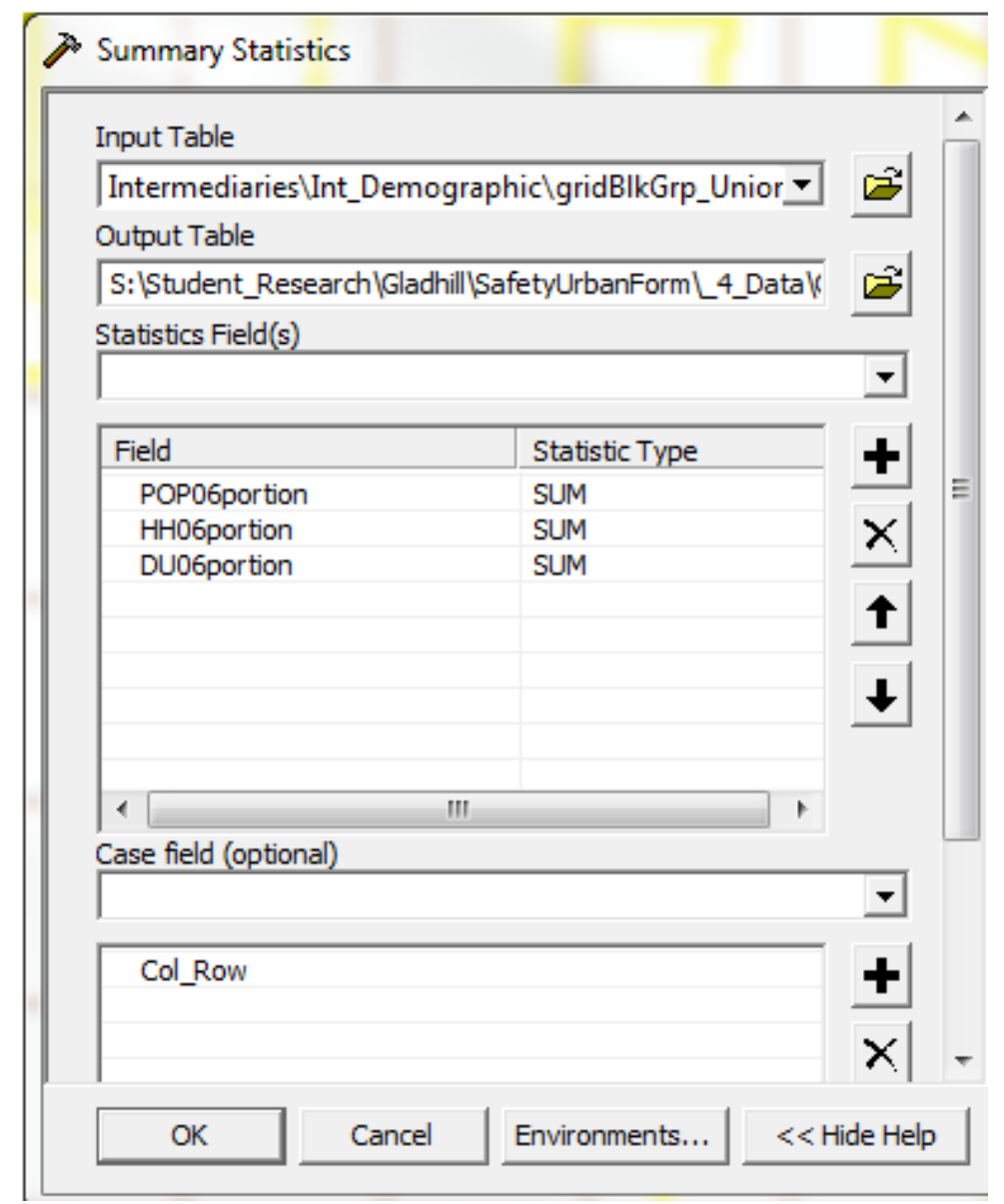

b. In the Table of Contents window, select the 'grid' layer by Right-click, and select: Join and Relates -> Join... 


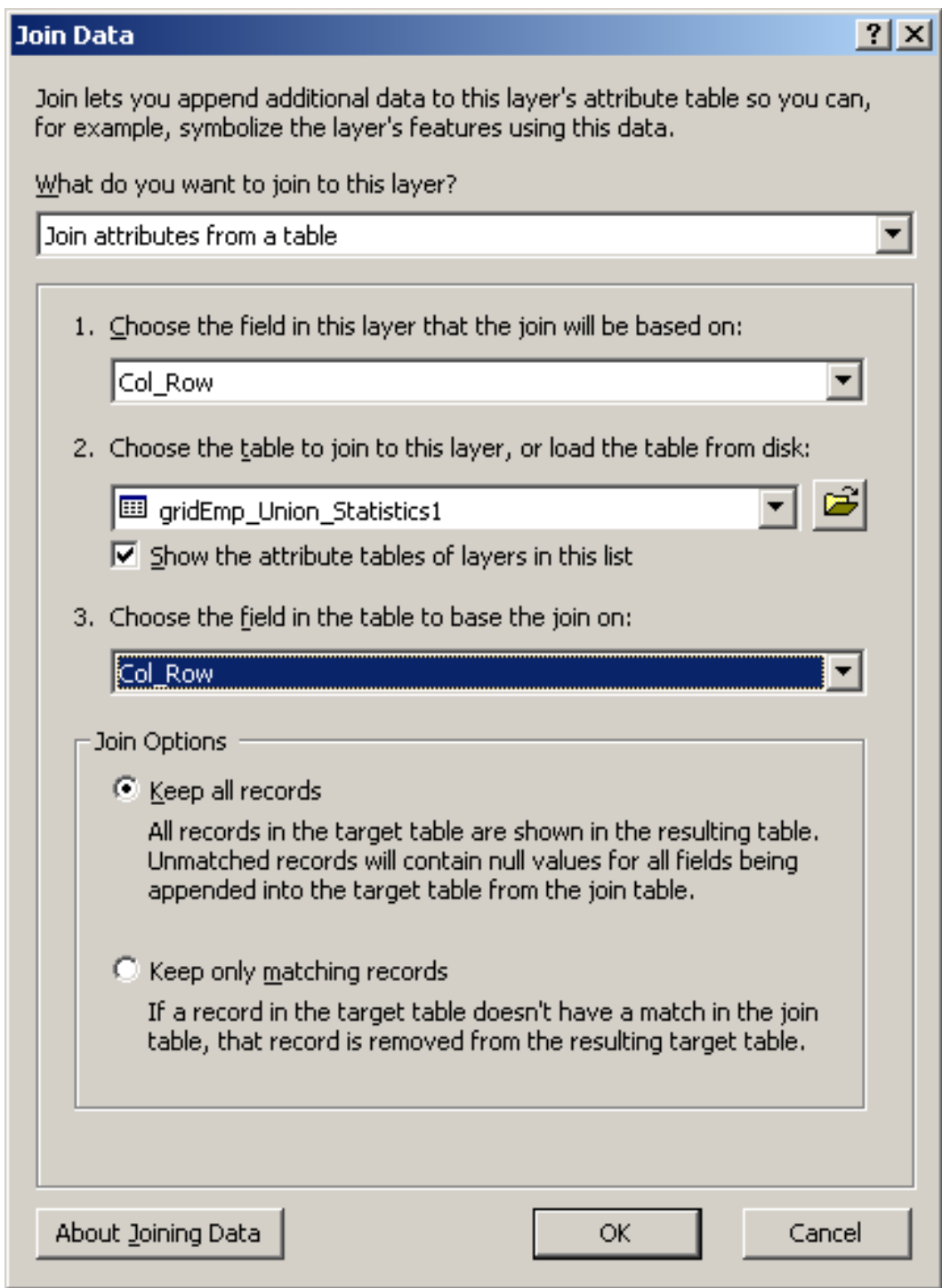

Hit OK—if prompted hit yes

Note: gridEmp_Union_Statistics1 can be found under Source tab, 
APPENDIX E: Pearson's Correlation on Factors - Figure E-1 Correlation for Exposure Factors

\begin{tabular}{|c|c|c|c|c|c|c|c|c|c|c|}
\hline & $\begin{array}{l}\text { 咅 } \\
\text { 咅 } \\
\text { 总 }\end{array}$ & $\begin{array}{l}\overleftarrow{U} \\
\stackrel{0}{0} \\
\frac{d}{\sigma}\end{array}$ & 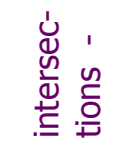 & 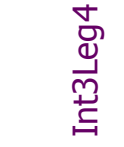 & 炰 & 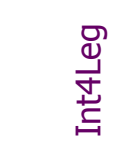 & 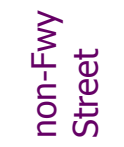 & $\begin{array}{l}\frac{\overline{0}}{\frac{0}{\bar{N}}} \\
\frac{\pi}{\frac{1}{4}}\end{array}$ & $\begin{array}{l}\frac{\pi}{\Delta} \\
\frac{\pi}{2}\end{array}$ & 음 $\frac{\tilde{N}}{\tilde{N}}$ \\
\hline 4legPct & 0.619 & & & & & & & & & \\
\hline intersections & 0.666 & 0.501 & & & & & & & & \\
\hline Int3Leg4 & 0.699 & 0.604 & 0.944 & & & & & & & \\
\hline Int3Leg & 0.385 & -0.057 & 0.603 & 0.627 & & & & & & \\
\hline Int4Leg & 0.644 & 0.825 & 0.784 & 0.842 & 0.112 & & & & & \\
\hline nonFwyStreet & 0.794 & 0.660 & 0.889 & 0.920 & 0.541 & 0.805 & & & & \\
\hline gridStreet & 0.754 & 0.641 & 0.908 & 0.924 & 0.527 & 0.812 & 0.970 & & & \\
\hline gridArterial & 0.106 & 0.190 & 0.416 & 0.388 & 0.143 & 0.361 & 0.326 & & & \\
\hline gridMajArt & 0.006 & 0.130 & 0.322 & 0.287 & 0.034 & 0.301 & 0.208 & 0.889 & & \\
\hline blockSize & 0.538 & 0.524 & 0.905 & 0.886 & 0.444 & 0.802 & 0.797 & 0.679 & 0.620 & \\
\hline transitStops & 0.265 & 0.296 & 0.367 & 0.396 & 0.144 & 0.407 & 0.386 & 0.240 & 0.187 & 0.375 \\
\hline busStops & 0.266 & 0.296 & 0.367 & 0.396 & 0.144 & 0.407 & 0.386 & 0.237 & 0.184 & 0.374 \\
\hline transitRoute & 0.170 & 0.246 & 0.348 & 0.359 & 0.111 & 0.372 & 0.332 & 0.426 & 0.388 & 0.434 \\
\hline busRoute & 0.172 & 0.248 & 0.347 & 0.359 & 0.111 & 0.372 & 0.331 & 0.414 & 0.374 & 0.429 \\
\hline ons & 0.149 & 0.198 & 0.236 & 0.265 & 0.054 & 0.294 & 0.229 & 0.211 & 0.217 & 0.278 \\
\hline offs & 0.151 & 0.202 & 0.244 & 0.273 & 0.052 & 0.307 & 0.233 & 0.215 & 0.224 & 0.288 \\
\hline onOff & 0.151 & 0.201 & 0.241 & 0.270 & 0.053 & 0.302 & 0.232 & 0.214 & 0.222 & 0.284 \\
\hline estLoad & 0.231 & 0.282 & 0.341 & 0.381 & 0.113 & 0.401 & 0.347 & 0.267 & 0.252 & 0.387 \\
\hline VMT & 0.093 & 0.156 & 0.363 & 0.325 & 0.118 & 0.299 & 0.238 & 0.841 & 0.844 & 0.600 \\
\hline VCdist & 0.239 & 0.293 & 0.478 & 0.468 & 0.199 & 0.430 & 0.423 & 0.824 & 0.729 & 0.667 \\
\hline avgVC & 0.111 & 0.128 & 0.172 & 0.169 & 0.095 & 0.146 & 0.177 & 0.318 & 0.261 & 0.232 \\
\hline AvgFreeSpeed & -0.443 & -0.349 & -0.339 & -0.388 & -0.256 & -0.338 & -0.446 & 0.213 & 0.311 & -0.189 \\
\hline schools & 0.204 & 0.193 & 0.236 & 0.260 & 0.137 & 0.239 & 0.244 & 0.033 & 0.006 & 0.207 \\
\hline Pop06 & 0.629 & 0.519 & 0.569 & 0.618 & 0.431 & 0.516 & 0.669 & 0.066 & -0.041 & 0.445 \\
\hline HH06 & 0.563 & 0.535 & 0.560 & 0.623 & 0.340 & 0.577 & 0.641 & 0.144 & 0.052 & 0.488 \\
\hline DU06 & 0.555 & 0.533 & 0.562 & 0.624 & 0.338 & 0.580 & 0.638 & 0.153 & 0.064 & 0.495 \\
\hline employment & 0.117 & 0.251 & 0.244 & 0.286 & -0.032 & 0.381 & 0.223 & 0.217 & 0.248 & 0.315 \\
\hline
\end{tabular}




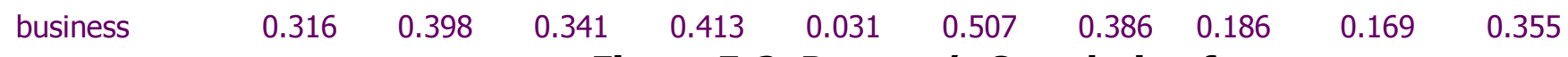

Figure E-2, Pearson's Correlation for

Transit accessibility, Exposure, Demographic, and Origin Destination Factors

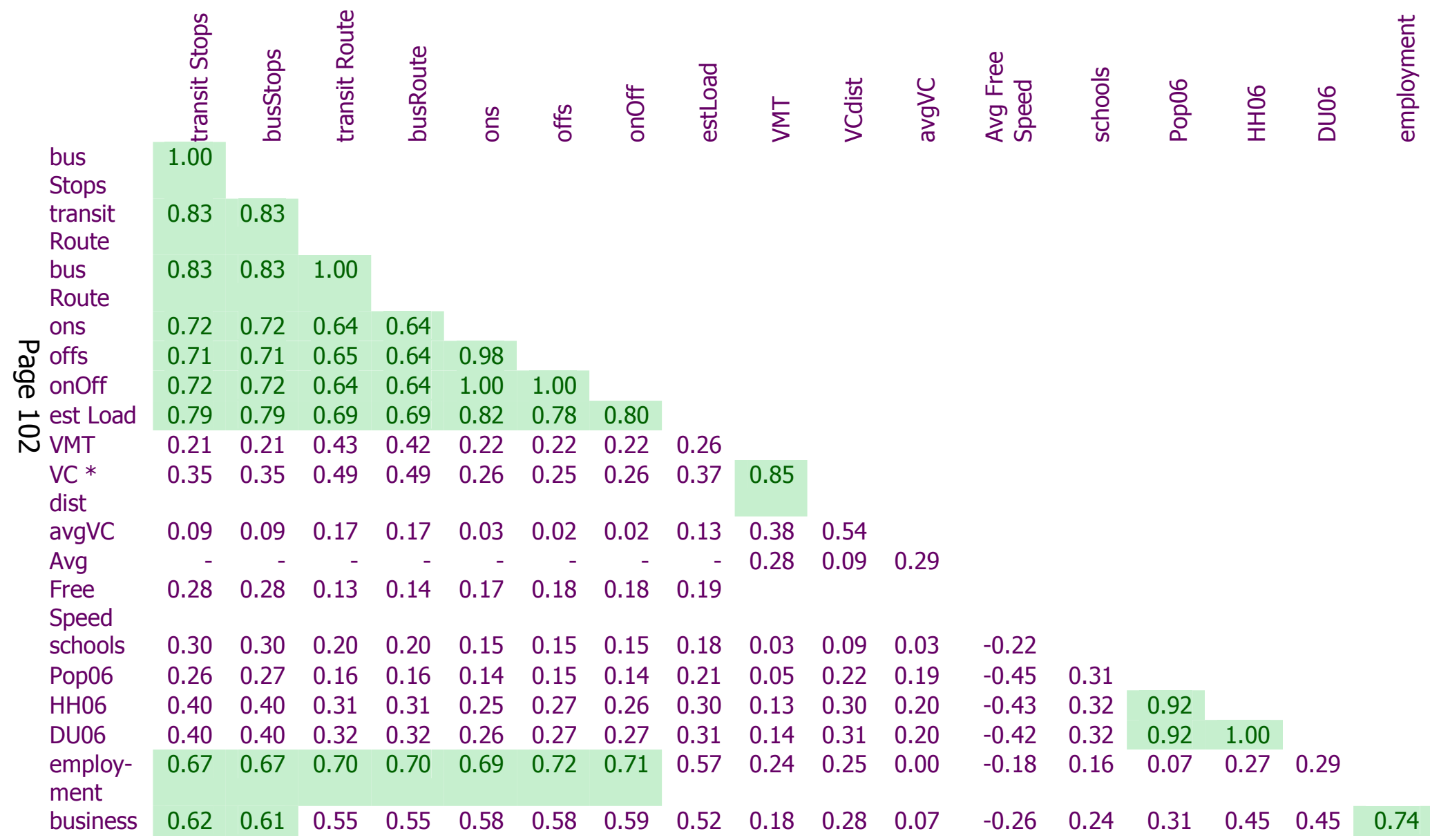

